\title{
State-of-the-Art of Optimal Active and Reactive Power Flow: A Comprehensive Review from Various Standpoints
}

\author{
Ehsan Naderi ${ }^{1, *(\mathbb{C}}$, Hossein Narimani ${ }^{2}$, Mahdi Pourakbari-Kasmaei ${ }^{3}{ }^{(}$, Fernando V. Cerna ${ }^{4}(\mathbb{D}$, \\ Mousa Marzband ${ }^{5}$ (D) and Matti Lehtonen ${ }^{3}$ (D) \\ 1 School of Electrical, Computer, and Biomedical Engineering, Southern Illinois University, \\ Carbondale, IL 62901, USA \\ 2 Department of Electrical Engineering, Kermanshah Branch, Islamic Azad University, \\ Kermanshah 67189-97551, Iran; Hosein.Narimani83@gmail.com \\ 3 Department of Electrical Engineering and Automation, Aalto University, Maarintie 8, 02150 Espoo, Finland; \\ Mahdi.Pourakbari@aalto.fi (M.P.-K.); Matti.Lehtonen@aalto.fi (M.L.) \\ 4 Department of Electrical Engineering, Federal University of Roraima, Boa Vista 69310-000, Brazil; \\ Fernando.Cerna@ufrr.br \\ 5 Department of Mathematics, Physics, and Electrical Engineering, Faculty of Engineering and Environment, \\ Northumbria University, Newcastle upon Tyne NE1 8ST, UK; Mousa.Marzband@northumbria.ac.uk \\ * Correspondence: Ehsan.Naderi@siu.edu
}

Citation: Naderi, E.; Narimani, H.; Pourakbari-Kasmaei, M.; Cerna, F.V.; Marzband, M.; Lehtonen, M State-of-the-Art of Optimal Active and Reactive Power Flow: A Comprehensive Review from Various Standpoints. Processes 2021, 9, 1319 https://doi.org/10.3390/pr9081319

Academic Editor: Ambra Giovannelli

Received: 17 May 2021

Accepted: 26 July 2021

Published: 29 July 2021

Publisher's Note: MDPI stays neutral with regard to jurisdictional claims in published maps and institutional affiliations.

Copyright: (c) 2021 by the authors. Licensee MDPI, Basel, Switzerland. This article is an open access article distributed under the terms and conditions of the Creative Commons Attribution (CC BY) license (https:// creativecommons.org/licenses/by/ $4.0 /$ )

\begin{abstract}
Optimal power flow (OPF), a mathematical programming problem extending power flow relationships, is one of the essential tools in the operation and control of power grids. To name but a few, the primary goals of OPF are to meet system demand at minimum production cost, minimum emission, and minimum voltage deviation. Being at the heart of power system problems for half a century, the OPF can be split into two significant categories, namely optimal active power flow $(\mathrm{OAPF})$ and optimal reactive power flow (ORPF). The OPF is spontaneously a complicated non-linear and non-convex problem; however, it becomes more complex by considering different constraints and restrictions having to do with real power grids. Furthermore, power system operators in the modern-day power networks implement new limitations to the problem. Consequently, the OPF problem becomes more and more complex which can exacerbate the situation from mathematical and computational standpoints. Thus, it is crucially important to decipher the most appropriate methods to solve different types of OPF problems. Although a copious number of mathematical-based methods have been employed to handle the problem over the years, there exist some counterpoints, which prevent them from being a universal solver for different versions of the OPF problem. To address such issues, innovative alternatives, namely heuristic algorithms, have been introduced by many researchers. Inasmuch as these state-of-the-art algorithms show a significant degree of convenience in dealing with a variety of optimization problems irrespective of their complexities, they have been under the spotlight for more than a decade. This paper provides an extensive review of the latest applications of heuristic-based optimization algorithms so as to solve different versions of the OPF problem. In addition, a comprehensive review of the available methods from various dimensions is presented. Reviewing about 200 works is the most significant characteristic of this paper that adds significant value to its exhaustiveness.
\end{abstract}

Keywords: constraint optimization; cybersecurity; heuristic algorithms; meta-heuristic algorithms; optimization methods; optimal power flow (OPF); optimal reactive power dispatch (ORPD)

\section{Introduction}

\subsection{Background and Definitions}

Over the years, optimal power flow (OPF) problem has been the backbone of the operation and control of any power network since the early 1960s. However, nowadays, it is widely used to dispatch the available generation of power plants so that a particular 
objective function or a set of objectives are optimized simultaneously [1]. In addition, the OPF problem can fully represent and handle the network equations and nodal power balance. It also maintains acceptable boundaries on bus voltage, power flow of branches, and the output power of generators. It should be noted that conventional OPF formulation entails minimizing total operation cost and network losses. However, solving the OPF problem only from an economic stance cannot take care of other significant, inevitable, and unbridled issues connected with modern-day power networks. As an illustration of this, in the past few years, the US environmental protection agency coerced the generation power factories to serve the demand not only at minimum cost but also with the minimum amount of contaminations. In this regard, to address the aforementioned issues, the OPF problem has to be considered as a Multi-Objective Optimization Problem (MOOP) capable of satisfying all expectations at the same time. To this end, all of the other important and well-known objective functions in this avenue of research are classified and scrutinized in detail in this paper. Moreover, optimal reactive power dispatch (ORPD), another type of the OPF problem, is also an inevitable and crucially important problem to ensure the secure operation of power networks due to the fact that controlling the voltage profile is highly related to regulating reactive power flow, which is the cornerstone of ORPD study.

Research on solution techniques for both the OPF and the ORPD problems has been active since Carpentier first introduced it in 1962 [2]. In this connection, quite a few mathematical programming methods [3] such as the Newton method [2], Quadratic Programming (QP) [4], Linear Programming (LP) [5], Non-Linear Programming (NLP) [6], and Interior Point (IP) methods [7] have been utilized to handle the problems, which are quite difficult optimization problems in realistic and practical power grids. However, mathematical approaches have some counterpoints, such as suitability to specific OPF problems and applicability to only continuous problems, to name just two $[8,9]$. Furthermore, the mathematical optimization methods that use derivatives and gradients can give the optimal solution only when the objective function is completely continuous and, as a result, a differentiable one. As an illustration, the gradient and Newton methods suffer from counterpoints in satisfying inequality constraints. Moreover, the input-output function is written as a set of linear functions when LP methods are applied to solve the problem, which may eventuate in loss of preciseness. Nevertheless, both OPF and ORPD problems are inherently non-convex and non-smooth due to their variables and characteristics of the generator's cost function. In this regard, all aforementioned drawbacks can be completely covered if apt evolutionary methods are implemented to solve the problem.

Evolutionary Algorithms (EAs) have become popular tools to cope with a variety of complicated optimization problems. EAs solve the problems by generating a random, initial population in which each member is assessed by the numeral value of the objective function. Therefore, they can handle every kind of optimization problem irrespective of their qualitative discrepancies [10,11]. In general, the best methods for solving different models of the OPF problem can be categorized as (a) mathematical-based methods and (b) evolutionary-based algorithms, the detailed classifications of which are elaborated upon in Figure 1. In this connection, it should be noted that this paper is focused on evolutionary-based methods for solving the problem from different standpoints (e.g., algorithms, objectives, and test systems, to name just a handful). 


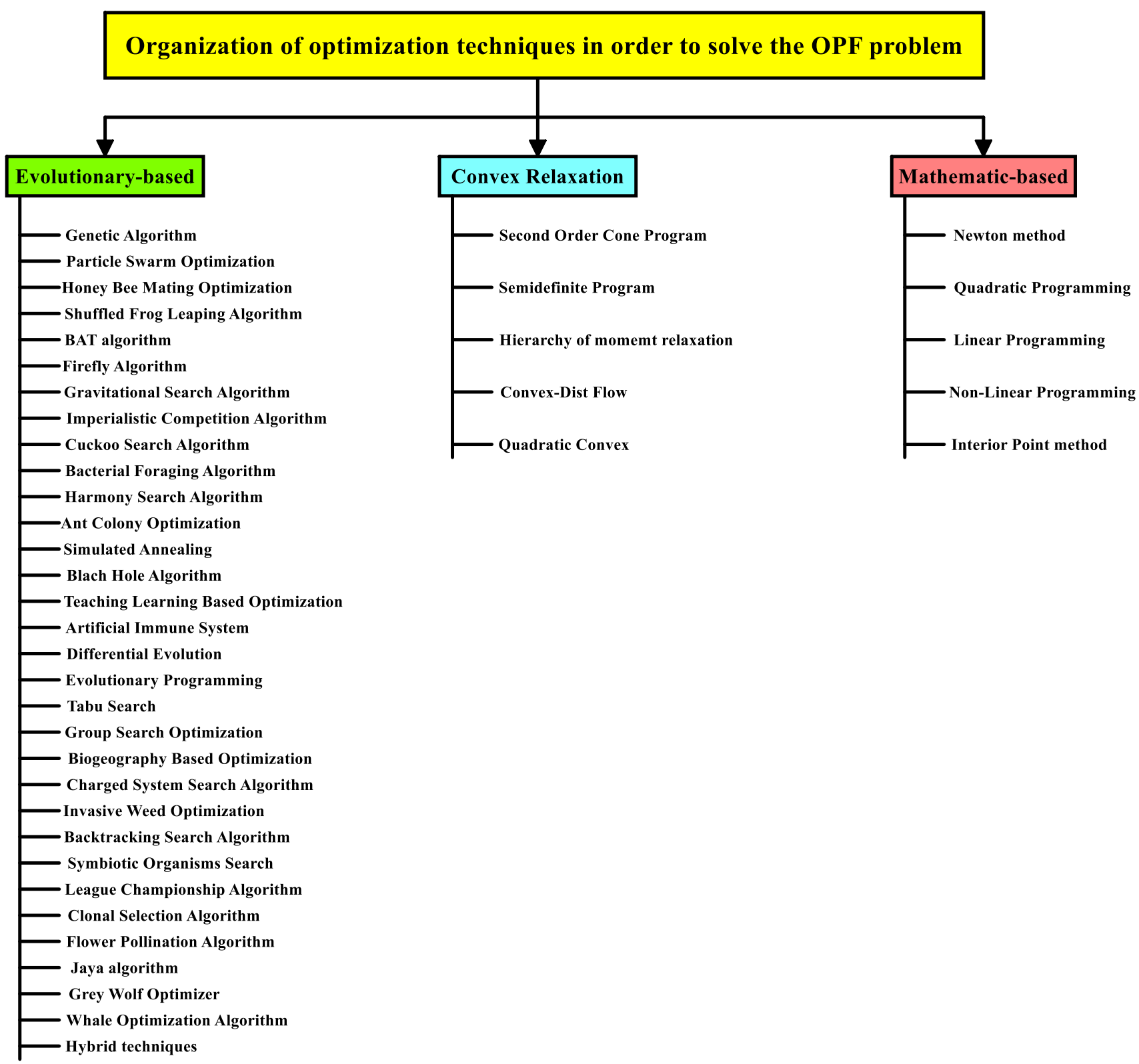

Figure 1. Classification of the optimal power flow solution methodologies.

As pointed out earlier, the primary objective of OPF problems is minimizing the overall operation cost of the system. Nevertheless, there are other fitness functions that can be considered rather than or beside the cost function, such as minimizing the active power losses, bus voltage deviation, and emission, etc. [2]. In addition, the static OPF usually results in undesirable and imprecise costs in a multi-period context; thus, to prevent this issue, the OPF must be solved as dynamic optimal power flow (DOPF) that extends over multiple time slots.

The basic OPF problem can be extended simply to solve other optimization problems in the power industry. In this connection, all applications of the OPF and the ORPD problems are demonstrated in Figure 2. 


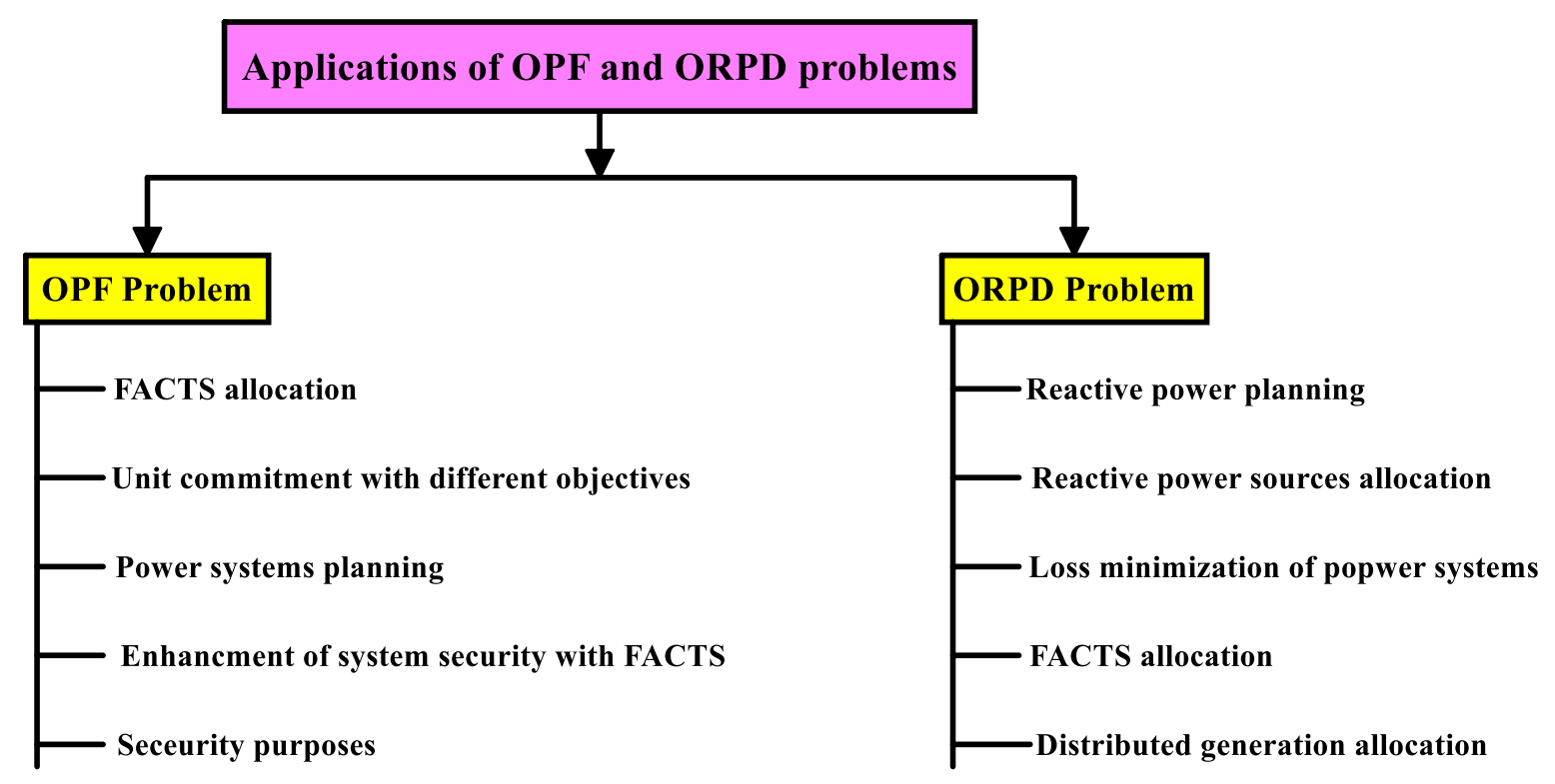

Figure 2. Important applications of OPF and ORPD problems in power systems.

\subsection{Motivation}

The annually constant influx of new, innovative optimization methods entail heuristic and meta-heuristic algorithms for solving various versions of the OPF problem, which motivates us to feel that a comprehensive job in reviewing these evolutionary algorithms implemented to handle the problem is inevitable so much so that it is becoming a broad avenue of research. Moreover, no complete and comprehensive literature review has been introduced thus far on the problem to provide researchers with a united reference. To this end, it is necessary to classify all employed EAs applied to the OPF problem from different stances, which can be considered as the main contribution of this study.

\subsection{Scope}

It is notable that there are quite a few benchmarks to opt for references so as to prepare for reviewing papers. For instance, a paper can be cited according to a variety of criteria such as its topic, objectives, contributions, publication year, and place of publication, to name but a few. This paper reviews and compares all the available solving approaches from various aspects as well as their performance on minimizing the cost objective function. With this in mind, this paper develops a comprehensive review of the OPF problem oriented to the subject, objective functions, constraints and limitations, solver and algorithms, test systems, obtained values of the objective function, integration of renewable energy resources and energy storage systems, and finally contributions.

\subsection{Organization of the Paper}

The rest of the paper is elaborated upon hereunder.

Section 2 presents the OPF problem formulation in detail that entails introducing the problem, objective functions, and all constraints and limitations. In Section 3, all heuristic and meta-heuristic approaches applied to different versions of the OPF problem are reviewed in detail. Elaborating the recent researches from various aspects is scrutinized in Section 4, and conclusions of the paper are briefly summarized in Section 5.

\section{Thorough Representation of OPF and ORPD}

This section initially introduces AC Power Flow (ACPF), and then the mathematical modeling of the OPF problem is scrutinized. The ACPF is according to complex quantities such as voltage, current, apparent power, and admittance, to name just a handful. The aforementioned characteristics are inextricably interwoven based on (1) Krichhoff's Current 
Law (KCL), (2) Ohm's law, (3) the definition of apparent power, and finally, (4) and (5), the backbone of AC power flow [12]. They are elaborated as follows:

$$
\begin{gathered}
I_{i}^{G}-I_{i}^{D}=\sum_{j \in \Omega_{i}} I_{i j} \\
I_{i j}=Y_{i j} \times\left(V_{i}-V_{j}\right) \\
S_{i j}=V_{i} \times I_{i j}^{*} \\
S_{i}^{G}-S_{i}^{D}=\sum_{j \in \Omega_{i}} S_{i j} ; \forall i \\
S_{i j}=Y_{i j}^{*} \times V_{i} \times V_{i}^{*}-Y_{i j}^{*} \times V_{i} \times V_{j}^{*} ; \forall j
\end{gathered}
$$

where $\Omega_{i}$ is the set of buses directly connected to bus $i$.

In this regard, (1)-(5) should be solved subject to many constraints and restrictions on the operational side, the most conspicuous of which are elaborated upon hereunder.

- Capabilities of generation units: The active and reactive power production of all generators are bounded between the minimum and maximum boundaries.

- Boundaries for flowing of line: All AC power lines have thermal restrictions in VA unit in order to prevent sagging and unexpected activation of protection equipment $[1,3]$.

- Boundaries for voltage magnitude of buses: All voltage magnitudes in power networks should not change exceptionally and must be close to their nominal values.

- Phase angle difference: A difference of phase angles should be small, even less than ten degrees.

- Other constraints, such as limits of active power/apparent power (i.e., MW/MVA) flowing between different areas, are not considered most of the time due to the nature of available test systems, which consider only one region.

The OPF problem is principally an ACPF with considering several extra constraints and limitations, which is elaborated upon in detail in this section. Moreover, the OPF problem can be split into two major sub-problems, OPF and ORPD, both of which are completely covered in this section. It should be noted that different objective functions can be taken into account in both OPF and ORPD problems; Figure 3 portrays an outline and classification of the objective functions.

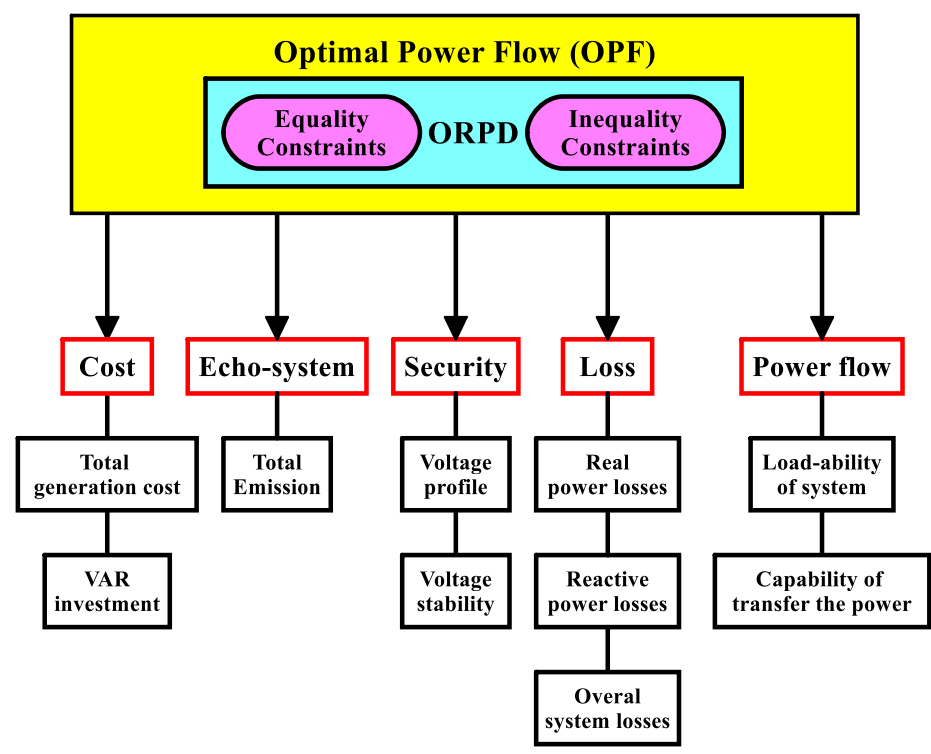

Figure 3. Overview of important objective functions in both OPF and ORPD problems. 


\subsection{Single-Objective Approach}

This version of the OPF problem is formulated to minimize only one fitness function, which almost always is total generation cost with and without considering practical constraints, different versions of emission function and function of power losses, voltage deviation and voltage stability index so as to enhance the stability of power grids. On the other hand, for solving the single-objective version of the ORPD problem, the favorable objective functions entail power losses and VAR cost, voltage deviation, and voltage stability index, to name but three.

\subsubsection{Optimal Power Flow}

In the realm of OPF study, the generation cost function accompanied by control variables can be mathematically expressed as (6)-(12) where $X$ indicates the vector of control variables. It should be noted that the objective function is minimized subject to satisfying (49) and (50) and also (54)-(61) as equality and inequality constraints, respectively (see Section 2.1.4).

$$
\begin{gathered}
C_{Q}(X)=\min \sum_{i=1}^{N_{G}}\left(a_{i} P_{g i}^{2}+b_{i} P_{g i}+c_{i}\right) \\
X=\left[P_{g}, V_{g}, T A P, Q_{c}\right]_{1 \times n} \\
P_{g}=\left[P_{g 1}, P_{g 2}, \ldots, P_{g\left(N_{G}-1\right)}\right]_{1 \times\left(N_{G}-1\right)} \\
V_{g}=\left[V_{g 1}, V_{g 2}, \ldots, V_{g N_{G}}\right]_{1 \times N_{G}} \\
T A P=\left[T A P_{1}, T A P_{2}, \ldots, T A P_{N_{T}}\right]_{1 \times N_{T}} \\
Q_{c}=\left[Q_{c 1}, Q_{c 2}, \ldots, Q_{c N_{c}}\right]_{1 \times N_{c}} \\
n=\left(N_{T}+N_{c}+N_{G}+\left(N_{G}-1\right)\right)
\end{gathered}
$$

In this connection, the vectors of control variables as well as state variables are elaborated upon hereunder.

Control variables entail output active power at PV buses apart from the slack bus, voltage amplitude at PV buses, settings of transformers' tap, and shunt VAR compensators.

State variables involve output active power at the slack bus, voltage amplitude at PQ buses, output reactive power of all generators, and transmission line flow.

For having a holistic view, all of the variables in the area of solving the OPF problem can be outlined in Table 1, which is based on types of variables.

Table 1. Classification of OPF and ORPD variables.

\begin{tabular}{cc}
\hline Variables & Variable Types \\
\hline Control variables & \\
Active power generation & $\mathrm{C}^{1}$ \\
Reactive power generation & $\mathrm{C}$ \\
Voltage amplitude of buses & $\mathrm{C}$ \\
Voltage control setting of generators & $\mathrm{C}$ \\
Position of the transformer tap & $\mathrm{C} / \mathrm{D}^{2}$ \\
Position of the transformer phase shifters & $\mathrm{C} / \mathrm{D}$ \\
Lon $/ \mathrm{D}$ \\
Load shedding & $\mathrm{C} / \mathrm{D}$ \\
Statues of the switched shunt reactive components & $\mathrm{B}{ }^{3}$ \\
Switching the transmission lines & $\mathrm{B}$ \\
Start-up generators & $\mathrm{B}$ \\
\hline
\end{tabular}


Table 1. Cont.

\begin{tabular}{|c|c|}
\hline Variables & Variable Types \\
\hline \multicolumn{2}{|l|}{ State variables } \\
\hline $\begin{array}{l}\text { Active power of slack bus except for PV buses, which are } \\
\text { decision variables }\end{array}$ & $\mathrm{C}$ \\
\hline Output reactive power of generators & $\mathrm{C}$ \\
\hline Amplitude of bus voltages & $\mathrm{C}$ \\
\hline Phase angle of bus voltage except for the slack bus & $\mathrm{C}$ \\
\hline Real and imaginary parts of bus voltage & $\mathrm{C}$ \\
\hline Power flow of system & $\mathrm{C}$ \\
\hline Currents of transmission lines & $\mathrm{C}$ \\
\hline
\end{tabular}

${ }^{1}$ C: Continuous; ${ }^{2}$ D: Discrete; ${ }^{3}$ B: Binary.

- Cost function considering the valve-point effect

In reality, the input flow of vapor in thermal power plants should be adjusted to control the output power. Regulating the flow of vapor is carried out by implementing a set of valves, which augment some ripples into the conventional cost function, Equation (6). In this connection, Figure 4 portrays the aforementioned phenomenon termed as Valve-Point Effect (VPE), which can be modeled as (13).

$$
C_{V P E}(X)=\min \sum_{i=1}^{N_{G}}\left(a_{i} P_{G_{i}}^{2}+b_{i} P_{G_{i}}+c_{i, 1}+\left|d_{i} \sin \left(e_{i}\left(P_{G_{i}}^{\min }-P_{G_{i}}\right)\right)\right|\right)
$$

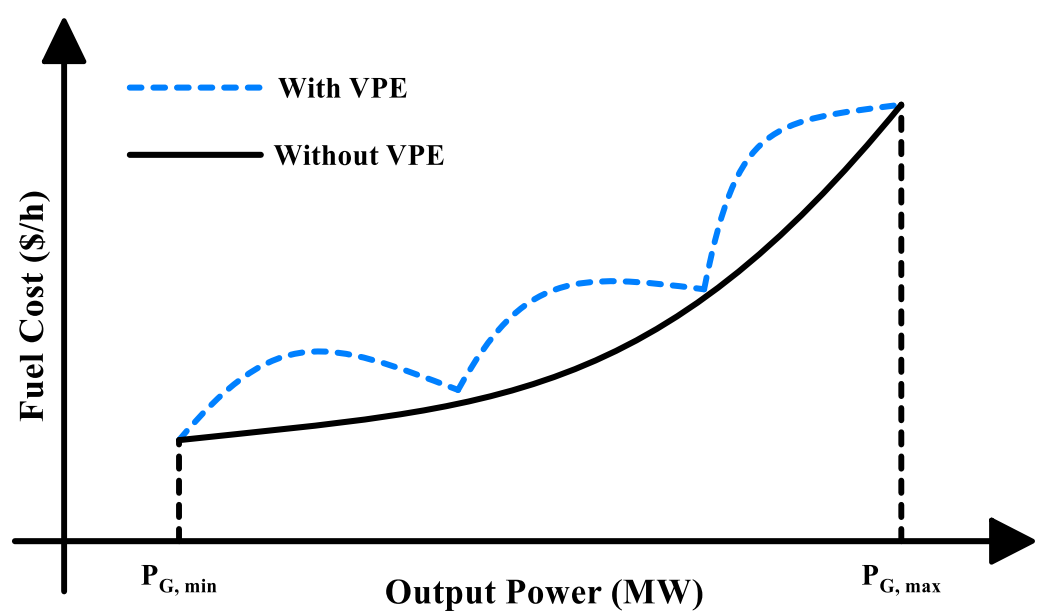

Figure 4. Curves of the cost function with and without the presence of Valve-Point Effect.

- Cost function considering the Multi-Fuel Operation

Generally, power plants consist of various kinds of production units that might utilize different types of fuels such as fossil fuels and natural gas, to name just two. In these conditions, power plants objective function with multiple fuels, namely the Multi-Fuel Operation (MFO), which is a piecewise polynomials function (see Figure 5). The MFO can be mathematically written as (14) and (15) without and with considering VPE, respectively. 


$$
C_{M F O}(X)=\min \left\{\begin{array}{cc}
\text { Fuel Type } 1 ; \sum_{i=1}^{N_{G}}\left(a_{i, 1} P_{G_{i}}^{2}+b_{i, 1} P_{G_{i}}+c_{i, 1}\right) & P_{G_{i}}^{\text {min }} \leq P_{G_{i}} \leq P_{G_{i, 1}} \\
\text { Fuel Type 2; } \sum_{i=1}^{N_{G}}\left(a_{i, 2} P_{G_{i}}^{2}+b_{i, 2} P_{G_{i}}+c_{i, 2}\right) & P_{G_{i, 1}} \leq P_{G_{i}} \leq P_{G_{i, 2}} \\
\vdots & \\
\text { Fuel Type } N_{F} ; \sum_{i=1}^{N_{G}}\left(a_{i, N_{F}} P_{G_{i}}^{2}+b_{i, N_{F}} P_{G_{i}}+c_{i, N_{F}}\right) & P_{G_{i,\left(N_{F}-1\right)}} \leq P_{G_{i}} \leq P_{G_{i}}^{\max }
\end{array}\right.
$$

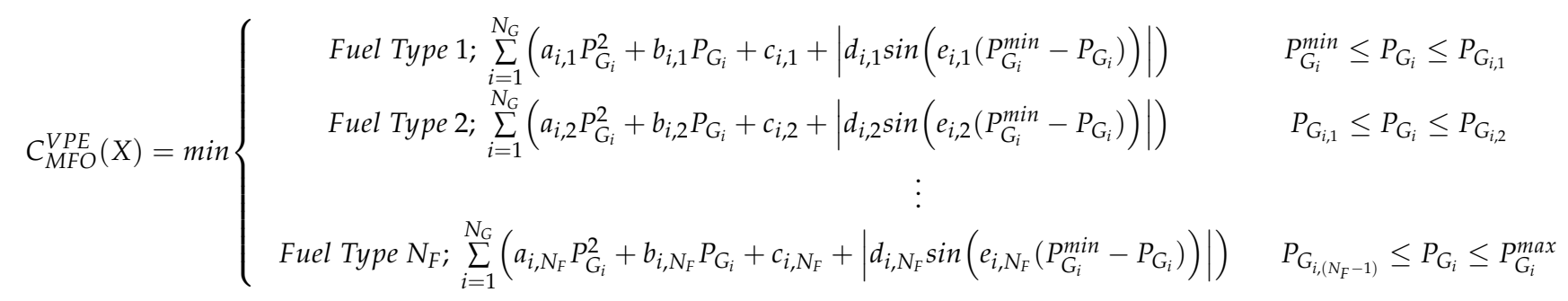

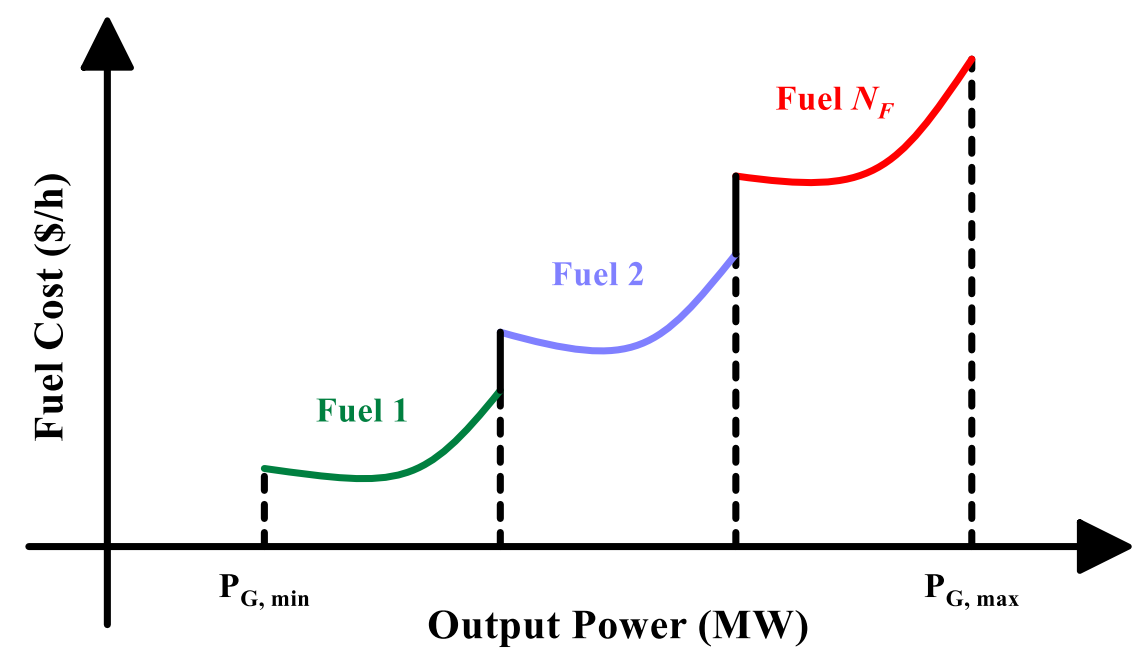

Figure 5. Cost function with multiple fuels.

It is incontrovertibly axiomatic that apart from generating active power in thermal power plants, some contaminants, perilous gases, are emitted into the atmosphere (e.g., $\mathrm{SO}_{x}, \mathrm{NO}_{x}$, and $\mathrm{CO}_{2}$ ). It should be noted that the emission function can be formulated as two different versions, linear and non-linear emission functions, each of which is elaborated hereunder.

- Linear emission function

The linear version for the emission function is written as (16), whose control and state variables are as follows: control variables include the output active power at PV buses apart from the slack one, voltage amplitude at PV buses, settings of transformers' taps, and shunt VAR compensators, while state variables entail the output active power at the slack bus, voltage amplitude at PQ buses, output reactive power of all generators, and transmission line flow.

$$
E_{L}(X)=\min \sum_{i=1}^{N_{G}}\left(\sigma_{i} P_{G_{i}}\right)
$$

- Non-linear emission function. 
The non-linear emission functions can be written as (17) and (18).

$$
\begin{gathered}
E_{N L}(X)=\min \sum_{i=1}^{N_{G}}\left(\gamma_{i} P_{G_{i}}^{2}+\beta_{i} P_{G_{i}}+\alpha_{i}+\xi_{i} e^{\lambda_{i} P_{G_{i}}}\right) \\
E_{N L}(X)=\min \sum_{i=1}^{N_{G}}\left(\gamma_{i} P_{G_{i}}^{2}+\beta_{i} P_{G_{i}}+\alpha_{i}+\xi_{i, 1} e^{\lambda_{i, 1} P_{G_{i}}}+\xi_{i, 2} e^{\lambda_{i, 2} P_{G_{i}}}\right)
\end{gathered}
$$

The point that constraints and limitations oriented to minimizing emission functions can be written as (49) and (50) and also (54)-(61) as equality and inequality constraints and restrictions, respectively.

Recently, power loss has become a pivotal concern in the power industry. The amount of wasted power in the transmission network has been increased by power systems expansion and increasing system demand. Therefore, many researchers have paid a great deal of attention to power transmission loss as an independent objective function in copious studies, which can be formulated as (19).

$$
P_{\text {Loss }}=\min \sum_{l=1}^{N_{L}} G_{l}\left(V_{i}^{2}-V_{j}^{2}-2 V_{i} V_{j} \cos \left(\theta_{i}-\theta_{j}\right)\right)
$$

To minimize the active power losses in the OPF studies, vectors of control and state variables are as follows.

Control variables entail the output active power at PV buses, output reactive power at PV nodes, voltage amplitudes at PV buses, settings of transformer's tap, and shunt VAR compensators.

State variables consist of output active power at the slack bus, voltage magnitude at PQ buses, and transmission line flow.

Analogous to previous objectives, in the realm of solving the OPF problem considering power loss, (19) is minimized subject to (49) and (50) as equality constraints and (54)-(57) as inequality limitations (see Section 2.1.4).

In order to enhance the system security by modifying the voltage profile and also for improving the voltage stability margin, (54)-(57) are considered as equality constraints, and (54)-(60) are considered as inequality constraints in the OPF procedure (see Section 2.1.4).

\section{- Voltage deviation index}

Another function that plays a pivotal role in maintaining the power network's security at an acceptable level is voltage deviation. Electrical equipment is designed to function normally in an optimum operation at their nominal voltage, which is equal to $1.00 \mathrm{p}$.u. Any deviation from this amount can eventuate in decreasing the efficiency, increasing the rate of damage of electronic devices, and severely reducing the life of these implements, to name but a few. Accordingly, voltage deviation is considered to streamline the system's voltage profile by minimizing the sum of voltage deviations at PQ buses from the predefined reference value, which can be expressed as (20).

$$
V D I=\min \sum_{k=1}^{N_{P Q}}\left|V_{k}-V_{k}^{r e f}\right|
$$

In this connection, to handle the OPF problem and minimize the amount of voltage deviation, vectors of control and state variables are elaborated upon as follows: control variables include the output active power of generators and voltage magnitude of buses, while state variables comprise voltage amplitudes and voltage phase angles of PQ buses.

- Voltage stability index

The static voltage stability index is formulated as (21)-(29). It is worth mentioning that (22) is relevant to the Y-bus matrix; (23) indicates network equation according to node admittance matrix; based on (24)-(26), all nodes are classification into two groups, including PV and PQ buses; (27) is voltage in $k^{\text {th }} \mathrm{PQ}$ bus based on superposition theorem; (28) is an important index associated with VSI, in which the static voltage stability margin is obtained using minimal this index; and finally, (29) develops the objective function for enhancing the VSI.

$$
\begin{gathered}
L=\left|1-\sum_{i=1}^{N_{G}} M_{i j} \times \frac{V_{i}}{V_{j}}\right| ; j=N_{G}+1, \ldots, n \\
{[M]=-\left[Y_{L L}\right]^{-1}\left[Y_{L G}\right]}
\end{gathered}
$$




$$
\begin{gathered}
{\left[I_{B}\right]=\left[Y_{B}\right]\left[V_{B}\right]} \\
{\left[\begin{array}{c}
I_{L} \\
I_{G}
\end{array}\right]=\left[\begin{array}{cc}
Y_{L L} & Y_{L G} \\
Y_{G L} & Y_{G G}
\end{array}\right]\left[\begin{array}{c}
V_{L} \\
V_{G}
\end{array}\right]} \\
I_{L}=Y_{L L} \times V_{L}+Y_{L G} \times V_{G} \\
Y_{L L}^{\prime} \times V_{L}+Y_{L G} \times V_{G}=0 \\
V_{L}^{k}=\sum_{i=1}^{N_{G}}\left(\left(Y_{L L}\right)^{-1} Y_{L G}\right)_{k, i} \times V_{G}^{k} \\
L_{j}=\sum_{i=1}^{N_{G}}\left(\left(\hat{Y}_{L L}\right)^{-1} Y_{L G}\right)_{k, i} \\
V S I=\max \left(L_{j}\right)
\end{gathered}
$$

To solve the voltage stability-oriented OPF problem, the vectors of control variables as well as the vector of state variables are introduced as follows: control variables include the output power of generators and voltage amplitudes of buses, while state variables comprise voltage amplitudes and voltage phase angles of the PQ buses.

By extending the OPF problem over multiple time slots, the problem turns into dynamic optimal power flow (DOPF), in which an inter-temporal relation between quantities in different time intervals is taken into consideration, which is introduced in detail in Section 2.1.2.

\subsubsection{Dynamic Optimal Power Flow}

The mathematical model for the DOPF problem, which is a complex optimization problem in short-term planning by deciphering the power generation schedule in $24 \mathrm{~h}$ [13], can be written as (30)-(40). It is necessary to note that the vectors of control and state variables are the same as the OPF problem, which has been extended over multiple time intervals. In this connection, equality and inequality constraints and limitations are as (49)-(61), which are presented in Section 2.1.4.

- Simplified dynamic cost function

The cost function for solving the DOPF problem without practical constraints can be represented as (30).

$$
\begin{gathered}
F(X)=\min \sum_{t=1}^{T} \sum_{i=1}^{N_{G}}\left(a_{i}\left(P_{g i}^{t}\right)^{2}+b_{i}\left(P_{g i}^{t}\right)+c_{i}\right) \\
X=\left[P_{g}, V_{g}, T A P, Q_{c}\right]_{1 \times n} \\
P_{g}=\left[P_{g 1}, P_{g 2}, \ldots, P_{g\left(N_{G}-1\right)}\right]_{1 \times\left(\left(N_{G}-1\right) \times T\right)} \\
P_{g i}=\left[P_{g i}^{1}, P_{g i}^{2}, \ldots, P_{g i}^{t}, \ldots, P_{g i}^{T}\right]_{1 \times T} \\
V_{g}=\left[V_{g 1}, V_{g 2}, \ldots, V_{g N_{G}}\right]_{1 \times\left(N_{G} \times T\right)} \\
V_{g i}=\left[V_{g i}^{1}, V_{g i}^{2}, \ldots, V_{g i}^{t}, \ldots, V_{g i}^{T}\right]_{1 \times T} \\
T A P=\left[T A P_{1}, T A P_{2}, \ldots, T A P_{N_{T}}\right]_{1 \times\left(N_{T} \times T\right)} \\
T A P_{m}=\left[T A P_{m}^{1}, T A P_{m}^{2}, \ldots, T A P_{m}^{t}, \ldots, T A P_{m}^{T}\right]_{1 \times T} \\
Q_{c}=\left[Q_{c 1}, Q_{c 2}, \ldots, Q_{c N_{c}}\right]_{1 \times\left(N_{c} \times T\right)} \\
Q_{c n}=\left[Q_{c n}^{1}, Q_{c n}^{2}, \ldots, Q_{c n}^{t}, \ldots, Q_{c n}^{T}\right]_{1 \times T} \\
n=\left(N_{T}+N_{c}+N_{G}+\left(N_{G}-1\right)\right) \times T
\end{gathered}
$$

- Dynamic cost function with VPE

As pointed out earlier, in practice, thermal power plants are equipped with several valves to regulate the pressure of vapor, which consequently culminate in a tangible loss of power [11]. In addition, this objective function minimizes total operation cost in only one parochial area. Therefore, 
an accurate model in this realm of study should take the VPE into account. In this connection, the cost function is modified to include the aforementioned phenomenon; (41) expresses this function.

$$
f(X)=\min \sum_{t=1}^{T} \sum_{i=1}^{N_{G}}\left(a_{i}\left(P_{g i}^{t}\right)^{2}+b_{i}\left(P_{g i}^{t}\right)+c_{i}+\left|d_{i} \times \sin \left(e_{i} \times\left(P_{g i}^{\min }-\left(P_{g i}^{t}\right)\right)\right)\right|\right)
$$

\subsubsection{Optimal Reactive Power Dispatch}

For many years, in modern-day power systems, this sub-problem of the OPF problem has been solved to obtain a trade-off between security and economic conditions. In addition, the reactive power dispatch problem deals with the allocation of reactive power sources aiming at minimizing the power losses and keeping all of the voltage magnitudes within their acceptable limits while satisfying multiple equality and inequality constraints [14]. It is notable that quite a few objective functions are considered by researchers in this avenue of research (see Figure 3), few of which are introduced in the sequel. In this regard, it is worth stating that these objectives are optimized subject to (49) and (50) and also (55) and (58)-(61) as equality and inequality constraints, respectively (see Section 2.1.4). In addition, the vector of control and state variables are elaborated upon as follows: control variables consist of output active power of generators, tap ratio of transformers, and switched shunt reactive devices, while state variables entail voltage amplitudes at load buses, voltage phase angle at every bus, output reactive power of generators, and line flows.

- $\quad$ VAR cost

A conventional model for the cost of VAR resources, which is a more practical format for VAR cost, can be written as (42), in which $C_{0}$ is fixed cost (USD/h) and $C_{1}\left(Q_{C}\right)$ incremental/variable cost ( $\frac{\$}{\text { MVAR }}$.hour $)$.

$$
C_{V A R}=\min \left\{C_{0}+C_{1}\left(Q_{C}\right)\right\}
$$

- $\quad$ VAR cost and total fuel cost

Minimizing the VAR cost cannot individually satisfy the other aspects, such as minimizing the total fuel cost. As a cogent alternative in this connection, the late function can be added to (42) to cope with the aforementioned issue at the same time, which is written hereunder as a combination of (5) and (42).

$$
C_{V A R}^{\text {Fuel }}=\min \left\{C_{0}+C_{1}\left(Q_{C}\right)+\sum_{i=1}^{N_{G}}\left(a_{i} P_{g i}^{2}+b_{i} P_{g i}+c_{i}\right)\right\}
$$

It should be noted that in order to minimize (43), some extra practical constraints (e.g., VPE and MFO) can be considered as well, which is handled by implementing other versions of the cost function rather than (5), which are introduced in the sequel.

- $\quad$ VAR cost and cost of active power losses

The objective function for solving the ORPD problem by optimizing the VAR cost and also cost of active power losses at the same time can be written as (44).

$$
C_{V A R}^{\text {Loss }}=\min \left\{C_{0}+C_{1}\left(Q_{C}\right)+\sum_{h=0}^{N_{O C}} C_{2}\left(P_{\text {loss }}\right)\right\}
$$

where $C_{2}\left(P_{\text {loss }}\right)$ is the cost of active power losses, $N_{O C}$ is number of operation cases, and $h=0$ is related to base case.

- $\quad$ Active power transmission losses

The active power transmission losses as an objective function in solving the ORPD problem can be mathematically written as (45), which is the same as OPF problems.

$$
\operatorname{Loss}(X)=\min \sum_{l=1}^{N_{L}} G_{l}\left(V_{i}^{2}-V_{j}^{2}-2 V_{i} V_{j} \cos \left(\theta_{i j}\right)\right)
$$

- Voltage deviation

The VDI in the ORPD studies is the same as OPF studies, which is represented here as (46).

$$
V D I=\min \sum_{k=1}^{N_{P Q}}\left(V_{k}^{\max }-V_{k}\right)
$$


- Voltage stability

In the area of solving the ORPD and also reactive power planning (RPP) problems, the stability margin (SM) can be formulated as (47); consequently, the resulting objective function for enhancing the system stability is as (48).

$$
\begin{gathered}
S M=\frac{\sum_{k=1}^{N_{P Q}}\left(S_{k}^{c}-S_{k}^{n}\right)}{S_{k}^{c}} \\
V S I=\min (1-S M)
\end{gathered}
$$

where $S_{k}^{n}$ and $S_{k}^{c}$ are loads (MVA) in $k^{\text {th }} \mathrm{PQ}$ bus at normal and critical states, respectively, which are depicted in Figure 6, points A and B, correspondingly.

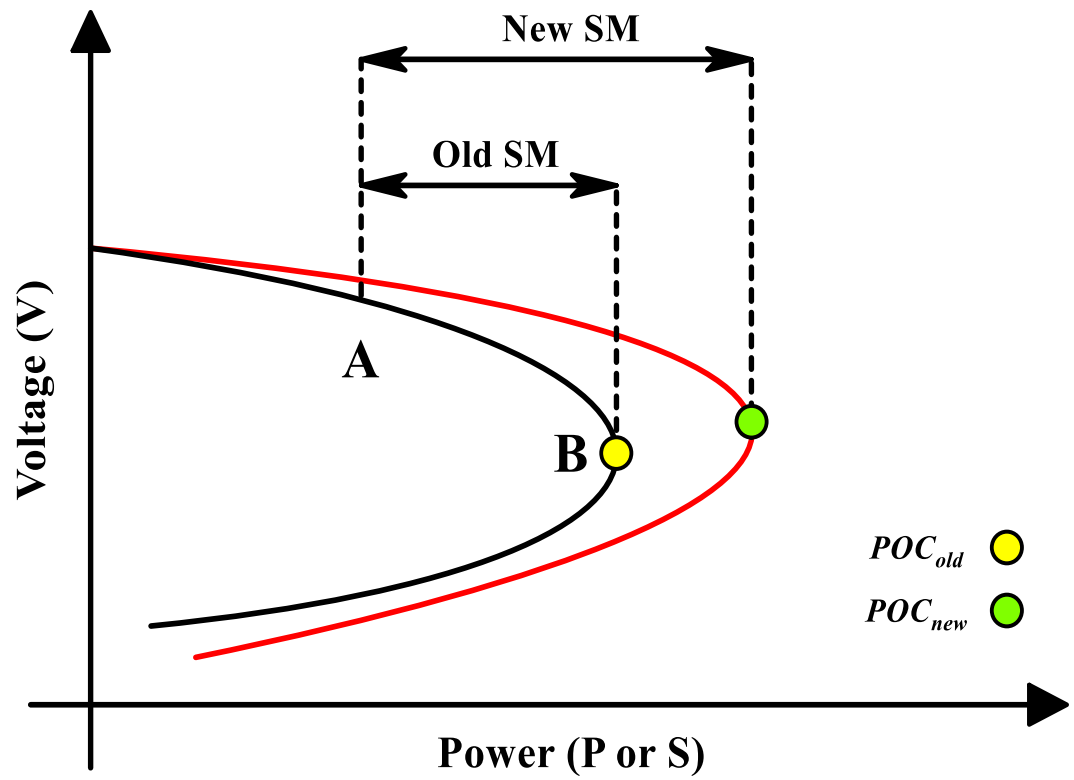

Figure 6. The plot of voltage stability characteristics.

It is necessary to note that Figure 6 is known as the voltage stability curve in which the nose point of the P-V plot is termed as point of collapse (POC). In the POC, the voltage is dropped very fast with an increase in system load. In this regard, and according to Figure 6, the black plot shows the curve of voltage stability in the initial state and before any VAR compensation processes. Further, the red curve illustrates the curve of voltage stability after VAR compensation. In addition, POCs are portrayed for both operations, before and after VAR compensations, by $P O C_{\text {old }}$ and $P O C_{\text {new }}$, respectively.

\subsubsection{Constraints and Limitations}

This section introduces a set of constraints and restrictions to be considered for solving each of the presented problems (e.g., OPF, DOPF, and ORPD), which are approximately the same with the exception that for solving OPF and ORPD, the time interval $t$ must not be considered in the constraints, which are elaborated upon in the following sub-sections.

- $\quad$ Equality constraints

Equality constraints reflect the physics of the power grid, which can be expressed as (49)-(53).

$$
\begin{gathered}
P_{i}^{t}=P_{g i}^{t}-P_{d i}^{t}=\sum_{j=1}^{N_{B}} V_{i}^{t} V_{j}^{t}\left(G_{i j} \cos \left(\theta_{i j}^{t}\right)+B_{i j} \sin \left(\theta_{i j}^{t}\right)\right) \\
Q_{i}^{t}=Q_{g i}^{t}-Q_{d i}^{t}=\sum_{j=1}^{N_{B}} V_{i}^{t} V_{j}^{t}\left(G_{i j} \sin \left(\theta_{i j}^{t}\right)-B_{i j} \cos \left(\theta_{i j}^{t}\right)\right) \\
i, j=1,2, \ldots, N_{B} \\
t=1,2, \ldots, T \\
\theta_{i j}^{t}=\theta_{i}^{t}-\theta_{j}^{t}
\end{gathered}
$$


- Inequality constraints

Inequality constraints represent technical obligations dealt with power systems and equipment, which can be expressed as (54)-(61).

$$
\begin{gathered}
P_{g i}^{\text {min }} \leq P_{g i}^{t} \leq P_{g i}^{\text {max }} ; i=1,2, \ldots, N_{G} \\
Q_{g i}^{\text {min }} \leq Q_{g i}^{t} \leq Q_{g i}^{\text {max }} ; i=1,2, \ldots, N_{G} \\
\quad\left|P_{i j}^{t}\right| \leq P_{i j}^{\max } \\
V_{i}^{\text {min }} \leq V_{i}^{t} \leq V_{i}^{\text {max }} ; i=1,2, \ldots, N_{G} \\
V_{k}^{\text {min }} \leq V_{k}^{t} \leq V_{k}^{\text {max }} ; k=1,2, \ldots, N_{P Q} \\
V_{b}^{\text {min }} \leq V_{b}^{t} \leq V_{b}^{\text {max }} ; b=1,2, \ldots, N_{B} \\
Q_{c n}^{\text {min }} \leq Q_{c n}^{t} \leq Q_{c n}^{\text {max }} ; n=1,2, \ldots, N_{c} \\
T A P_{m}^{\text {min }} \leq T A P_{m}^{t} \leq T A P_{m}^{\text {max }} ; m=1,2, \ldots, N_{T}
\end{gathered}
$$

\section{- $\quad$ Prohibited operating zones}

Power plants might not be capable of functioning in specific operating regions, which are termed as prohibited operating zones (POZs). This aforementioned phenomenon is due to metal fatigue in turbine blades and/or progressive manner of vibrations in the shaft bearing at certain operating regions. Typically, the input-output curve of the output power of generators with POZ is not absolutely continuous, which is illustrated in Figure 7.

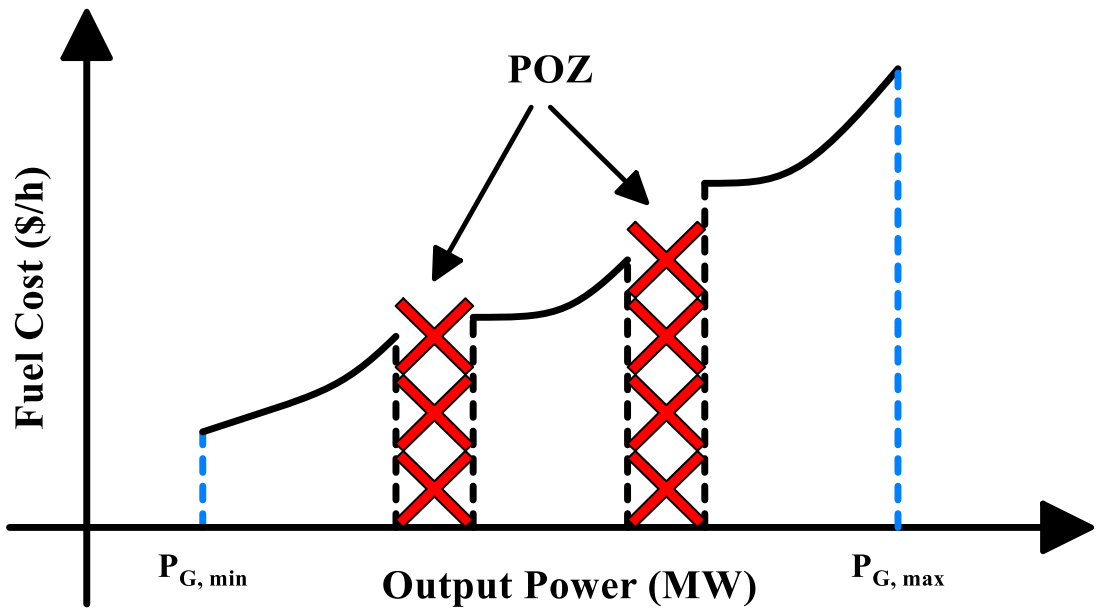

Figure 7. Cost function with disjoint operating zones.

According to Figure 7, the POZs can be mathematically written as (62).

$$
\begin{gathered}
P_{G_{i, t}} \in\left\{\begin{array}{c}
P_{G_{i}}^{\min } \leq P_{G_{i, t}} \leq P_{G_{i, 1}}^{L} \\
P_{G_{i,(z-1)}}^{U} \leq P_{G_{i, t}} \leq P_{G_{i, z}}^{L} \\
P_{G_{i, z}}^{U} \leq P_{G_{i, t}} \leq P_{G_{i}}^{\max }
\end{array}\right. \\
i=1,2, \ldots, N_{G} \\
z=2,3, \ldots, Z
\end{gathered}
$$

- Generator ramp-rate

Inasmuch as the output power of practical generators cannot be regulated instantaneously, the functioning range of all in-service units must be circumscribed by their Ramp-Rate (RR) limits. As a result, the output power of generators in consecutive time slots depends on one another, which can be written as follows.

$$
\begin{aligned}
& P_{g i}^{t}-P_{g i}^{(t-1)} \leq U R_{i} \\
& P_{g i}^{(t-1)}-P_{g i}^{t} \leq D R_{i}
\end{aligned}
$$


In this regard, modified production boundaries considering RR are provided in (67).

$$
\max \left(P_{g i}^{\min }, U R_{i}-P_{g i}^{t}\right) \leq P_{g i}^{t} \leq \min \left(P_{g i}^{\max }, P_{g i}^{(t-1)}-D R_{i}\right)
$$

\subsection{Multi-Objective Approach}

The environmental issues due to the US environmental protection agency and also restructuring process in the power industry have resulted in reconfiguring the operation policy of power networks, which must consider different objectives at the same time. To cope with the aforementioned issue, the under-studied problems should be scrutinized as Multi-Objective Optimization Problems (MOOPs), which cover different dimensions and recognize an acceptable trade-off among them $[15,16]$. In this connection, for getting closer to the real circumstances of power grids, all reviewed objective functions in this paper, which are presented in detail in Section 2.1, can be optimized simultaneously as two-, three-, and four-dimensional problems (refer to Section 4 for more information). Notwithstanding, different objective functions may not be in accord with one another and even may be conflicting with each other. Generally speaking, two common tactics are utilized to solve the MOOPs with conflicting objective functions, which are as follows.

- Weighted sum or penalty factor technique;

- $\quad$ Pareto optimal technique.

\section{Heuristic Algorithms Applied to the OPF and ORPD Problems}

This section tries to introduce and concisely review all of the available evolutionary algorithms that have been employed by researchers to solve one of the problems of the OPF family. A detailed investigation has been accomplished accordingly, which is elaborated upon in Section 4. It should be noted that all of the reviewed algorithms start their iterative procedures by randomly generating a matrix of the initial population, each member of which indicates a valid solution for the OPF problem and has a dimension based on the number of decision variables of the problems. To have a better perspective, interested readers are directed to [10] for an illustrative example about the relationship between decision variables of a typical optimization problem and the population size. After generating the initial population associated with a typical optimization algorithm (e.g., PSO, HBMO, SFLA, etc.), each member of the population (e.g., bird in PSO, bee in HBMO, frog in SFLA, etc.) moves toward the optimal solution in the search space until the acceptable convergence occurs of one of the stopping criteria reaches. The evolution process is accomplished by applying a set of laws/equations/inequalities, which are different from algorithm to algorithm based on their qualitative differences. For example, in PSO algorithm, each member (e.g., bird) of the initial population tries to update its position and velocity toward the optimal solution in the search space; hence, two equations related to position and velocity are assigned to each member to model the behavior of birds in finding the destination [1]. However, in SFLA, first the initial population is divided into several subsets called memeplexes, which is also considered as an independent culture of members (i.e., frogs) performing a local search for food. Then, the information about the location of food is exchanged between different memeplexes through the shuffling process. Therefore, in SFLA, only one equation (i.e., the position of frogs) is assigned to each member [10]. To obtain a better perspective, Figure 8 demonstrates a generic flowchart of a heuristic/metaheuristic algorithm to the OPF problem.

\section{- $\quad$ Genetic Algorithm}

One of the most well-known and efficient optimization algorithms employed to solve a variety of optimization problems is the Genetic Algorithm (GA). The GA strives to find an optimal solution to any OPF and ORPD problems using methods that imitate the natural evolution existing in the real world, such as inheritance, mutation, selection, and crossover.

Efficient Parallel GA (EPGA) combined with practical rules was successfully implemented in [17] to improve the quality of optimal solutions of the OPF problem with non-smooth cost function under different loading circumstances. Enhanced GA (EGA) was proposed in [18] to solve the OPF, taking into account the concept of incremental power flow model, which is based on sensitivities, leading to a noticeable reduction in the CPU time. An EGA-oriented computation algorithm for handling the Multi-Objective OPF (MOOPF) problem has been proposed in [19], where a new Decoupled Quadratic Load Flow (DQLF) was combined with EGA to find better optimal solutions. The proposed multi-objective method managed to determine diverse Pareto Optimal Front (POF) for the OPF problem in a reasonable CPU time. An Improved GA (IGA) was proposed in [20] for 
solving the OPF problem under both normal and contingent operation periods. The proposed IGA, with the dynamical hierarchy of the coding system, was capable of coding a copious number of control variables in real power networks within a reasonable length string. A comparative study of MOOPF problem based on Particle Swarm Optimization (PSO), Evolutionary Programming (EP), and GA was developed in [21], where different objective functions (e.g., cost, active power loss, voltage stability index, and emission) were considered. Moreover, a new approach combining NewtonRaphson and Fast-Decouple was employed to decrease the execution time of the calculations. A voltage stability constrained optimal power flow model using Non-Dominated Sorting GA-II (NSGAII) was introduced in [22], where different effective genetic operations, crossover, and mutation operators are employed. An OPF for large-scale power systems with shunt Flexible Alternating Current Transmission Systems (FACTS) devices using EPGA was presented in [23]. Since the main counterpoints of GAs are the high execution time and deteriorating the quality of the solution with practical large-scale OPF problems, the aforementioned study proposed an EPGA in which the length of the original chromosome was reduced successively according to the decomposition level and adjusted with the topology of the new partition. Furthermore, GA has been employed to solve the FACTS allocation using the OPF problem. Allocation of Thyristor Controlled Phase Shifting Transformer (TCPST) was carried out using the GA algorithm and OPF equations [24]. In a similar study, a GA was applied to allocate capacitors and Voltage Regulators (VRs) at distribution networks using the OPF problem [25]. The specification for the kind of capacitor bank, which can be fixed or automatic, and the reactive power (KVAR), as well as the allocation of the VRs were handled by GA such that the OPF was responsible for the solution of the power balance equations and tap adjustments of the VRs. An ORPD in the AC-DC power system, including High Voltage Direct Current (HVDC), was investigated in detail in [26]. It was shown that the GA converges reliably and rapidly to the reasonable optimal solutions in comparison with other methods.

- Particle Swarm Optimization

Inspired by the social behavior of animals (e.g., fish schooling or bird flocking), Particle Swarm Optimization (PSO) as a swarm intelligence-based optimization algorithm was introduced by Eberhart and Kennedy in 1995 [27]. Over the years, PSO has been applied to solve both discrete and continuous optimization problems since its presentation.

A PSO algorithm was employed to solve the OPF problem in [28], where the global and local exploration capability of the PSO algorithm was used to attempt in recognizing the most cogent and optimal setting of the control variables. PSO with an Aging Leader and Challengers (ALC-PSO) was implemented in [29] to find a high-quality solution to the OPF problem in a system equipped with FACTS components. A fuzzy evolutionary and swarm optimization was presented in [30] to handle OPF problems. This approach employed an integration of the Fuzzy Interface System (FIS) along with GA and PSO algorithms for the optimal setting of the problem's control variables. The performance of a novel configuration of a Fuzzy Adaptive Heterogeneous ComprehensiveLearning PSO (FAHCLPSO) to solve the ORPD problem was studied in detail in [31], considering different objective functions, including active power transmission losses and total voltage deviations of the system. In [32], a PSO was implemented as a potent tool for loss reduction study. The proposed approach in [32] comprises a developed version of the traditional OPF problem with loss minimization objective function aiming at improving the voltage stability index. A fuzzy-based hybrid PSO approach for solving the OPF problem with uncertainties was introduced in [33], where the forecast load demand and also wind speed errors have been taken into account so as to handle the OPF problem more accurately. In [34], an Evolutionary PSO (EPSO) approach was proposed to handle the OPF problem considering up- and down-spinning reserves and the operational constraints of the generation unit. The effects of wind generation on power system operation and planning were investigated, as well. An Evolving Ant Direction PSO (EADPSO) Algorithm for solving the OPF problem with non-smooth and non-convex generator cost coefficients was presented in [35], where an ant colony search approach was used to find a suitable velocity updating operator for PSO. In order to update the velocities for PSO, five velocity updating operators were implemented, as well. 


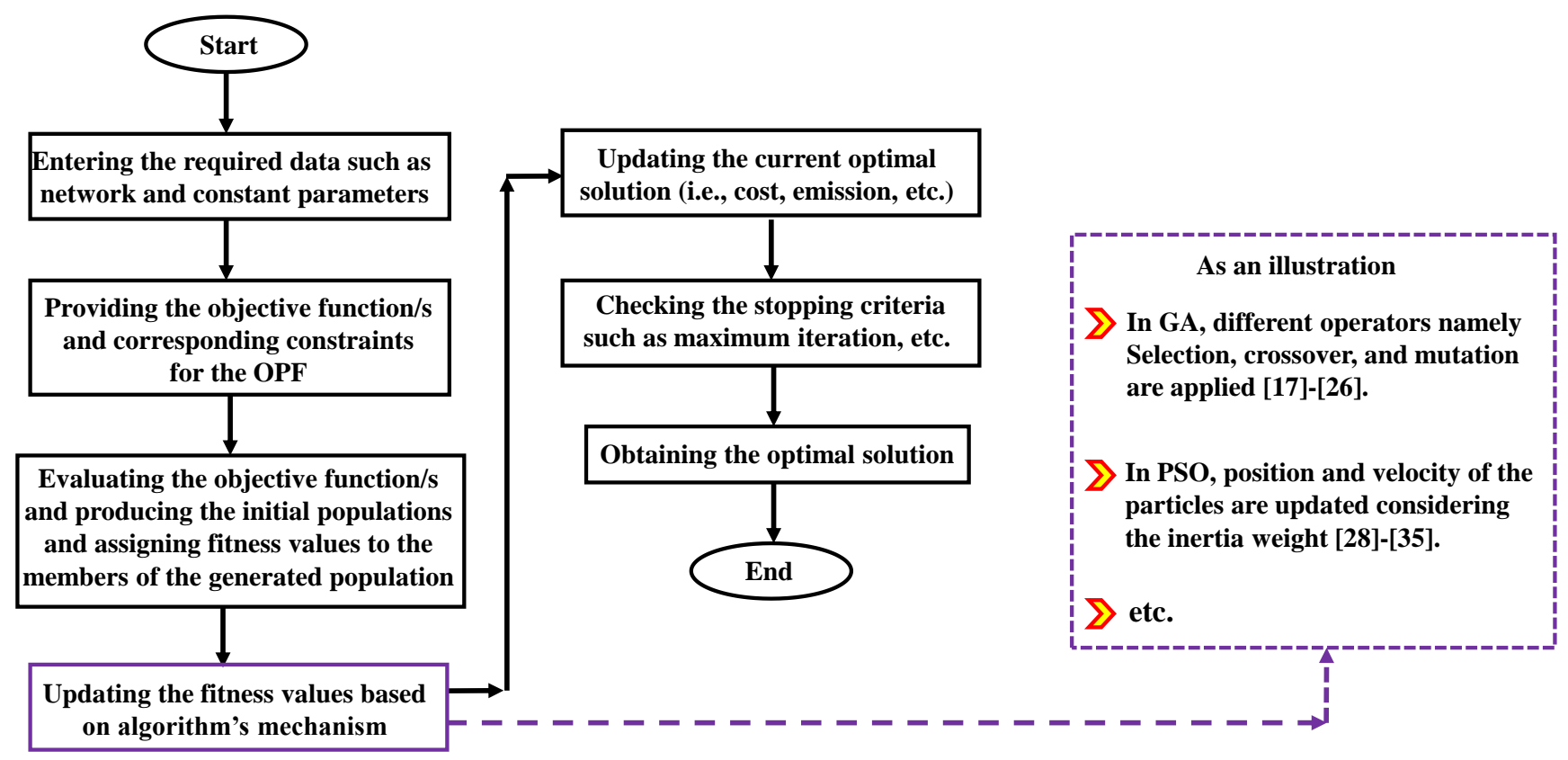

Figure 8. Flowchart associated with all of the reviewed optimization algorithms.

\section{- Honey Bee Mating Optimization}

Inspired by the foraging behavior of bees, bee algorithms are a class of meta-heuristic optimization algorithms. A few variants exist in the literature, including Honey Bee Algorithm, Artificial Bee Colony, Bee Algorithm, Virtual Bee Algorithm, and Honeybee Mating Algorithms. In this section, various bee algorithms are reviewed.

Modified Honey Bee Mating Optimization (MHBMO) was presented in [36] to solve the Dynamic OPF (DOPF) problem in power systems. Non-linear characteristics of the generators and also practical restrictions, such as ramp rate constraints, transmission constraints, valve point effect, and non-linear cost functions, were considered for simulating realistic conditions. A fuzzy-based Modified Artificial Bee Colony (MABC) Algorithm was proposed in [37] to deal with the OPF problems, including both discrete and continuous variables. The OPF problem was formulated as a MultiObjective Optimization Problem (MOOP), where four objective functions such as fuel cost, emission, power losses, and voltage deviation were considered individually and simultaneously. An Artificial Bee Colony (ABC) Algorithm was implemented in [38] as the main basic optimizer to solve the OPF problem, where the control variables consist of both continuous and discrete variables. In addition, different objective functions such as convex and non-convex fuel costs, active power loss, voltage profile improvement, voltage stability, and emission were scrutinized in this study. An efficient OPF model including an offshore wind farm and Plug-in Electric Vehicles (PEVs) connected to the grid was solved by Gbest guided ABC (GABC) Algorithm in [39]. The stochastic nature of wind power and uncertainties in the EV owner's behavior were suitably modeled by statistical models available in the literature. Moreover, the same approach was used in [40] to solve the OPF problem incorporating stochastic wind power. In [41], a MOOPF problem was solved by two popular meta-heuristics algorithms, namely, the ABC and the Teaching-Learning-Based Optimization (TLBO) Algorithm. The proposed multi-objective method was based on a decomposition approach, where the MOOP was decomposed into a number of scalar optimization sub-problems that were simultaneously optimized. An enhanced version of the ABC (EABC) Algorithm was implemented in [42] to solve a Dynamic Security Constrained OPF (DSCOPF) problem as an extension of the SCOPF problem over multiple time intervals. The proposed EABC has high exploration capability and could discover different areas of the search space. The authors in [43] proposed a Multi-Hive Multi-Objective Bee Algorithm ( $\left.\mathrm{M}^{2} \mathrm{OBA}\right)$ to solve OPF problems. This algorithm extended the original ABC algorithm to handle the multi-objective and cooperative mode by combining external archive, comprehensive-learning, greedy selection, crowding distance, and cooperative search strategy. The proposed algorithm used the concept of Pareto dominance and comprehensive learning mechanisms to determine the flight direction of a bee and maintain non-dominated solution vectors in an external archive based on greedy selection and crowing distance strategies. An improved Hybrid Multi-Objective ABC algo- 
rithm (HMOABC) was presented in [44] for solving the multi-objective OPF problem. The main idea of HMOABC was to extend the original $A B C$ algorithm to the multi-objective and cooperative mode by combining the Pareto dominance and also the divide-and-conquer approach. A Security and Transient Stability Constrained OPF (STSCOPF) problem was solved by a Chaotic ABC (CABC) Algorithm in [45]. The STSCOPF can be illustrated as an extended OPF problem with additional line loading and rotor angle inequality constraints. An ABC algorithm with Quantum Theory and the Chaotic Local Search Strategy (QCABC) was proposed in [46] to solve the OPF problem. A new multi-objective Chaotic Parallel Vector Evaluated Interactive Honey Bee Mating Optimization (CPVEIHBMO) was proposed in [47] to find the feasible optimal solution for the multi-objective ORPD problem considering operational constraints of the generators. The proposed algorithm was applied to find the settings of continuous and discrete control variables such as tap positions in the transformers, generator voltages, and the size of reactive compensation components to optimize three different objective functions with qualitative differences including voltage deviation, the total voltage stability, and active power loss. ABC algorithm was implemented in [48] to solve the ORPD problem aiming at minimizing the active power loss in power systems. The authors stated that the advantage of the $\mathrm{ABC}$ algorithm is that it does not require parameter tuning because it is challenging to determine external parameters such as cross-over rate and mutation rate, as in the case of GA and Differential Evolution (DE) method. The other advantage is that the global search ability of the algorithm is implemented by introducing a neighborhood source production mechanism that is similar to the mutation process.

- $\quad$ Shuffled Frog Leaping Algorithm

Shuffle Frog Leaping Algorithm (SFLA) mimics the metaphor of natural biological evolution that is based on the population of frogs in nature searching for food. This algorithm was introduced by Eusuff, Lansey, and Pasha in 2006 for the first time [49]. The SFLA is a stochastic search algorithm in which the iterative process begins with an initial population of frogs, whose characteristics represent the decision variables of the optimization problem.

A Modified SFLA (MSFLA) was proposed in [50] for solving the MOOPF problem. This study targeted economic and emission issues that privileged a mutation operator in order to reduce the processing time and improve the quality of the solutions. An MSFLA for MOOPF problem with FACTS devices was studied in [51]. The algorithm employed a new mutation strategy to increase the efficiency of the original SFLA. The SFLA was also applied to solve the OPF in an AC-DC power system, including both High Voltage Alternating Current (HVAC) and HVDC transmission lines [52]. A hybrid SFLA has been implemented to solve the ORPD problem in [53]. In order to fully exploit the promising solution region, a local search algorithm termed as Nelder-Mead (NM) Algorithm was integrated with SFLA. The most important benefit of the proposed method, based on the authors' claim, was with higher speed of convergence to a better solution.

\section{- $\quad$ BAT Algorithm}

The bat-inspired algorithm is a meta-heuristic optimization algorithm developed by Xin-She Yang [54]. It is according to the echolocation behavior of micro bats with varying pulse rates of emission and loudness.

A BAT algorithm was presented in [55] to solve the OPF problem for generation reallocation with Unified Power Flow Controller (UPFC). The objective of this study was to minimize active power losses in a power system with/without considering the UPFC devise. Further, minimization of active power losses was realized by considering the generator output, voltage magnitude at generator buses, and reactive power injection of compensators. A security-constrained economic load dispatch at the presence of an Interline Power Flow Controller (IPFC) was investigated by a modified BAT algorithm in [56]. In this approach, detailed mathematical modeling of IPFC and the impact of its optimal location were studied.

- $\quad$ Firefly Algorithm

Firefly Algorithm (FA) is a nature-inspired optimization algorithm that functions according to the flashing behavior of fireflies. This algorithm was proposed by Yang for the first time [56].

An Enhanced FA (EFA) was proposed in [57] to solve a multi-objective optimal active/reactive power dispatch with considering uncertainties, where active and reactive power dispatches were considered at the same time. Load tap changer positions of transformers, reactive power injection of capacitor banks, and voltage amplitudes of slack bus and also all of the PV buses were controlled to reduce the transmission line losses and voltage deviations of load buses in reactive power dispatch. A mutation strategy and a local random search were implemented to improve the algorithm's 
searching performance. The application of FA on the OPF problem in the presence of a simplified impedance UPFC model was presented in [58]. In [59], authors utilized an FA so as to overcome the nonlinearity of the OPF problem integrated with static VAR compensator. Different objective functions, including total fuel cost, total power losses, total voltage deviation, and loading margin stability, were considered in the mentioned study. A hybrid FA and NM simplex algorithm was presented in [60] to solve the ORPD problem in the power system. NM simplex is an efficient local search method that was implemented in this study in order to overcome the counterpoints of FA, such as trapping in local optima, for instance. The proposed search algorithm was scrutinized in detail to find optimal settings of generator voltages, tap positions of tap changing transformers, and VAR output of shunt capacitors so as to minimize two different and conflicting objective functions.

- Gravitational Search Algorithm

Gravitational Search Algorithm (GSA) is a heuristic optimization method oriented toward the law of gravity and mass interactions [61]. According to Newtonian gravity, "Every particle in the universe attracts every other particle with a force that is directly proportional to the product of their masses and inversely proportional to the square of the distance between them".

A Non-Dominated Sorting Multi-Objective Opposition-Based GSA (NSMOOGSA) was presented in [61] to handle different versions of the OPF problem, single- and multi-objective optimal OPF. The oppositional learning concept was utilized to enhance the convergence acceleration process, while the non-dominated sorting with crowding distance algorithm was employed to manage the obtained Pareto optimal fronts. In [62,63], the GSA was implemented to recognize the optimal solution to the OPF problem, where different objective functions such as fuel cost, loss, and voltage deviation were considered in these approaches. A GSA-based approach was proposed in [64] to solve the OPF problem in a distribution network with several Distributed Generation (DG) sources. Further, the OPF problem was formulated as a non-linear optimization problem to minimize different objective functions, including the fuel cost of DG sources and power loss in the network. An Opposition-Based GSA (OGSA) was introduced in [65] to solve the OPF problem integrated with FACTS devices (i.e., Thyristor Controlled Series Capacitor (TCSC) and Thyristor Controlled Phase Shifter (TCPS)) in order to optimize three objective functions (i.e., cost of generation, emission, and active power transmission loss).

- Imperialistic Competition Algorithm

Imperialist Competitive Algorithm (ICA) is a computational method for solving different types of optimization problems. From a specific point of view, ICA can be thought of as the social counterpart of GAs. This algorithm has two main operators, including Assimilation and Revolution, both of which were elaborated upon in [66].

The ICA and Artificial Neural Network (ANN) were employed in [67] to develop a robust and efficient two stage-scheme for solving the Transient Stability Constrained OPF (TSC-OPF) problem. ANN was utilized to predict the rotor-angle transient stability margin, while ICA was used as the main optimizer. A novel hybrid ICA and TLBO method was presented in [68] to solve the OPF problem with non-smooth cost functions. A series of modifications were applied to the assimilation policy rule of ICA to increase the solution quality. A new Multi-Objective Modified ICA (MOMICA) was presented in [69] to solve the MOOPF problem, where different objective functions, including generation cost, emission, and voltage deviation, were studied in detail. The Modified ICA (MICA) was applied to the MOOPF problem in [70]. The interaction effects of colonies on each other were modeled to enhance the local search near the global optima. Furthermore, a series of modifications were implemented to the assimilation policy rule of the ICA method in order to increase the efficiency of ICA.

- Cuckoo Search Algorithm

Cuckoo Search (CS) Algorithm is an optimization method developed by Xin-she Yang and Suash-Deb in 2009 [71]. This algorithm is based on the obligate brood parasitic behavior of some cuckoo species in combination with the levy flight behavior of some birds.

A Cuckoo Optimization Algorithm (COA) was utilized in [72] for solving the OPF problem considering three types of fuel cost such as quadratic cost function, cost function with valve point effect, and multiple fuels. A hybrid CS algorithm was implemented to solve the OPF problem in [73] to find the steady-state operating point, which minimizes the fuel cost. A cross-over method was used to increase the efficiency of the CS algorithm. Improved CS (ICS) Algorithm was presented in [74] for solving the Multi-Objective ORPD (MOORPD) problem. Loss minimization and maximization 
of voltage stability margin were taken as objective functions. A proper strategy for tuning the CS parameters was proposed to increase the efficiency of the algorithm.

- Bacterial Foraging Algorithm

Bacteria Foraging Algorithm (BFA) is a class of biologically stochastic global heuristic search technique, which is oriented toward mimicking the foraging behavior of E. coli bacteria [75].

A Dynamic BFA (DBFA) was introduced in [76] to solve OPF in a dynamic environment. The importance of dynamic optimization comes under the spotlight due to the fact that the demand of the system, the capacity of the generation side, and transmission networks in a power network are almost always in a variable status. Consequently, the static-based methods show deficiency for dynamic environments. A modified BFA was proposed in [77] to solve the OPF problem integrated with wind power penetration. Owing to the intermittent nature of wind flow, solving the OPF problem in a power system with a significant amount of wind power penetration can be challenging, which was thoroughly studied in this study. An Enhanced BFA (EBFA) approach was developed in [78] to handle the OPF problem for power systems equipped with FACTS devices. The BFA was streamlined by including the Nelder-Mead (NM) simplex algorithm for having a better performance. In another study [79], the BFA was applied for solving the multi-objective multivariable OPF problem in the presence of UPFC. The UPFC location, its series injected voltage, and the transformer tap positions were considered as the control variables. Moreover, working out the solution of the SecurityConstrained OPF (SCOPF) problem with wind-thermal generation system was studied by Modified BFA (MBFA) in [80] wherein the main objective was operating the wind-thermal generation system in a cost-effective manner.

- Harmony Search Algorithm

The concept of Harmony Search Algorithm (HSA) has been introduced from the musical process of searching for a "perfect state" of harmony; as an illustration, jazz improvisation searches the best state determined by aesthetic estimation, just as the optimization algorithm explores the search space for finding the best state determined by computing the fitness functions [81].

An Improved Harmony Search (IHS) Algorithm was proposed in [82] for solving different models of the OPF problem, considering both smooth and non-smooth fuel-cost functions. Moreover, the proposed methodology was successfully tested on five different scale test systems. A MultiObjective HS (MOHS) Algorithm was presented in [83] for solving a nonlinear constrained multiobjective OPF problem, where a fast elitist non-dominated sorting was implemented to recognize and manage the Pareto optimal fronts. A mixed-integer nonlinear ORPD problem has been solved by HSA in [84]. The continuous and discrete control variables in this paper include the voltage amplitude of generators (PV buses), tap positions of transformers, and the number of reactive compensation components. Different fitness functions, power transmission loss, voltage stability, and voltage profile were optimized individually in this study.

- Ant Colony Optimization

Ant Colony Optimization (ACO) Algorithm, which was proposed by Dorigo for the first time, is capable of handling various combinatorial complex problems [85]. The ACO algorithms were developed according to the surveillance of real ants, especially their foraging behavior. It is notable that they are almost always blind animals with circumscribed capacities; nevertheless, they are able to find the close proximity between their nest(s) and a source of food without visual clues.

In this connection, the ACO algorithm was implemented in [86] to solve the OPF in an economic dispatch setting. The proposed methodology was introduced to be utilized for maintenance planning with 48 to $24 \mathrm{~h}$ of anticipation. The main strong point of this approach was its low CPU time to handle the OPF problem that allows the use of OPF while a large set of scenarios have to be scrutinized.

- $\quad$ Simulated Annealing

The Simulated Annealing (SA) Approach was first presented by Metropolis [87], which is a randomized gradient descent algorithm that permits probable ascending; therefore, it is able to get rid of undesirable local minima. In addition, this optimization algorithm functions on the basis of the behavior of condensed material at low temperatures, which in reality intimidates the annealing process in both natural freezing/crystallizing liquid or cooling/annealing metal.

The SA algorithms were applied to solve the OPF problem in [88] where the main objective of this study was to confirm the ability to use SA in solving the OPF problem. It should be noted that usually the SA, which has strong local search ability, is hybridized by other optimization algorithms to increase their searching abilities. In this connection, a hybrid PSO-SA algorithm was proposed 
in [11] to solve the OPF problem considering practical restrictions. Interested readers are directed to "Hybrid Algorithms" at the end of this section for extra information regarding different combinations of optimization algorithms to solve the OPF problem.

\section{- $\quad$ Black Hole Algorithm}

The Black Hole Algorithm (BHA) is a new population-based optimization algorithm that was first developed as a new mechanism for increasing the efficiency of the PSO algorithm [89]. Afterward, Hatamlou introduced a novel version of the BHA in [90]. The main idea behind the BHA is the Black Hole $(\mathrm{BH})$ phenomenon, which is an area in the space with a high gravitational force that absorbs any substances (i.e., stars) and even light while they get close to it. In the BHA, stars are considered as a population, the best of which are chosen as the $\mathrm{BH}$ absorbing populations in its vicinity.

An Enhanced Binary BHA (EBBHA) was introduced to solve the probabilistic OPF problem. The $(2 m+1)$ point estimated method was utilized for handling power system uncertainties in the problem. In this connection, the correlation between input and random variables was considered by a correlation matrix. The correlation and fluctuation of system load, photovoltaic (PV), and wind power plants (WPPs), which have a significant impact on transmission lines and voltage amplitude of buses, were taken into account. Moreover, the effect of combined heat and power (CHP) units, VPEs, multiple fuels, and POZs of thermal units were also considered [91]. The BH-Based Optimization (BHBO) approach was implemented to solve the different models of the OPF problem considering various kinds of constraints and limitations [92]. The BHBO is a simple algorithm since it involves only two mathematical equations, which are elaborated upon as follows. The first one indicates how to update the position of stars, and the second one is oriented to how to check whether a star has crossed the event horizon of the $\mathrm{BH}$ or not. Further, the $\mathrm{BHBO}$ algorithm has no inherent parameter to be tuned.

\section{- Teaching-Learning-Based Optimization Algorithm}

The Teaching-Learning-Based Optimization (TLBO) algorithm was developed by Rao [93]. It is designed on the basis of the communication behavior of students in a classroom. In this regard, the teacher plays the role of the best solution obtained so far. Generally, the algorithm is split into two basic operations that are teacher phase and the learner phase, such that the output solutions of the teacher phase are used as the input for the other phase, the learner phase [94].

An improved version of the TLBO algorithm was utilized for solving the non-smooth OPF problem in [95], in which the Levy mutation strategy was implemented to enhance the efficiency of the original algorithm. Another similar study has been conducted for solving the OPF problem by a Modified TLBO (MTLBO) Algorithm, which profits from a self-adapting wavelet mutation strategy that makes the proposed algorithm seek a larger area in the search space for finding the optimal solutions [96]. Moreover, the TLBO algorithm was applied to solve the MOOPF problem in [97]. In order to enhance the convergence speed and also the quality of optimal obtained solution, Quasi-Oppositional-Based Learning (QOBL) was incorporated into the original TLBO algorithm in the aforementioned study where different fitness functions, fuel cost, power loss, voltage stability index, and emission of pollutions were considered. In addition, in another study, the TLBO was proposed to solve the OPF problem with different objective functions such as generation fuel cost, voltage profile enhancement, as well as voltage stability improvement [98]. A Multi-Objective TLBO (MOTLBO) Algorithm based on the non-dominated sorting principle was proposed in [99] for solving the OPF problem where different objective functions, including fuel cost, transmission losses, and voltage stability index, were taken into account. A new TLBO algorithm was proposed in [100] to solve the MOORPD problem for optimizing three different and confliction objective functions such as active power loss, voltage deviation, and voltage stability index. In this work, the QOBL concept was incorporated in the basic TLBO algorithm to accelerate the convergence speed and improve the quality of the optimal solutions.

- $\quad$ Artificial Immune System

Inspired by the fundamentals and processes of the vertebrate immune system, Artificial Immune Systems (AIS) represent a class of computationally intelligent algorithms, which typically exploit the immune system's characteristics of both learning and memory to solve an optimization problem.

Accordingly, probabilistic constrained AIS was proposed in [101] to solve the OPF problem in which an improved evolutionary algorithm based on Cluster-and-Gradient-based AIS (CGbAIS) was implemented to reduce the execution time. A Multi-Objective Adaptive Immune Algorithm (MOAIA) was presented in [102] to solve the ORPD problem. The main idea of the introduced algorithm was to augment two parts to an original immune procedure. The first part marked both 
partial and global facilities to evaluate the antibody affinity to the multi-objective environment. The second part utilized an adaptive crossover, mutation and clone rates, for antibodies to keep the diversifications of the antibodies.

Accordingly, the proposed algorithm managed to establish a dynamic equilibrium between both individual diversity and population convergence.

- Differential Evolution

One of the most popular and efficient EAs to deal with a variety of optimization problems is Differential Evolution (DE) that was first developed by Price and Storn in [103]. In the DE algorithm, the position of each individual is modified according to the difference vector of randomly chosen individuals of the population [103]. Three main operators, namely mutation, crossover, and selection, are incorporated in the DE algorithm. The main tuning parameters, inherent parameters, are only the size of the population, mutation factor, and crossover rate.

A Self-Adaptive DE (SADE) Algorithm for solving the OPF problem in a UPFC equipped power system was proposed in [104], aiming at improving and controlling the power flowing using the UPFC component and under practical security constraints. In addition, new formulae for tuning the algorithm's parameters were designed in such a way that they became automatically adaptive throughout the course of iterations. Furthermore, this work introduced a unique mathematical modeling of the cost function, considering practical security constraints. Multi-Agent-based DE (MADE) Algorithm oriented toward Multi-Agent Systems (MAS) was proposed in [105] for solving the OPF problem considering both non-smooth and non-convex cost functions. Regarding this approach, it should be noted that an agent in the MADE marks a member of the DE algorithm as well as a candidate solution to the current optimization problem in which for obtaining the optimal solution swiftly, each agent competes and cooperates with its counterparts in the close vicinity. A new robust DE algorithm was presented in [106] to solve the SCOPF problem considering a detailed model of the generation unit that includes active/reactive power generation constraints, VPE, multiple fuels, as well as POZs of the units. In addition, security constraints, including restrictions on the voltage amplitude of buses and flow of power in branches in both steady-state and post-contingency states of credible contingencies, were also considered in the proposed problem formulation. A comprehensive optimization algorithm that is oriented to the DE algorithm was successfully evaluated in [107] to solve the constrained version of the OPF problem. In comparison with the available alternatives in the literature, a novel two-stage initialization procedure was proposed in this approach. A Multi-Objective DE (MODE) Algorithm was presented in [108] to minimize the total cost of generation, emission of contaminations, and active power transmission losses in the OPF problem considering FACTS components. Further, a DE-based algorithm was introduced for solving the multi-objective environmental OPF problem in the presence of wind power penetration and shunt-FACTS elements [109]. A fusion optimization algorithm, Sequential Quadratic Programming (SQP) and DE algorithm, was proposed in [110] to solve the OPF problem in which the SQP was implemented to produce individuals, members of an initial swarm, for the DE algorithm that enhanced the efficiency of the resultant algorithm. In another study, the original version of the DE was employed in [111] for solving the OPF problem with different objective functions, including fuel cost, voltage profile, and voltage stability. A robust and efficient DE-based evolutionary algorithm was proposed in [112] for solving the Transient Stability Constrained OPF (TSCOPF) problem. This is a nonlinear optimization problem with both algebraic and differential equations, which is one of the trickiest even in a small power network. In this regard, several policies were suggested for initialization, assessment, and selection processes to decrease the computational burden and improve the efficiency of the resultant algorithm. A modified DE algorithm in order to handle the OPF problem (MDE-OPF) with both non-smooth and non-convex kinds of the fuel cost function was presented in [113], where some modifications in the mutation rule were introduced to improve the convergence rate and also the quality of the obtained optimal solutions. Moreover, the DE algorithm was applied to solve the OPF problem in an equipped power network with FACTS components, TCSC and TCPS elements [114]. A Cooperative Co-Evolutionary DE (CCDE) approach accompanied by a power system decomposition was implemented in [115] to solve the ORPD problem, where a novel decomposition and coordination method was proposed to increase the algorithm's efficiency. The aforementioned method was according to the cooperative co-evolutionary configuration and also the voltage-VAR sensitivity-based power system decomposition technique. In another study in the realm of solving the ORPD problem, a DE algorithm was successfully implemented to solve the problem and improve the controllability of voltage profile in power systems [116] in which the problem was formulated as a mixed-integer nonlinear programming problem, which considered both continuous and discrete control variables. Furthermore, operational inequality limitations 
were handled by the "penalty parameter-less" approach that showed a significant improvement in avoiding the time-consuming trial and error processes to fix the penalty parameter.

\section{- $\quad$ Evolutionary Programming}

Evolutionary Programming (EP) was initially proposed by Fogel in 1960 to optimize finite state machines, where the inheritance relationship and behavior between parents and offspring was highlighted [117]. As the EP simulates the evolution at the species level, no crossover operator is employed.

A comparative study of the MOOPF problem based on DE algorithms, including EP, was conducted in [21] where a Self-Adaptive EP (SAEP) Algorithm was presented to solve the multiobjective optimal operation of the power system [118]. This approach was based on a combination of general EP and random search techniques that involved a self-adaptive mutation operator. An SAEP algorithm was employed to solve the OPF integrated with wind power generators in [119], considering small signal stability constraints. In another study, an EP-based OPF was introduced in [120]. Based on the author's discussion, classical methods are highly sensitive to starting points in optimization problems with a non-monotonic solution surface; consequently, they frequently converge to an optimal local solution rather than a very high-quality solution (or the global one) or even diverge altogether. Therefore, it will be much better to solve these kinds of problems with an EP algorithm. Further, an efficient and reliable EP algorithm was introduced in [121] for solving the OPF problem. The proposed algorithm managed to solve the OPF problem properly regardless of the class of cost function. In addition, to improve the convergence speed of the resultant algorithm and its ability to handle large-scale systems, the algorithm was equipped with gradient information.

- $\quad$ Tabu Search

Tabu Search (TS), a meta-heuristic optimization algorithm, was originally introduced by Glover [122]. The main characteristic of the TS is the use of an adaptive memory accompanied by a responsive exploration. In this connection, a simple model of the TS algorithm combines a local search procedure and anti-cycling memory-based rules to preclude the search from becoming trapped in undesirable local optima. In particular, TS has the ability to avoid reverting to recently explored regions by making a list, namely Tabu List. In addition, TS produces many trial solutions in the vicinity of the current solution and chooses the best one. It is notable that the best trial solution among the generated solutions becomes a current solution.

An efficient and reliable TS-based approach was proposed in [123] for solving the OPF problem. One of the main merits of the proposed approach was conspicuous robustness to its own parameter settings as well as the initial solution.

\section{- $\quad$ Group Search Optimizer}

Inspired by animal searching behavior, the Group Search Optimizer (GSO) is a framework based on the producer-scrounger model, which supposes that the individuals of a group make a quest either for "finding" (producer) or for "joining" (scrounger) opportunities. Therefore, each member in a GSO algorithm is considered as a producer, scrounger, or ranger according to the numerical value of its fitness function, each of which is elaborated upon hereunder [124].

A producer is the member with the best fitness among all individuals in the current generation, which leads the direction of searching for the next generation. Scroungers are usually $80 \%$ of the remaining individuals, which are fortuitously chosen as scroungers to follow the producer to join its resource. Rangers are the rest part in the GSO algorithm, which are responsible for discovering distributed resources in the search space by randomly moving.

An Adaptive GSO (AGSO) Algorithm with improved convergence characteristics was proposed in [125] to solve the OPF problem where different dimensions of the OPF problem were studied to make a precise multi-objective model. In another study, an Improved GSO (IGSO) Algorithm was introduced in [126] to solve the discontinuous non-convex transient stability constrained OPF problem. This algorithm used a backward searching strategy, namely, the Cauchy mutation and inheritance operator, and thus was tailored to cope with the challenges arisen by discontinuous and non-convex properties.

\section{- Biogeography-Based Optimization}

The Biogeography-Based Optimization (BBO) Algorithm that was developed by Simon in [127] functions according to the theory of biogeography, and the concept behind is mainly based on migration and mutation. 
An Adaptive Real Coded BBO (ARCBBO) Algorithm was proposed in [128] to solve the OPF problem in a deregulated power system where different objective functions, including fuel cost, voltage profile, and voltage stability, were studied thoroughly. A new version of the BBO algorithm with an adaptive mutation scheme and the concept of predator-prey optimization technique-an enhanced version of the original $\mathrm{BBO}$ with much better exploitation and exploration capabilitieswas introduced in [129] for solving the MOOPF problem in which the predators carry out a big search to find the best prey in a concentrated manner, while the prey explore the solution space to stay away from the predators. A BBO algorithm was implemented in [130] to solve the multi-constraint OPF problem by considering emission and non-smooth cost function, different constraints.

- $\quad$ Charged System Search Algorithm

Charged System Search Algorithm (CSSA) is another population-based optimization algorithm initially introduced by Kaveh and Talatahari in 2010 in [131] whose mechanism is oriented toward Coulomb Gauss laws.

An Enhanced CSSA (ECSSA) was presented in [132] to solve the reserve constrained DOPF problem subject to VPE, POZs, as well as MFO. The proposed algorithm was equipped with a novel mutation operator to improve the diversity of the initial population and also the convergence characteristics.

- Invasive Weed Optimization

Invasive Weed Optimization (IWO) is also a population-based optimization algorithm that was proposed by Mehrabian and Locus [133].

A Chaotic IWO (CIWO) Algorithm was proposed to solve the nonlinear, non-smooth, and non-convex versions of the OPF problem in [134], in which the proposed approach managed to obtain high-quality solutions for each one of these cost functions.

- $\quad$ Backtracking Search Algorithm

Backtracking Search Algorithm (BSA), a relatively new evolutionary algorithm, was proposed by Pinar Civicioglu for the first time, which uses selection, mutation, and crossover operators such as the DE algorithm [135].

In this regard, the BSA was successfully implemented in [136] to solve the OPF with VPE and POZs. In order to authenticate the ability of the proposed approach in finding a very high-quality solution, it was applied to four different case studies. It was observed that the BSA could reach a very high-quality solution quickly.

- $\quad$ Symbiotic Organisms Search

Inspired by the symbiotic interactions observed between two organisms in the ecosystem, Symbiotic Organisms Search (SOS) is a population-based algorithm initially developed by Cheng and Prayogo [137]. This algorithm privileges two phases, including mutualism and commensalism, for solving any types of optimization problems, and does not require any procedure to specify its control parameters.

The SOS algorithm was applied to solve the OPF problem in a FACTS devices-equipped power system where different objective functions, fuel cost, transmission active power loss, and emission were considered [138]. Further, two different test systems with different scales were employed to demonstrate the ability of the proposed approach.

- $\quad$ League Championship Algorithm

League Championship Algorithm (LCA), a population-based optimization algorithm inspired by the competition of the sports teams that provides an artificial sports league for several weeks, iteration, and over a number of seasons, was developed by Husseinzadeh-Kashan for the first time [139]. Each team, individual in the algorithm, in the league that is defined as a population represents a feasible solution to the given optimization problem to be solved [140].

The aforementioned LCA was proposed in [141] to solve the OPF problem with different objective functions in the Algerian power grid in which a cogent comparison with the available alternatives in the literature was conducted in terms of the effectiveness of the obtained results.

- Clonal Selection Algorithm

Like most of the previous ones inspired by the natural mechanisms in the real-world clonal selection, Clonal Selection Algorithms (CSAs) are a class of optimization algorithms oriented to the clonal theory of acquired immunity that explains how A and B lymphocytes improve their response to antigens over time, called affinity maturation, which focuses on the Darwinian attributes of the theory [142]. 
An Adaptive Clonal Selection Algorithm (ACSA) was presented in [143] for solving the MOOPF problem considering multi-type FACTS devices-UPFC, IPFC, and GUPFC - and load uncertainty. Different objective functions, total generation cost, transmission losses, and voltage stability index (L-index), were considered as well.

- $\quad$ Flower Pollination Algorithm

Flower Pollination Algorithm (FPA) is also a relatively new meta-heuristic optimization algorithm that is based on the nature pollination process of flowers, which was initially introduced by Yang [144].

Modified FPA (MFPA) was successfully implemented in [145] to solve the practical constraint OPF problem with total cost objective function and provide a comparative study with other heuristic algorithms.

- Jaya Algorithm

Jaya is a novel population-based optimization algorithm that was introduced by Rao in 2016 [146] It consists of only two controlling parameters, i.e., the size of the population and the maximum number of generations.

In the realm of OPF studies, the Jaya algorithm was suggested in [147] to solve the OPF problem with three different objective functions, including generation cost, active power losses, and voltage stability, which are almost always in stark contrast to one another. In addition, the impact of Distributed Generation (DG) resources was scrutinized in detail in the aforementioned study, where DG allocation was executed by a sensitivity-based approach.

\section{- $\quad$ Grey Wolf Optimizer}

Grey Wolf Optimizer (GWO) is a novel, powerful swarm-based and meta-heuristic optimization algorithm that was initially introduced by Mirjalili et al. [148], which is based on the leadership hierarchy of a category of grey wolves while they are hunting a prey [149].

A GWO was proposed in [150] to solve the single and multi-objective versions of the OPF problem in detail in which objective functions were minimizing the total generation cost in the presence of VPE, minimizing the total active and reactive power losses of the network, and finally improving the Voltage Security Index (VSI).

\section{- Whale Optimization Algorithm}

Like the GWO, Whale Optimization Algorithm (WOA) is a relatively new optimization algorithm based on a bubble-net hunting strategy (hunting behavior of humpback whales), which was also introduced by Mirjalili in 2015 [151].

Accordingly, WOA was introduced in [152] to solve a multi-objective OPF problem with different objective functions, including total fuel cost, active power losses, and reactive power losses, which are not in accord with one another. Therefore, the optimization problem might be kind of tricky. In this connection, the obtained optimal results were compared with those available in the literature to corroborate the ability of the proposed WOA in recognizing a much better optimal solution for the OPF problem.

- Hybrid Algorithms

Recently, the hybridization of EAs has been becoming popular due to their capabilities in handling optimization problems with high complexity, noisy environment, imprecision, and uncertainty. Hybrid EAs profit from the merits of different EAs and that is why they have gotten popular for solving a variety of complex optimization problems [153].

To date, various hybrid configurations with different mechanisms have been introduced to solve the different versions of the OPF and the ORPD problems. In this connection, the most conspicuous ones of which are elaborated upon hereunder. A Multi-Objective Hybrid Evolutionary Strategy (MOHES) was presented in [154] for solving a comprehensive model of the OPF problem considering total generation cost, emission, active power transmission losses, and voltage deviation. The proposed OPF formulation was organized as both single- and multi-objective optimization problems. A Hybrid TS-SA approach was proposed in [155] to solve the OPF problem in the presence of FACTS devices. From FACTS devices, TCSC and TCPS were integrated in the OPF formulation. A combination of the Improved EP (IEP) and non-linear Interior Point (IP) method was introduced in [156] to solve the ORPD problem. In addition, a hybrid DE-EP algorithm (DEEP) was proposed in [157] for solving the ORPD problem in four different power systems. A powerful hybrid optimization algorithm based on SFLA and SA algorithms was introduced in [10] for solving the non-smooth and non-convex models 
of the OPF problem considering VPE, POZs, and some other practical constraints. A combination of $\mathrm{DE}$ and $\mathrm{ABC}$ algorithms, namely DE-ABC, was implemented in [158] for solving the ORPD problem, whose objective function was active power transmission losses. A hybrid evolutionary algorithm based on harmony search and ant algorithm was proposed in [159] to solve the OPF problem in a FACTS devices-equipped power system in which three different and conflicting objective functions including active power losses, index of active power flow, and voltage deviation were investigated in detail.

\section{Evaluation of the Research Works from Various Standpoints}

All of the reviewed aforementioned references are classified into different categories from various standpoints in this section to determine the fastest way to find a specific paper.

To begin with, Table 2 provides a classification from the optimization algorithm stance in which all of the OPF studies are categorized according to implemented solvers (i.e., optimization algorithms). From this table, it is inferable that the hybrid optimization algorithms are trending, which can be due to the following reasons: (1) they can be simply implemented to solve a variety of optimization problems, (2) they have a combination of the strong points associated with at least two separate optimization algorithms, and (3) they can significantly reduce the computational complexity of the OPF problem. However, the aforementioned advantages are highly dependent on the hybridizing approaches based on which more than one optimization algorithms are combined. As a case in point, three different algorithms were first modified via a self-adaptive mutation operator and then hybridized in a parallel fashion to solve a transmission expansion planning problem integrated with wind farms [10]. The developed hybrid algorithm in [10] resulted in zero standard deviation, meaning that the algorithm converged to the same optimal solution even after 30 independent trials, which is a stupendous achievement in the field of heuristic algorithms.

Table 2. Classification of references based on optimization algorithms.

\begin{tabular}{|c|c|}
\hline Optimization Algorithm & Ref. \\
\hline GA & {$[17,19-26,30]$} \\
\hline PSO & {$[1,12,21,28-33,35]$} \\
\hline HBMO & {$[36,47]$} \\
\hline $\mathrm{ABC}$ & {$[37-46,48]$} \\
\hline SFLA & {$[15,50-52]$} \\
\hline BAT & [55] \\
\hline FA or FFA & {$[57,58]$} \\
\hline GSA & [61-65] \\
\hline ICA & {$[67,69,70]$} \\
\hline $\mathrm{CS}$ or $\mathrm{COA}$ & {$[72-74]$} \\
\hline BFA & [76-80] \\
\hline HSA & {$[82-84]$} \\
\hline $\mathrm{ACO}$ & [86] \\
\hline SA & [88] \\
\hline BHA & {$[91,92]$} \\
\hline TLBO & [94-100] \\
\hline AIS & {$[102]$} \\
\hline $\mathrm{DE}$ & [104-106,108,109,111-116] \\
\hline $\mathrm{EP}$ & {$[118-121]$} \\
\hline TS & [123] \\
\hline GSO & {$[125,126]$} \\
\hline $\mathrm{BBO}$ & [128-130] \\
\hline CSSA & [132] \\
\hline IWO & [134] \\
\hline BSA & [136] \\
\hline SOS & [138] \\
\hline LCA & [141] \\
\hline CSA & [143] \\
\hline FPA & [145] \\
\hline Jaya & [147] \\
\hline GWO & {$[149,150]$} \\
\hline WOA & [152] \\
\hline Hybrid & {$[10,11,15,19,33,53,59,60,68,110,153-159]$} \\
\hline
\end{tabular}

Another compelling classification of references can be based on their under-studied objective functions. In this connection, in order to compare obtained results, researchers tend to look for congruous papers that have considered the same objective function. Therefore, the best shortcut 
to find such resources can be a comprehensive review paper that classifies different studies based on their objective functions. To this end, all papers in the avenue of solving the OPF and ORPD problems are categorized hereunder based on their objective functions, which Table 3 tabulates this comparison. According to this table, one can infer that (1) total generation cost and power loss are the most conspicuous objective functions in the OPF studies and (2) ramp rate (RR) constraint is barely considered in the OPF problem formulation, which can be a good candidate to be considered in the OPF problem for the future works.

Table 3. Classification of references based on objective functions and practical limitations as well.

\begin{tabular}{cc}
\hline Objective and Constraints & Ref. \\
\hline Objective functions & {$[1,12,13,15,17-25,28-30,33-46,50-52,57-59,61-65,67-$} \\
& $70,72,73,76-80,82,83,86,88,91,92,94-99,104-114,118-$ \\
Total generation cost & $123,125,126,128-$ \\
& $130,132,134,136,138,141,143,145,147,150,152,154,155]$ \\
Emission & {$[15,21,29,33,37,38,43,44,50,61,65,69,95-$} \\
& $97,108,109,118,125,128,130,138,141,154]$ \\
Losses of power & {$[15,16,18,19,21,26,29,31-33,37,38,43,44,47,48,51-$} \\
& $53,55,57,59,61,63-65,68-70,73,74,79,83,84,94,95,97-$ \\
Voltage deviation & $100,108,110,115,116,118,128-$ \\
& $130,138,141,143,145,147,149,150,152-154,156-159]$ \\
Voltage stability & {$[28,31,37,38,47,52,57,59,61,63,68-$} \\
Practical constraints & $70,74,84,94,95,98,100,102,128,129,141,145,149,153]$ \\
& {$[18,19,21,22,28,38,41,47,51,61,73,79,83,84,97-$} \\
VPE & $100,102,125,128,129,141,143,147,150]$ \\
POZ & {$[1,11,15,18,29,33,35-38,40,42-44,46,61-63,65,68,72,82,95-$} \\
MFO & $97,108-$ \\
RR & $110,113,114,123,126,130,132,134,136,138,141,145,150,154]$ \\
& {$[1,15,35,38,40,42,61,62,68,72,91,95,98,111,113,132,134]$} \\
& {$[11,36]$} \\
\hline
\end{tabular}

It is also worth stating that some test systems are well known for OPF and ORPD studies. Therefore, a classification based on under-studied test systems can be a valuable tool to help researchers to find their desired paper. To this end, Table 4 categorizes all test systems implemented in the realm of handling the OPF and ORPD problems.

Table 4. Classification of references based on test systems.

\begin{tabular}{|c|c|}
\hline Test System & Ref. \\
\hline IEEE 5-bus & {$[39,52,55]$} \\
\hline IEEE 6-bus & {$[82,105,113,118]$} \\
\hline IEEE 9-bus & {$[38,39,42,130]$} \\
\hline IEEE 14-bus & {$[26,36,59,82,104,107,116,157,158]$} \\
\hline IEEE 26-bus & {$[17,42,63,106]$} \\
\hline IEEE 30-bus & $\begin{array}{c}{[1,12,15-23,26,28-31,33-48,50-53,55,57-62,68,69,72-74,76-78,80,82-} \\
84,86,88,92,95-100,102,104-106,108- \\
111,113,114,116,118,120,121,123,125,128- \\
130,132,134,136,138,143,145,150,152,154,155,157-159]\end{array}$ \\
\hline IEEE 57-bus & $\begin{array}{c}{[15,29,35,38,47,53,62,65,68-} \\
70,72,82,84,95,96,104,108,116,125,128,138,153,157,158]\end{array}$ \\
\hline IEEE 118-bus & $\begin{array}{c}{[15,17,18,23,31-33,36,37,42,46-48,53,60,63,72,76,82,91,97-} \\
101,104,106,110,115,116,132,147,150,153,154,156,157]\end{array}$ \\
\hline IEEE 145-bus & {$[126]$} \\
\hline IEEE 300-bus & {$[18,42]$} \\
\hline IEEE 354-bus & [31] \\
\hline WSCC 9-bus & [126] \\
\hline New England 39-bus & {$[26,45,79,106,112,119,126]$} \\
\hline Algerian 59-bus & {$[92,141]$} \\
\hline Indian 62-bus & [97] \\
\hline 17 unit $162-$ bus & [112] \\
\hline
\end{tabular}


From Table 4, it is clear that among all of the tabulated test systems, there are three power networks that are appealing to many researchers, which include IEEE 30-, 57-, and 118-bus test systems. In this connection, in order to provide more clarity, the corresponding data associated with IEEE 30-, 57-, and 118-bus test systems are, respectively, provided in Appendix A, Appendix B, Appendix C. Moreover, the IEEE 30-bus test system has received a lot of attention for OPF studies compared to the other two networks, i.e., the IEEE 57- and 118-bus systems, since researchers can investigate different versions of the OPF problem on this test system, such as OPF considering multiple energy carriers, valve point effect, prohibited operating zones, etc. (see Appendix A).

Elementally, solving the optimization problems in general and miscellaneous models of OPF problems can be divided into two completely different kinds, including single- and multi-objective methodologies. In this regard, it is worth stating that there are two major, different approaches to solve the OPF problem as a Multi-Objective Optimization Problem (MOOP), including penalty and Pareto optimal methods, the merits and demerits of which are elaborated upon hereunder.

- $\quad$ Penalty method: There are some advantages to penalty methods. For example, it is very simple and effective since they do not need a Pareto ranking routine. However, there are disadvantages, as well. For instance, it may be tough to recognize suitable coefficients. This issue becomes bold when there is not enough information about the problem to be optimized, which is a crucial concern, especially in dealing with real circumstances [128,129].

- Pareto optimal method: There are many merits to the Pareto optimal method. For example, power system operators can readily obtain their desirable trade-offs between different objective functions by adopting weight factors. There are some drawbacks as well, such as increasing the execution time and requiring a powerful optimizer to handle the problem and find welldistributed Pareto fronts [160-165].

Accordingly, with all this taken into account, Table 5 classifies all references based on their implemented optimization methodologies in order to have a comprehensive view. From this table, one can infer that different types of multi-objective approaches in the field of OPF problems have received lower attention compared to the single-objective frameworks, which is due to the fact that a multi-objective OPF problem is oriented toward optimizing more than one objective function at the same time; hence, such problems impose more complications to the OPF problem compared to single-objective OPF problems. Moreover, it is challenging for optimization algorithms to find a suitable trade-off between different objective functions, which may not be in accord with each other.

Table 5. Classification of references based on optimization methodologies.

\begin{tabular}{cc}
\hline Optimization Methodology & Ref. \\
\hline Single-objective & {$[1,15-24,26,28-48,50-53,55,57-65,67-70,72-$} \\
& $74,76-80,82-84,86,88,91,92,94-$ \\
& $101,104,105,116,118-123,125,126,128-130,132$, \\
Multi-objective by penalty method & $134,136,138,141,143,145,147,149,150,152-159]$ \\
Multi-objective by Pareto optimal method & {$[25,29,63,64,68,70,76,79,95,98,100,102,128-$} \\
Multi-objective by SPEA & $130,141,143,149]$ \\
Multi-objective by Fuzzy techniques & {$[15,16,21,22,43,44,47,50,51,59,61,69,83,94,96,97$,} \\
& $99,108,109,125,150,154]$ \\
& {$[15,19,118]$} \\
\end{tabular}

Inasmuch as loads of the system increase rapidly, controlling the transmission network has been much trickier in modern-day power systems. In this regard, the application of Flexible Alternating Current Transmission Systems (FACTS) devices is one of the most appropriate and apt alternatives to prevent such aforementioned issues. Thanks to enjoying the appropriate power electronic technologies, FACTS devices have key roles in power systems. To name but a few, reducing the generation costs, reducing the power losses, improving the voltage stability, and increasing the security of the power system are the most conspicuous ones. Table 6 categorizes all affected references utilizing different forms of the FACTS devices and scrutinizing the OPF problem with and without considering FACTS components. 
Table 6. Classification of references based on implementing FACTS devices.

\begin{tabular}{cc}
\hline FACTS Device & Ref. \\
\hline High Voltage Direct Current (HVDC) & {$[26]$} \\
Static VAR compensator (SVCs) & {$[17,23,51,59,78,159]$} \\
Static compensator (STATCOM) & {$[80,109]$} \\
Thyristor-controlled series compensator & (TCSC) \\
Thyristor-controlled phase shifter transformer & {$[1,51,52,65,78,114,138,155,159]$} \\
(TCPST) & {$[24,65,114,138,155,159]$} \\
Thyristor-controlled voltage regulator (TCVR) & {$[159]$} \\
Unified power flow controller (UPFC) & {$[15,52,55,58,79,104,143]$} \\
Interline power flow controller (IPFC) & {$[143]$} \\
Generalized unified power flow controller & \\
(GUPFC) &
\end{tabular}

Utilizing different types of FACTS devices can be considered a double-edged sword, and their merits and demerits are inextricably interconnected. For instance, power system operators need to determine the optimal location of FACTS components in order to improve the controllability of the power grid; however, as long as they are optimally allocated, they have a drastic impact on the operation of power systems, e.g., reducing the power loss and improving the voltage profile, to name but two, by providing rapid responses. On the flip side, FACTSs are expensive, which makes them unreasonable to be utilized for the sole purpose of power system stability enhancement. Thus, it is crucial to find a trade-off between the aforementioned aspects of the FACTS components.

Last but certainly not least, hastening the power industry reregulation juxtaposed with the unrivaled implementation of uncertain renewable resources as well as smart grid utilization faces power system operation with severe uncertainties. Therefore, harnessing the uncertainties in power system optimization problems integrated with renewable resources is an obligation [166,167].

Renewable energy resources have been employed in different parts of power systems [168-170]. However, classifying OPF and ORPD problems from the renewable energy implementation point of view is less studied. To this end, OPF and ORPD studies integrated with renewable energy resources are classified in Table 7. It should be noted that photovoltaic (PV) and wind resources have been the most utilized resources in this regard; therefore, the proposed classification has been established based on these resources [171-177]. Nevertheless, the biggest challenge for integrating PV modules and wind turbines into the power grids is their intermittent nature; therefore, the generated power from these resources are uncertain, meaning that in some time intervals, the output power might be more than or less than the scheduled power, which may lead to over/underestimation of the available level of electrical energy in the system. As a result, the power system operator should be ready to address the aforementioned challenges upon occurrence. In this connection, providing the way to migrate from deterministic to stochastic approaches, probabilistic analysis is gaining more attention in providing robust optimal solutions [91]. One common method to approach the problem is scenario-based stochastic programming handling the uncertainties associated with different power sources and system's demand in the OPF problem. Hence, the uncertain optimization problem is turned into an equivalent number of deterministic problems by providing various scenarios via roulette wheel mechanism, for instance. It is, therefore, inferable that the higher the number of scenarios, the higher the accuracy; however, a large number of scenarios may result in computational inefficiency. Hence, it is essential to reduce the number of scenarios by removing the less important ones through scenario reduction methods [178].

Table 7. Classification of references based on the renewable energy resources used in them.

\begin{tabular}{cc}
\hline Renewable Energy Resources & Ref. \\
\hline Wind power & {$[33,34,39,40,80,91,119,171-175]$} \\
Photovoltaic & {$[91,171,173,176,177]$} \\
\hline
\end{tabular}

Recently, power system restructuring accompanied by introducing a new generation of renewable energy sources into the power industry have transformed the traditional, passive distribution networks into real-time, active systems, which can be a target of different kinds of cyberattacks, above all false data injection (FDI) attacks [12]. For example, in [12], the OPF problem was approached by a 
hacker to generate a model of FDI cyberattack leading to (1) system congestions and (2) higher value of voltage profile. Interested readers are directed to [179-181] for more information (i.e., frameworks and formulations) about cyberattacks targeting smart transmission and distribution systems. Finally, Table 8 presents a comprehensive review of the most recent advancement in the field of cybersecurity, i.e., false data injection (FDI) attack model and remedial action scheme (RAS) to mitigate the negative impacts of cyberattacks in modern power systems.

Table 8. Overview of the recent publications in the field of cybersecurity.

\begin{tabular}{|c|c|c|c|}
\hline \multirow{2}{*}{ Ref. } & \multicolumn{2}{|c|}{ Framework } & \multirow{2}{*}{ Objective } \\
\hline & FDI & RAS & \\
\hline [182] & $\checkmark$ & & $\begin{array}{c}\text { Coordination between load redistribution (LR) attack and } \\
\text { current-carrying elements (i.e., lines and generators) to show the } \\
\text { potential damaging impacts of the coordinated attacks on IEEE 14-bus } \\
\text { test system }\end{array}$ \\
\hline$[183,184]$ & $\checkmark$ & & $\begin{array}{l}\text { Injecting arbitrary errors into certain state variables by FDIs against } \\
\text { state estimation, which bypass the bad data detection }\end{array}$ \\
\hline [185] & $\checkmark$ & & $\begin{array}{l}\text { Formulizing the economic impacts of FDIs on IEEE 14-bus test system } \\
\text { in real-time market operation }\end{array}$ \\
\hline [186] & $\checkmark$ & & $\begin{array}{c}\text { Targeting a single day of the (1) Australian electricity market trading } \\
\text { mechanism and (2) IEEE 118-bus test system using an extended FDI } \\
\text { cyberattack }\end{array}$ \\
\hline [187] & $\checkmark$ & $\checkmark$ & $\begin{array}{l}\text { Changing the price of electricity in a desirable direction as well as } \\
\text { defending the system to react to FDIs on PJM 5-bus test system }\end{array}$ \\
\hline [188] & $\checkmark$ & & $\begin{array}{l}\text { Making cascade outages as well as imposing large damages into the } \\
\qquad 118 \text {-bus test system }\end{array}$ \\
\hline [189] & $\checkmark$ & & $\begin{array}{l}\text { Injecting false data into the process of security-constrained economic } \\
\text { dispatch (as a trilevel model) on IEEE 14-bus test system }\end{array}$ \\
\hline [190] & $\checkmark$ & & $\begin{array}{c}\text { Targeting multiple tie-lines at the same time in economic dispatch } \\
\text { problem (as a bilevel FDI and a mixed-integer linear programming } \\
\text { problem) on IEEE 118-bus test system }\end{array}$ \\
\hline [191] & $\checkmark$ & $\checkmark$ & $\begin{array}{l}\text { - Manipulating the state variables based on only the data } \\
\text { associated with the susceptance of tie-lines } \\
\text { Presenting suitable countermeasures to keep all buses hidden to } \\
\text { the attackers on IEEE 9-, 14, 30, and 118-bus test systems }\end{array}$ \\
\hline [192] & & $\checkmark$ & $\begin{array}{l}\text { Reacting (in the form of real-time smart distribution network } \\
\text { reconfiguration) to the detected FDIs over retailers in the electricity } \\
\text { market (distributed generation retailers as well as electric vehicle and } \\
\text { demand response retailers) on the 136-bus distribution system }\end{array}$ \\
\hline [193] & & $\checkmark$ & $\begin{array}{l}\text { Recovering the manipulated data on IEEE } 30 \text { - and 118-bus test systems } \\
\text { using a defense mechanism based on a statistical physical model and a } \\
\text { generative adversarial network-based cyber model simultaneously }\end{array}$ \\
\hline [194] & & $\checkmark$ & $\begin{array}{c}\text { Responding to the FDIs expeditiously by re-dispatching the generation } \\
\text { units on IEEE 24- and 118-bus test systems using OPF aiming at } \\
\text { optimizing the system security }\end{array}$ \\
\hline [195] & & $\checkmark$ & $\begin{array}{c}\text { Proposing a cyber-secured unit commitment problem that is reliable } \\
\text { against LR attacks on IEEE 118-bus tests system }\end{array}$ \\
\hline
\end{tabular}


Table 8. Cont.

\begin{tabular}{|c|c|c|c|}
\hline \multirow{2}{*}{ Ref. } & \multicolumn{2}{|c|}{ Framework } & \multirow{2}{*}{ Objective } \\
\hline & FDI & RAS & \\
\hline [196] & & $\checkmark$ & $\begin{array}{l}\text { Improving the transient stability of WECC 179-bus system broken into } \\
\text { several islands as a result of FDIs and supporting the islands provide } \\
\text { their services independently }\end{array}$ \\
\hline [197] & & $\checkmark$ & $\begin{array}{c}\text { Scrutinizing the vulnerability of the IEEE 14-bus test system via fault } \\
\text { chain theory as well as proposing a new vulnerability index as a RAS } \\
\text { to diminish the vulnerability and reinforce the system against } \\
\text { future attacks }\end{array}$ \\
\hline [198] & & $\checkmark$ & $\begin{array}{c}\text { Detecting the coordinated FDI attacks as well as performing the best } \\
\text { RAS in a timely manner on IEEE 39-bus test system via a } \\
\text { multi-agent-based system protection and emergency } \\
\text { control mechanism }\end{array}$ \\
\hline [199] & $\checkmark$ & & $\begin{array}{c}\text { Presenting a bilevel FDI framework leading to severe undervoltage in a } \\
\text { distribution system modified by PV modules }\end{array}$ \\
\hline [200] & $\checkmark$ & & $\begin{array}{l}\text { Developing a hardware-in-the-loop (HIL) testbed to scrutinize the } \\
\text { impacts of cyber attacks targetting lab-scale microgrids }\end{array}$ \\
\hline [201] & $\checkmark$ & $\checkmark$ & $\begin{array}{l}\text { Introducing a three-level framework: (1) planning phase, where } \\
\text { strategic location/number of TCSCs were determined, (2) operation } \\
\text { phase, where the enhanced system in the previous phase was the target } \\
\text { of different scenarios of FDI attacks leading to tie-line congestions, and } \\
\text { (3) reaction phase, where power system operator took advantage of the } \\
\text { pre-installed TCSCs to rapidly alleviate the congestions caused by FDIs }\end{array}$ \\
\hline
\end{tabular}

Contrary to most studies, this paper provides a comparison between different methods in optimizing the cost, one of the most important objective functions in the OPF studies, which is from an economic standpoint. As an illustration, a comparison between different EAs for solving the OPF problem in the most popular and conventional test systems, i.e., IEEE 30-, 57-, and 118-bus test systems, are depicted in Figures 9-11, respectively. This is due to the fact that these three test systems have been under the spotlight of researchers for many years and a great deal of attention has been paid to these systems to solve the different versions of the OPF and ORPD problemsTable 4 approves this fact. It should be noted that the reported costs in Figures 9-11 are associated with minimizing objective function (6) considering (7)-(12) as decision variables. Moreover, in the provided comparisons in Figures 9-11, (49)-(53) are considered as equality constraints (i.e., the power balance constraints) and (54)-(61) are considered as inequality constraints. It is noted that constraints (49)-(53) and also (54)-(61) are the basic constraints in any OPF problem.

Referring to Figures 9-11, it is obvious that the IEEE 30-bus test system is more popular than the other IEEE systems in the field of OPF studies. In addition, the IEEE 30-bus test system is usually considered by researchers to examine the performance of their proposed algorithms in solving different versions of the OPF problem (e.g., OPF considering VPE, POZs, MFO, etc.). Moreover, from Figure 9, one can perceive that the hybrid and enhanced version of the optimization algorithms (e.g., $[1,15,19,68])$ could converge to a better optimal solution compared to the original algorithms (e.g., $[86,120])$. Interested readers are directed to Table 2 for more information about the type of optimizers presented in each reference. Therefore, it is vital to improve the performance of such algorithms when it comes to power system operation since power system operators need to be decisive in decision-making; that is to say, power system operators should be able to expeditiously solve the OPF problem and update the schedule of power dispatch while satisfying the constraints, especially power balance. Furthermore, the performance of the same optimization algorithm might be different when it is applied to different optimization problems with qualitative discrepancies. For example, in the field of OPF studies, the objective function (15) is more complicated than the objective function presented in (6); hence, the search space of the optimization problem associated with (15) has much more local optima compared to the search space of (6). From the practical point of view, local optima are low-quality optimal solutions and should be avoided by improving the searching capability of the optimization algorithms (e.g., by implementing fuzzy systems [1], mutation operators [10], and hybrid structures [163,164]). 


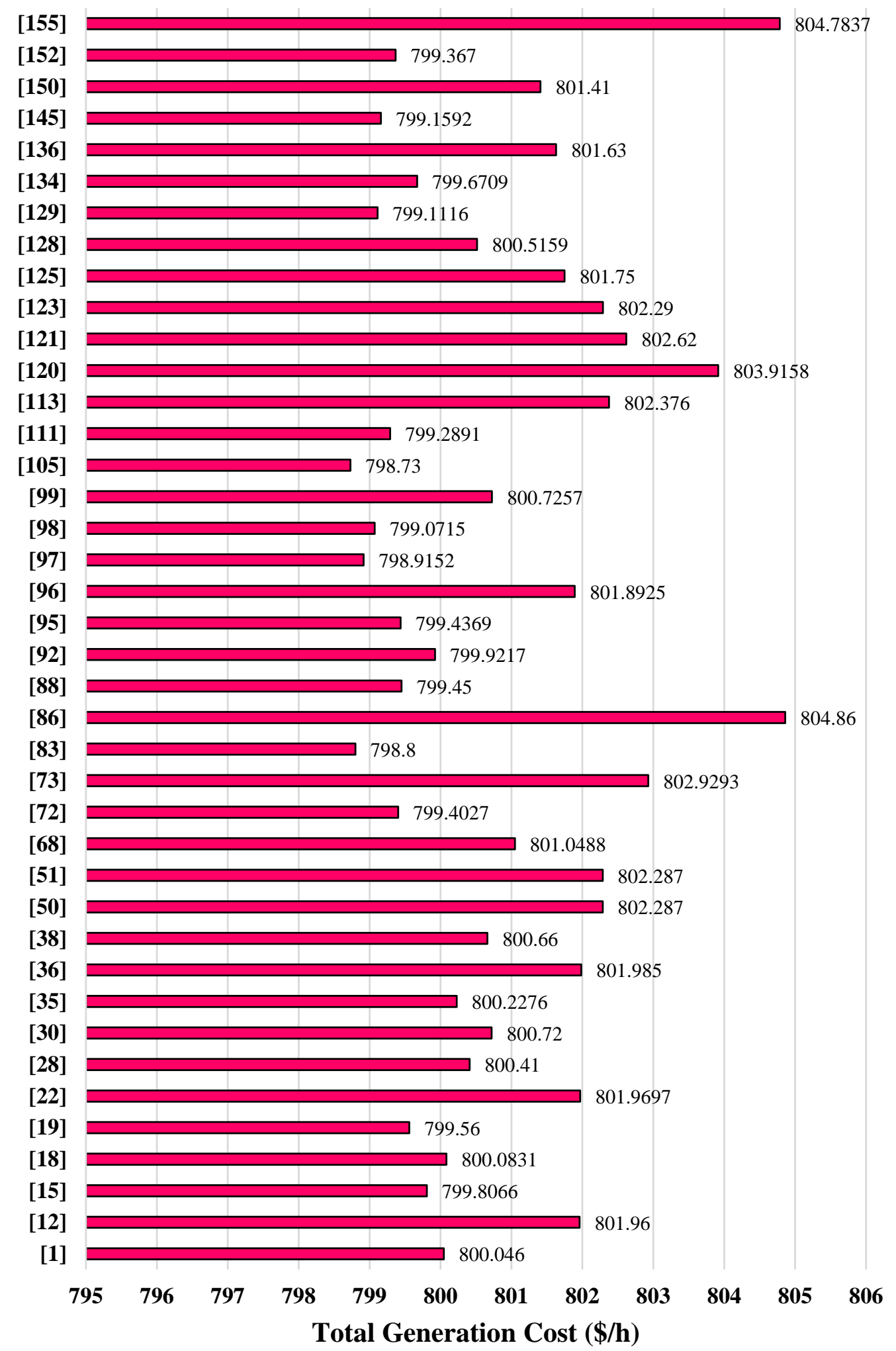

Figure 9. Comparison of cost obtained by different algorithms for IEEE 30-bus test system based on objective function (6). 


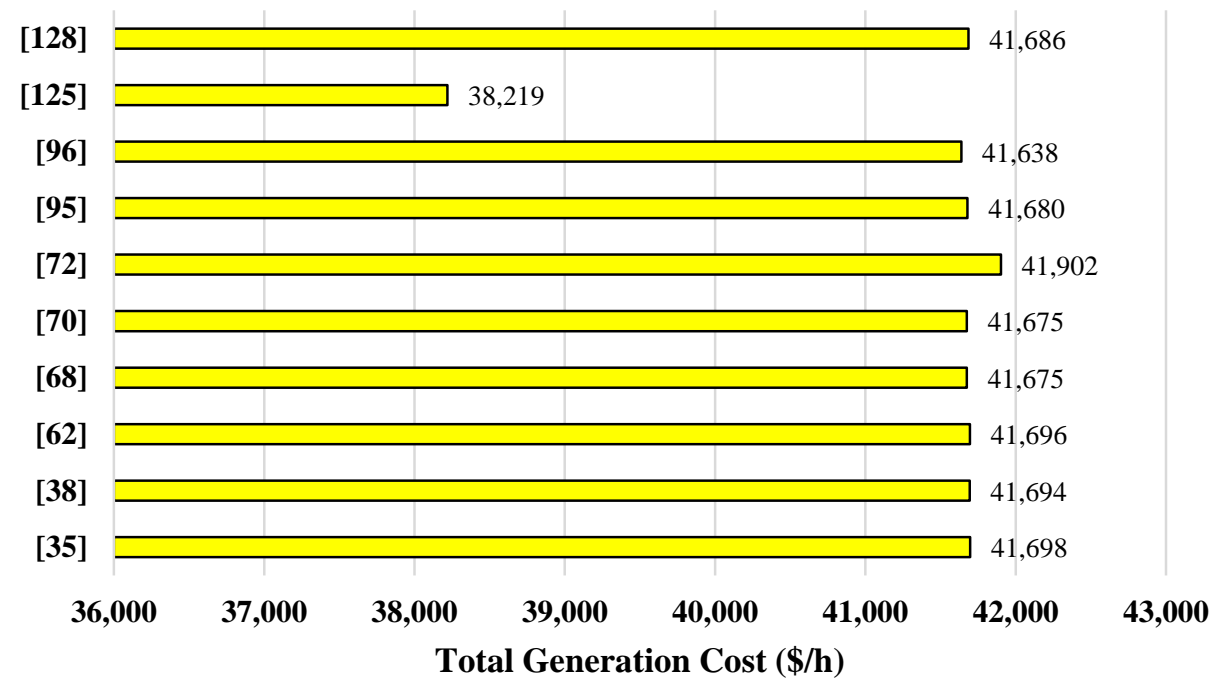

Figure 10. Comparison of cost obtained by different algorithms for IEEE 57-bus test system based on objective function (6).

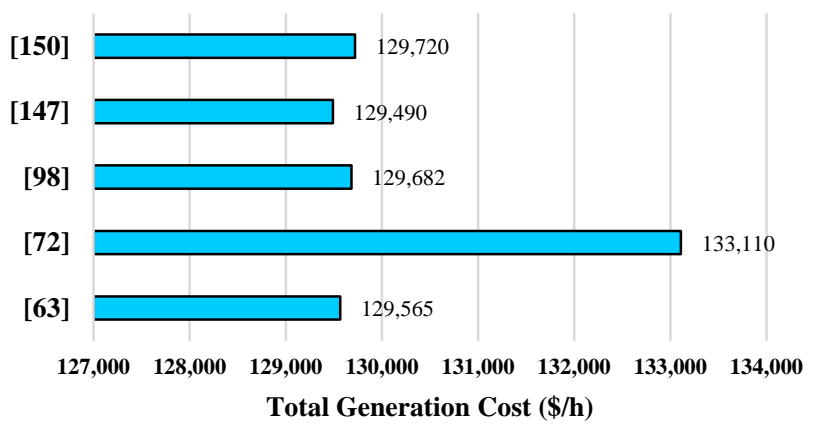

(a) 54-generator

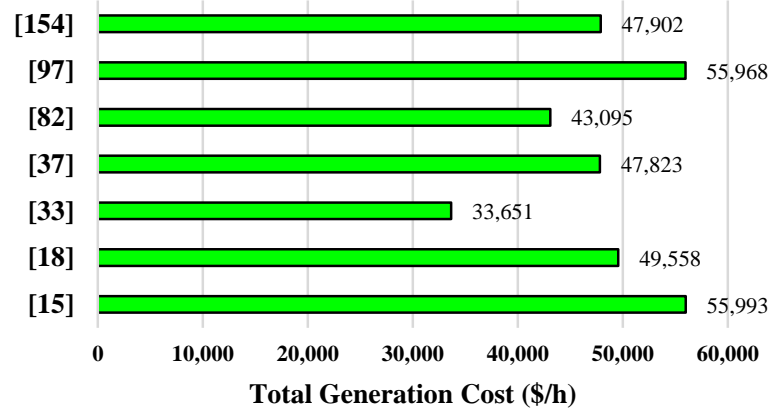

(b) 19-generator

Figure 11. Comparison of cost obtained by different algorithms for IEEE 118-bus test system based on objective function (6): (a) 54-unit test system, (b) 19-unit test system.

\section{Conclusions}

In the literature, the optimal power flow (OPF) problem has been solved using different optimization methods ranging from mathematical to meta-heuristic algorithms. In this connection, heuristic algorithms have numerous merits in solving complicated optimization problems to such an extent that a copious number of these algorithms have been introduced and implemented to solve a variety of OPF problems. In this regard, selection and application of the best algorithm among all presented for a typical problem have been a challenge in the realm of OPF studies because the one that is labeled as a paragon must show a cogent performance in both celerity and preciseness directions. To this end, a comparative analysis is quite a useful and essential reference to find the best algorithm thus far. In this paper, we have tried our best to cover the most popular optimization algorithms utilized in OPF studies, which have been profoundly reviewed and scrutinized from many various standpoints. Accordingly, a broad classification juxtaposed with detailed comparison has been proposed from different aspects, including, as an illustration, optimization algorithms, case studies, objective functions, and constraints, to name but a few. Finally, the presented review article could definitely help researchers be on the right track of understanding new concepts and employing new materials that they might need in dealing with the OPF problem.

Author Contributions: Conceptualization, E.N. and M.P.-K.; methodology, E.N.; validation, E.N., H.N., M.P.-K., and M.L.; formal analysis, F.V.C.; investigation, E.N., M.P.-K., and F.V.C.; resources, E.N. and H.N.; data curation, M.P.-K. and F.V.C.; writing-original draft preparation, E.N.; writing- 
review and editing, M.P.-K. and M.M.; visualization, E.N.; supervision, M.P.-K.; project administration, M.L. All authors have read and agreed to the published version of the manuscript.

Funding: This research received no external funding.

Data Availability Statement: All of the data can be found in the references.

Conflicts of Interest: The authors declare no conflict of interest.

\section{Nomenclature}

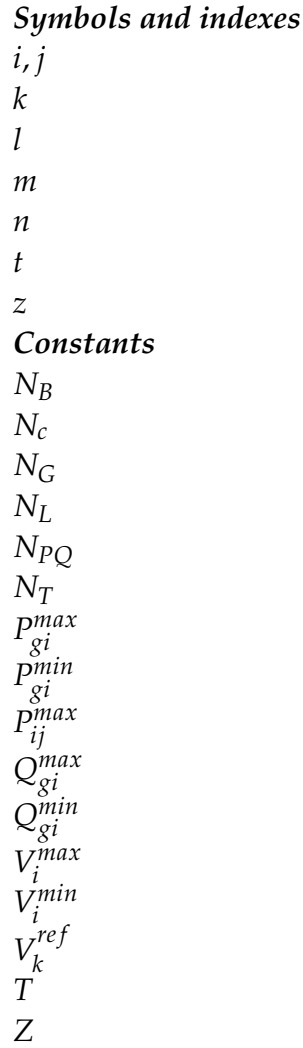

Variables and Parameters $a_{i}, b_{i}, c_{i}, d_{i}$ and $e_{i}$

$C_{M F O}(X)$

$C_{Q}(X)$

$C_{V A R}$

$C_{V A R}^{\text {Fuel }}$

$C_{V A R}^{\text {LOSS }}$

$C_{V P E}(X)$

$D R_{i}$

$E_{L}(X)$

$E_{N L}(X)$

$F_{\text {Loss }}$

$G_{l}$

$L_{j}$

$n$

$P_{g i}$

$P_{g i}^{t}$

$P_{g i}^{(t-1)}$
Controlled buses (PV buses)

Load buses (PQ buses)

Transmission lines

Transformers

Capacitors

Time slot

Prohibited operating zones

Number of the buses

Number of the compensation capacitor

Total number of generators

Number of transmission lines

Number of PQ buses

Number of transformer's tap

Maximum active power value of $i^{\text {th }}$ generator

Minimum active power value of $i^{\text {th }}$ generator

Maximum power flows through the branch

Maximum reactive power value of $i^{\text {th }}$ bus

Minimum reactive power value of $i^{\text {th }}$ bus

Maximum valid voltage for bus $i$

Minimum valid voltage for bus $i$

Voltage magnitude at the $k^{\text {th }}$ load bus, which is usually set to 1.00 p.u.

Total number of intervals

Number of prohibited operating zones

Cost coefficients of the $i^{\text {th }}$ generators

Cost function considering multi-fuel operation in $(\$ / h)$

Quadratic cost function in $(\$ / h)$

Cost of compensator resources

Cost of compensator resources and generation cost

Cost of compensator resources and the cost of active power losses

Cost function considering valve-point effect in $(\$ / h)$

Ramp down bound of $i^{t h}$ generator in $(M W / h)$

Linear model for emission function in $($ ton $/ h)$

Non-linear model for emission function in $($ ton $/ h)$

Real power transmission losses in $M W$

Conductance of the $l^{\text {th }}$ transmission line

The index to measure the static voltage stability margin

Dimension of problem

Output active power of $i^{\text {th }}$ generator

Output active power of $i^{\text {th }}$ unit at $t^{\text {th }}$ time interval

Output power of $i^{\text {th }}$ unit at time $(t-1)$ in $(M W)$ 


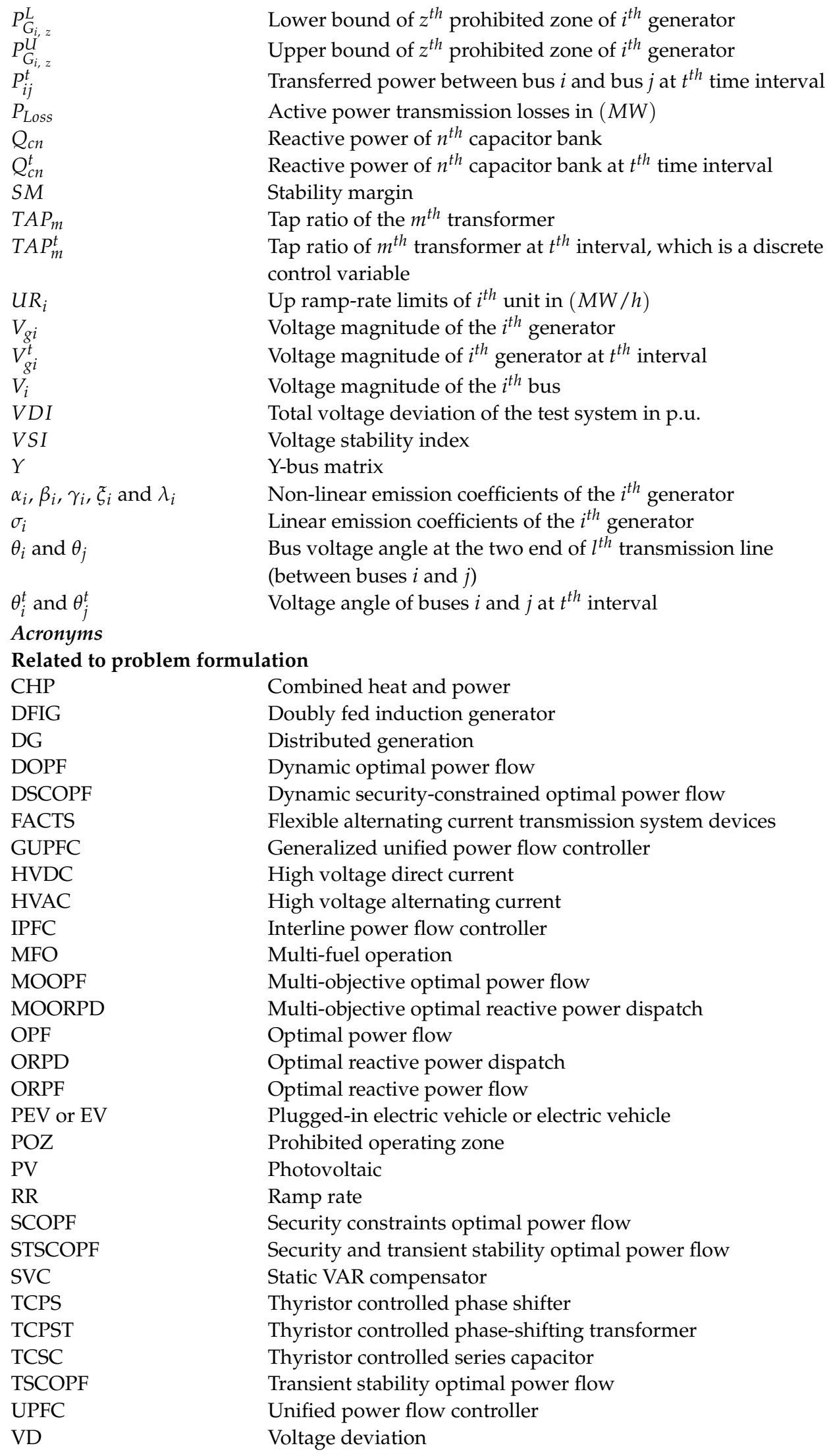




\begin{tabular}{|c|c|}
\hline VDI & Voltage deviation index \\
\hline VPE & Valve-point effect \\
\hline VR & Voltage regulator \\
\hline VSI & Voltage stability index \\
\hline VSL & Voltage stability limit \\
\hline WPP & Wind power plant \\
\hline \multicolumn{2}{|c|}{ Related to optimization algorithms } \\
\hline EAs & Evolutionary Algorithms \\
\hline EEA & Efficient Evolutionary Algorithms \\
\hline GA & Genetic Algorithm \\
\hline EGA & Enhanced Genetic Algorithm \\
\hline EPGA & Efficient Parallel Genetic Algorithm \\
\hline PSO & Particle Swarm Optimization \\
\hline NSGA-II & Non-Dominated Sorting Genetic Algorithm-II \\
\hline ALC-PSO & Aging Leader And Challengers Particle Swarm Optimization \\
\hline EPSO & Evolutionary Particle Swarm Optimization \\
\hline EADPSO & Evolving Ant Direction Particle Swarm Optimization \\
\hline $\mathrm{HBMO}$ & Honey Bee Mating Optimization \\
\hline MABC & Modified Artificial Bee Colony \\
\hline GABC & Gbest Guided Artificial Bee Colony \\
\hline TLBO & Teaching-Learning-Based Optimization \\
\hline EABC & Enhanced Version of Artificial Bee Colony \\
\hline $\mathrm{M}^{2} \mathrm{OBA}$ & Multi-Hive Multi-Objective Bee Algorithm \\
\hline HMOABC & Hybrid Multi-Objective Artificial Bee Colony \\
\hline CABC & Chaotic Artificial Bee Colony \\
\hline QCABC & $\begin{array}{l}\text { Quantum-Theory and the Chaotic-Local Search Strategy of } \\
\text { Artificial Bee Colony }\end{array}$ \\
\hline CPVEIHBMO & $\begin{array}{l}\text { Chaotic Parallel Vector Evaluated Interactive Honey Bee } \\
\text { Mating Optimization }\end{array}$ \\
\hline SFLA & Shuffled Frog Leaping Algorithm \\
\hline MSFLA & Modified Shuffled Frog Leaping Algorithm \\
\hline NM & Nelder-Mead Method \\
\hline FA & Firefly Algorithm (usually known as FFA) \\
\hline EFA & Enhanced Firefly Algorithm \\
\hline FA-PS & Hybrid Firefly Algorithm and Pattern Search \\
\hline HFA & Hybrid Firefly Algorithm \\
\hline GSA & Gravitational Search Algorithm \\
\hline NSMOOGSA & $\begin{array}{l}\text { Non-Dominated Sorting Multi-Objective Opposition-Based } \\
\text { Gravitational Search Algorithm }\end{array}$ \\
\hline OGSA & Opposition-Based Gravitational Search Algorithm \\
\hline ICA & Imperialist Competitive Algorithm \\
\hline ANN & Artificial Neural Network \\
\hline TLA & Teaching-Learning Algorithm \\
\hline MOMICA & Multi-Objective Modified Imperialist Competitive Algorithm \\
\hline MICA & Modified Imperialist Competitive Algorithm \\
\hline CSA & Cuckoo Search Algorithm (usually known as CS Or COA) \\
\hline ICS & Improved Cuckoo Search \\
\hline BFA & Bacteria Foraging Algorithm \\
\hline DBFA & Dynamic Bacteria Foraging Algorithm \\
\hline EBFA & Enhanced Bacteria Foraging Algorithm \\
\hline MBFA & Modified Bacteria Foraging Algorithm \\
\hline HSA & Harmony Search Algorithm (usually known as HS) \\
\hline
\end{tabular}




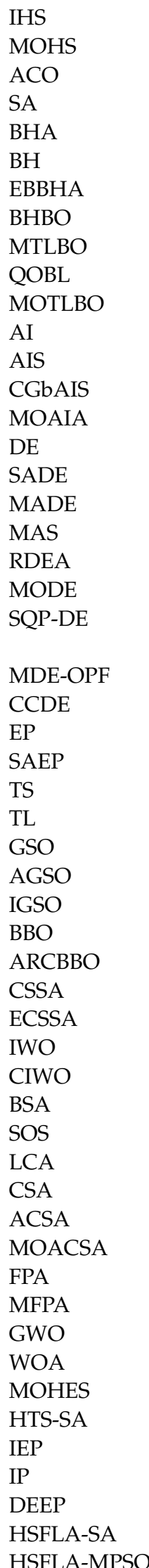

DE-ABC
Improved Harmony Search

Multi-Objective Harmony Search

Ant Colony Optimization

Simulated Annealing

Black Hole Algorithm

Black Hole

Enhanced Binary Black Hole Algorithm

Black Hole-Based Optimization

Modified Teaching-Learning-Based Optimization

Quasi-Oppositional-Based Learning

Multi-Objective Teaching-Learning-Based Optimization

Artificial Intelligence

Artificial Immune Systems (usually known as IS)

Cluster-and-Gradient-Based Artificial Immune Systems

Multi-Objective Adaptive Immune Algorithm

Differential Evolution

Self-Adaptive Differential Evolution

Multi-Agent-Based Differential Evolution

Multi-Agent Systems

Robust Differential Evolution Algorithm

Multi-Objective Differential Evolution

Hybrid Sequential Quadratic Programming and

Differential Evolution

Modified Differential Evolution Algorithm for OPF Problem

Cooperative Co-Evolutionary Differential Evolution

Evolutionary Programming

Self-Adaptive Evolutionary Programming

Tabu Search

Tabu List

Group Search Optimization

Adaptive Group Search Optimization

Improved Group Search Optimization

Biogeography-Based Optimization

Adaptive Real Coded Biogeography-Based Optimization

Charged System Search Algorithm

Enhanced Charged System Search Algorithm

Invasive Weed Optimization

Chaotic Invasive Weed Optimization

Backtracking Search Algorithm

Symbiotic Organisms Search

League Championship Algorithm (usually known as LC)

Clonal Selection Algorithm

Adaptive Clonal Selection Algorithm

Multi-Objective Adaptive Clonal Selection Algorithm

Flower Pollination Algorithm

Modified Flower Pollination Algorithm

Grey Wolf Optimizer

Whale Optimization Algorithm

Multi-Objective Hybrid Evolutionary Strategy

Hybrid Tabu Search and Simulated Annealing

Improved Evolutionary Programming

Interior Point

Hybrid Differential Evolution and Evolutionary Programming

Hybrid Shuffled Frog Leaping Algorithm and Simulated Annealing

Hybrid Shuffled Frog Leaping Algorithm and Modified Particle

Swarm Optimization

Hybrid Differential Evolution and Artificial Bee Colony 


\section{Appendix A Data for Test System 1 (IEEE 30-bus Test System)}

The single-line diagram of the IEEE 30-bus test system is portrayed in Figure A1, and its essential data are utterly tabulated in Tables A1-A5.

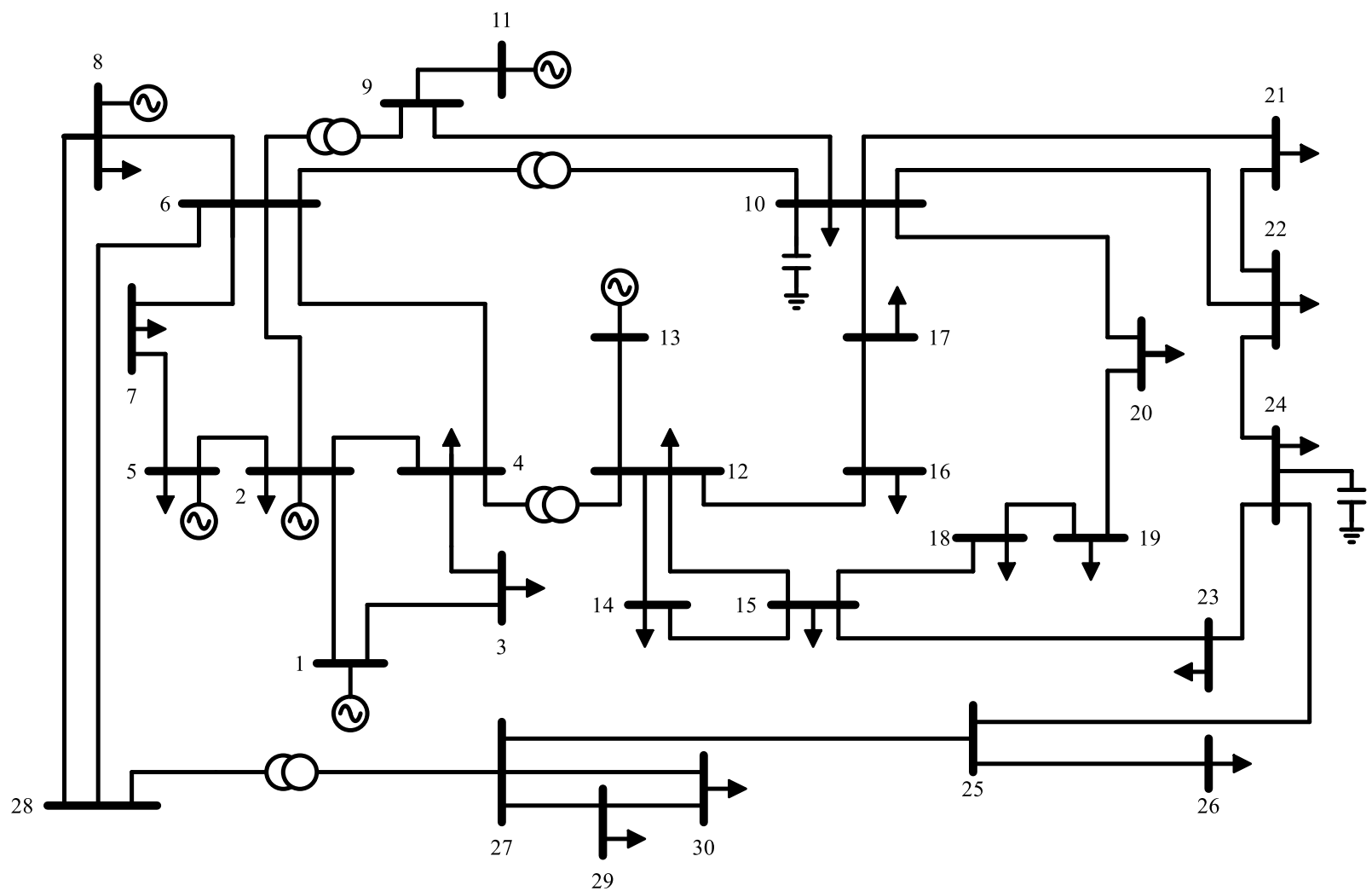

Figure A1. Single-line diagram of the IEEE 30-bus test system.

Table A1. System data of units with single-fuel coefficients considering valve-point effect and emission coefficients.

\begin{tabular}{|c|c|c|c|c|c|c|c|c|c|c|c|c|}
\hline Unit & $a_{i}$ & $b_{i}$ & $c_{i}$ & $d_{i}$ & $e_{i}$ & $\alpha_{i}$ & $\beta_{i}$ & $\gamma_{i}$ & $\xi_{i}$ & $\lambda_{i}$ & $\begin{array}{c}P_{\min } \\
(\mathbf{M W})\end{array}$ & $\begin{array}{c}P_{\max } \\
(\mathrm{MW})\end{array}$ \\
\hline $\mathrm{G}_{1}$ & 0.00375 & 2.00 & 0.0 & 18.00 & 0.037 & 0.04091 & -0.0005554 & 0.000006490 & 0.000200 & 0.02857 & 50 & 250 \\
\hline $\mathrm{G}_{2}$ & 0.01750 & 1.75 & 0.0 & 16.00 & 0.038 & 0.02543 & -0.0006047 & 0.000005638 & 0.000500 & 0.03333 & 20 & 80 \\
\hline $\mathrm{G}_{5}$ & 0.06250 & 1.00 & 0.0 & 14.00 & 0.040 & 0.04258 & -0.0005094 & 0.000004586 & 0.000001 & 0.08000 & 15 & 50 \\
\hline $\mathrm{G}_{8}$ & 0.00830 & 3.25 & 0.0 & 12.00 & 0.045 & 0.05326 & -0.0003550 & 0.000003380 & 0.002000 & 0.02000 & 10 & 35 \\
\hline $\mathrm{G}_{11}$ & 0.02500 & 3.00 & 0.0 & 13.00 & 0.042 & 0.04258 & -0.0005094 & 0.000004586 & 0.000001 & 0.08000 & 10 & 30 \\
\hline $\mathrm{G}_{13}$ & 0.02500 & 3.00 & 0.0 & 13.50 & 0.041 & 0.06131 & -0.0005555 & 0.000005151 & 0.000010 & 0.06667 & 12 & 40 \\
\hline
\end{tabular}

Table A2. Multi-fuel quadratic cost coefficients and output power boundary of units.

\begin{tabular}{cccccc}
\hline $\begin{array}{c}\text { Unit } \\
\text { Number }\end{array}$ & $\boldsymbol{a}_{\boldsymbol{i}}$ & $\boldsymbol{b}_{\boldsymbol{i}}$ & $\boldsymbol{c}_{\boldsymbol{i}}$ & $\mathbf{P}_{\min }(\mathbf{M W})$ & $\mathbf{P}_{\max }(\mathbf{M W})$ \\
\hline \multirow{2}{*}{$\mathrm{G}_{1}$} & 0.0050 & 0.70 & 55.0 & 50 & 140 \\
& 0.0075 & 1.05 & 82.5 & 140 & 200 \\
\hline \multirow{2}{*}{$\mathrm{G}_{2}$} & 0.0100 & 0.30 & 40.0 & 20 & 55 \\
& 0.0200 & 0.60 & 80.0 & 55 & 80 \\
\hline
\end{tabular}


Table A3. Prohibited operating zones of units.

\begin{tabular}{ccc}
\hline Units & Zone 1 & Zone 2 \\
\hline$G_{1}$ & {$[55-66]$} & {$[80-120]$} \\
$G_{2}$ & {$[21-24]$} & {$[45-55]$} \\
$G_{5}$ & {$[30-36]$} & - \\
$G_{8}$ & {$[25-30]$} & - \\
$G_{11}$ & {$[25-28]$} & - \\
$G_{13}$ & {$[24-30]$} & - \\
\hline
\end{tabular}

Table A4. Branches data.

\begin{tabular}{|c|c|c|c|}
\hline From Bus & To Bus & R (p.u.) & $X$ (p.u.) \\
\hline 1 & 2 & 0.02 & 0.06 \\
\hline 1 & 3 & 0.05 & 0.19 \\
\hline 2 & 4 & 0.06 & 0.17 \\
\hline 3 & 4 & 0.01 & 0.04 \\
\hline 2 & 5 & 0.05 & 0.20 \\
\hline 2 & 6 & 0.06 & 0.18 \\
\hline 4 & 6 & 0.01 & 0.04 \\
\hline 5 & 7 & 0.05 & 0.12 \\
\hline 6 & 7 & 0.03 & 0.08 \\
\hline 6 & 8 & 0.01 & 0.04 \\
\hline 6 & 9 & 0.00 & 0.21 \\
\hline 6 & 10 & 0.00 & 0.56 \\
\hline 9 & 11 & 0.00 & 0.21 \\
\hline 9 & 10 & 0.00 & 0.11 \\
\hline 4 & 12 & 0.00 & 0.26 \\
\hline 12 & 13 & 0.00 & 0.14 \\
\hline 12 & 14 & 0.12 & 0.26 \\
\hline 12 & 15 & 0.07 & 0.13 \\
\hline 12 & 16 & 0.09 & 0.20 \\
\hline 14 & 15 & 0.22 & 0.20 \\
\hline 16 & 17 & 0.08 & 0.19 \\
\hline 15 & 18 & 0.11 & 0.22 \\
\hline 18 & 19 & 0.06 & 0.13 \\
\hline 19 & 20 & 0.03 & 0.07 \\
\hline 10 & 20 & 0.09 & 0.21 \\
\hline 10 & 17 & 0.03 & 0.08 \\
\hline 10 & 21 & 0.03 & 0.07 \\
\hline 10 & 22 & 0.07 & 0.15 \\
\hline 21 & 22 & 0.01 & 0.02 \\
\hline 15 & 23 & 0.10 & 0.20 \\
\hline 22 & 24 & 0.12 & 0.18 \\
\hline 23 & 24 & 0.13 & 0.27 \\
\hline 24 & 25 & 0.19 & 0.33 \\
\hline 25 & 26 & 0.25 & 0.38 \\
\hline 25 & 27 & 0.11 & 0.21 \\
\hline 28 & 27 & 0.00 & 0.40 \\
\hline 27 & 29 & 0.22 & 0.42 \\
\hline 27 & 30 & 0.32 & 0.60 \\
\hline 29 & 30 & 0.24 & 0.45 \\
\hline 8 & 28 & 0.06 & 0.20 \\
\hline 6 & 28 & 0.02 & 0.06 \\
\hline
\end{tabular}


Table A5. Loads data.

\begin{tabular}{ccc}
\hline Bus Number & Active Power (MW) & Reactive Power (MVAr) \\
\hline 2 & 21.7 & 12.7 \\
3 & 2.40 & 1.20 \\
4 & 7.60 & 1.60 \\
5 & 94.2 & 0.00 \\
6 & 0.00 & 0.00 \\
7 & 22.8 & 10.9 \\
8 & 30.0 & 30.0 \\
9 & 0.00 & 0.00 \\
10 & 5.80 & 2.00 \\
11 & 0.00 & 0.00 \\
12 & 11.2 & 7.50 \\
13 & 0.00 & 0.00 \\
14 & 6.20 & 1.60 \\
15 & 8.20 & 2.50 \\
16 & 3.50 & 1.80 \\
17 & 9.00 & 5.80 \\
18 & 3.20 & 0.90 \\
19 & 9.50 & 3.40 \\
20 & 2.20 & 0.70 \\
21 & 17.5 & 11.2 \\
22 & 0.00 & 0.00 \\
23 & 3.20 & 1.60 \\
24 & 8.70 & 6.70 \\
25 & 0.00 & 0.00 \\
26 & 3.50 & 2.30 \\
27 & 0.00 & 0.00 \\
28 & 0.00 & 0.00 \\
29 & 2.40 & 0.90 \\
30 & 10.6 & 1.90 \\
\hline & & \\
& 0.00 & \\
& &
\end{tabular}

\section{Appendix B Data for Test System 2 (IEEE 57-bus Test System)}

The single-line diagram of the IEEE 57-bus test system is portrayed in Figure A2, and its essential data regarding generators are tabulated in Table A6.

Table A6. System data of units with single-fuel coefficients considering valve-point effect and emission coefficients.

\begin{tabular}{|c|c|c|c|c|c|c|c|c|c|c|c|c|}
\hline Unit & $a_{i}$ & $b_{i}$ & $c_{i}$ & $d_{i}$ & $e_{i}$ & $\alpha_{i}$ & $\beta_{i}$ & $\gamma_{i}$ & $\xi_{i}$ & $\lambda_{i}$ & $\begin{array}{c}P_{\min } \\
(\mathbf{M W})\end{array}$ & $\begin{array}{c}P_{\max } \\
(\mathbf{M W})\end{array}$ \\
\hline $\mathrm{G}_{1}$ & 0.00375 & 2.00 & 0.0 & 18.00 & 0.037 & 0.04091 & -0.05554 & 0.06490 & 0.0002 & 0.286 & 0 & 575.88 \\
\hline $\mathrm{G}_{2}$ & 0.0175 & 1.75 & 0.0 & 16.00 & 0.038 & 0.02543 & -0.06047 & 0.05638 & 0.0005 & 0.333 & 0 & 100 \\
\hline $\mathrm{G}_{3}$ & 0.025 & 3.00 & 0.0 & 13.50 & 0.041 & 0.06131 & -0.05555 & 0.05151 & 0.0001 & 0.667 & 0 & 140 \\
\hline $\mathrm{G}_{6}$ & 0.00375 & 2.00 & 0.0 & 18.00 & 0.037 & 0.03491 & -0.05754 & 0.06390 & 0.0003 & 0.266 & 0 & 100 \\
\hline $\mathrm{G}_{8}$ & 0.0625 & 1.00 & 0.0 & 14.00 & 0.040 & 0.04258 & -0.05094 & 0.04586 & 0.0001 & 0.800 & 0 & 550 \\
\hline $\mathrm{G}_{9}$ & 0.0195 & 1.75 & 0.0 & 15.00 & 0.039 & 0.02754 & -0.05847 & 0.05238 & 0.0004 & 0.288 & 0 & 100 \\
\hline $\mathrm{G}_{12}$ & 0.00834 & 3.25 & 0.0 & 12.00 & 0.045 & 0.05326 & -0.03555 & 0.03380 & 0.0002 & 0.200 & 0 & 410 \\
\hline
\end{tabular}




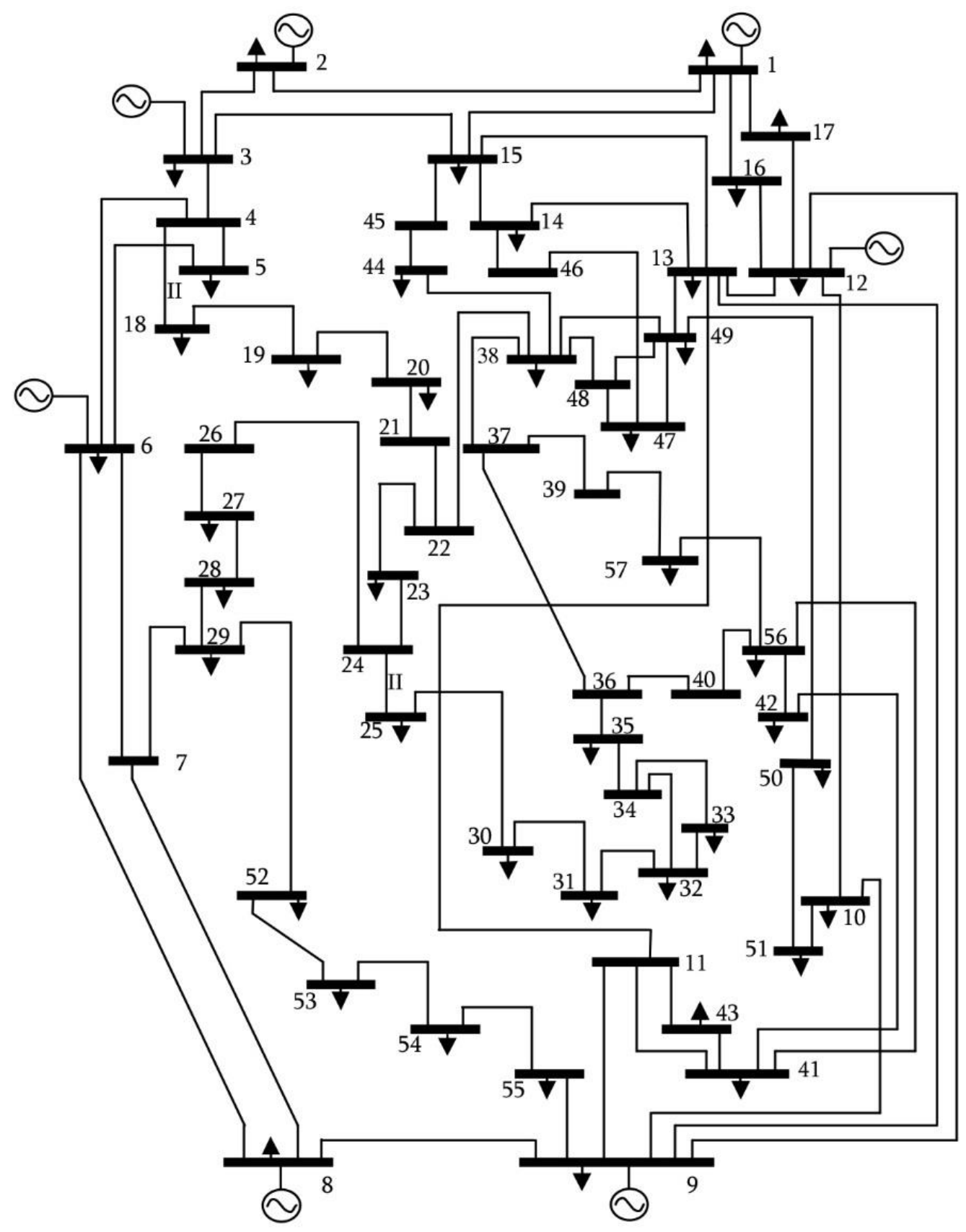

Figure A2. Single-line diagram of the IEEE 57-bus test system.

\section{Appendix C Data for Test System 3 (IEEE 118-bus Test System)}

The single-line diagram of the IEEE 118-bus test system is portrayed in Figure A3, and its essential data regarding emission coefficients of generators are tabulated in Table A7. 


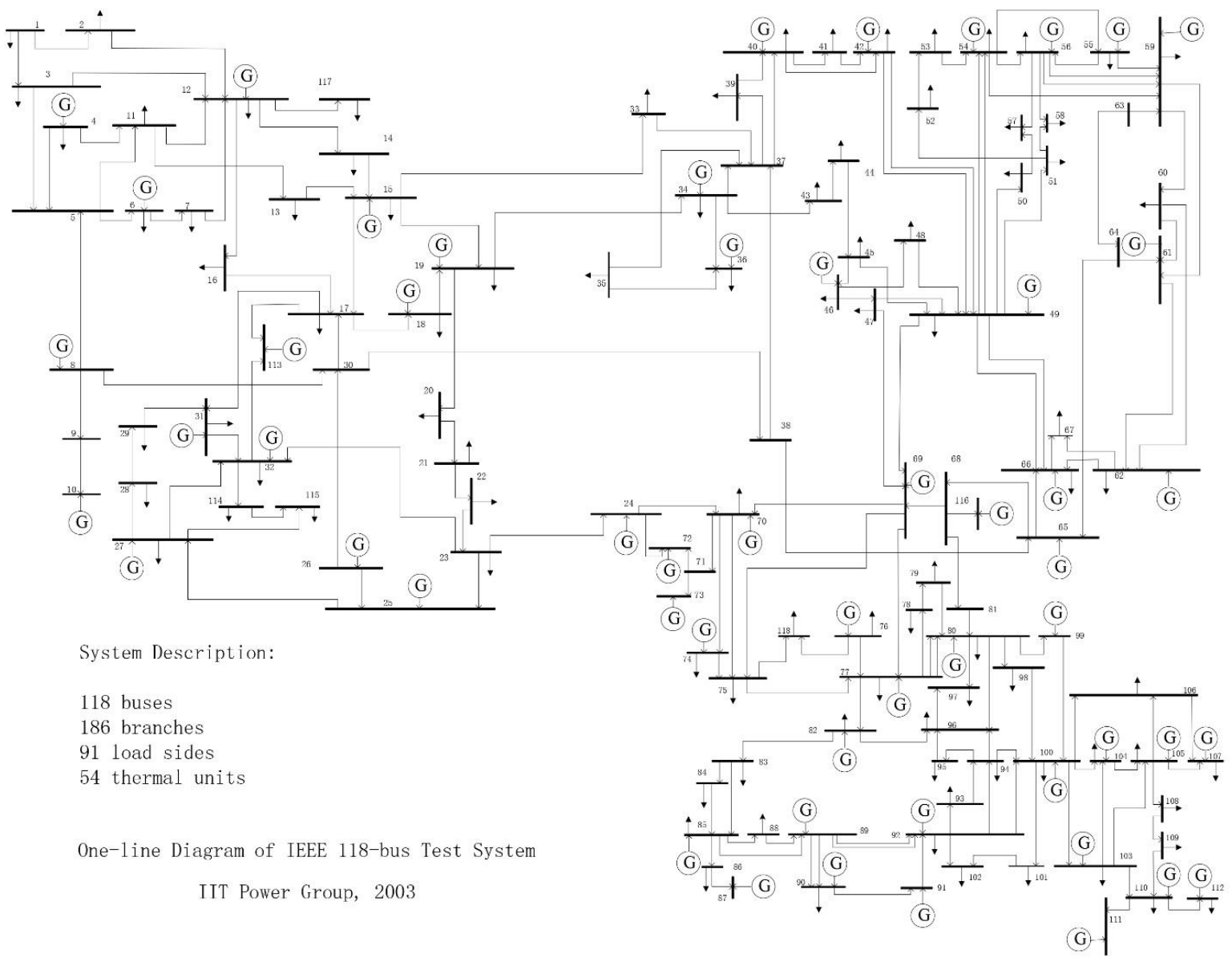

Figure A3. Single-line diagram of the IEEE 118-bus test system.

Table A7. Emission coefficients of units for linear model of emission function.

\begin{tabular}{cccc}
\hline Unit Number & $\sigma_{\boldsymbol{i}}$ & Unit Number & $\sigma_{\boldsymbol{i}}$ \\
\hline $\mathrm{G}_{4}$ & 0.0000 & $\mathrm{G}_{66}$ & 0.2000 \\
$\mathrm{G}_{6}$ & 0.0000 & $\mathrm{G}_{69}$ & 0.2000 \\
$\mathrm{G}_{8}$ & 0.0000 & $\mathrm{G}_{70}$ & 0.2000 \\
$\mathrm{G}_{10}$ & 0.0000 & $\mathrm{G}_{72}$ & 0.2000 \\
$\mathrm{G}_{12}$ & 0.0000 & $\mathrm{G}_{73}$ & 0.1500 \\
$\mathrm{G}_{15}$ & $\mathrm{G}_{74}$ & 0.1500 \\
$\mathrm{G}_{18}$ & 0.0000 & $\mathrm{G}_{76}$ & 0.1500 \\
$\mathrm{G}_{19}$ & 0.0000 & $\mathrm{G}_{77}$ & 0.1500 \\
$\mathrm{G}_{24}$ & 0.3500 & $\mathrm{G}_{80}$ & 0.1500 \\
$\mathrm{G}_{25}$ & 0.3500 & $\mathrm{G}_{82}$ & 0.0000 \\
$\mathrm{G}_{26}$ & 0.3500 & $\mathrm{G}_{85}$ & 0.0000 \\
$\mathrm{G}_{27}$ & 0.3500 & $\mathrm{G}_{87}$ & 0.0000 \\
$\mathrm{G}_{31}$ & $\mathrm{G}_{89}$ & 0.0000 \\
$\mathrm{G}_{32}$ & 0.0000 & $\mathrm{G}_{90}$ & 0.0000 \\
$\mathrm{G}_{34}$ & 0.0000 & $\mathrm{G}_{91}$ & 0.0000 \\
$\mathrm{G}_{36}$ & 0.0000 & $\mathrm{G}_{92}$ & 0.0000 \\
$\mathrm{G}_{40}$ & 0.3000 & $\mathrm{G}_{99}$ & 0.0000 \\
$\mathrm{G}_{42}$ & 0.3000 & $\mathrm{G}_{100}$ & 0.0000 \\
$\mathrm{G}_{46}$ & 0.3000 & $\mathrm{G}_{103}$ & 0.0000 \\
$\mathrm{G}_{49}$ & 0.3000 & $\mathrm{G}_{104}$ & 0.0000 \\
$\mathrm{G}_{54}$ & 0.0000 & $\mathrm{G}_{105}$ & 0.0000 \\
$\mathrm{G}_{55}$ & 0.0000 & $\mathrm{G}_{106}$ & 0.0000 \\
$\mathrm{G}_{56}$ & 0.2500 & $\mathrm{G}_{110}$ & 0.0000 \\
$\mathrm{G}_{59}$ & 0.2500 & $\mathrm{G}_{111}$ & 0.0000 \\
$\mathrm{G}_{61}$ & 0.2500 & $\mathrm{G}_{112}$ & 0.0000 \\
$\mathrm{G}_{62}$ & 0.2500 & $\mathrm{G}_{113}$ & 0.0000 \\
$\mathrm{G}_{65}$ & 0.0000 & $\mathrm{G}_{116}$ & 0.0000 \\
\hline
\end{tabular}




\section{References}

1. Naderi, E.; Pourakbari-Kasmaei, M.; Abdi, H. An efficient particle swarm optimization algorithm to solve optimal power flow problem integrated with FACTS devices. Appl. Soft Comput. 2019, 80, 243-262. [CrossRef]

2. Sun, D.I.; Ashley, B.; Brewer, B.; Hughes, A.; Tinney, W.F. Optimal power flow by Newton approach. IEEE Trans. Power Appar. Syst. 1984, 103, 2864-2875. [CrossRef]

3. Momoh, J.; El-Hawary, M.; Adapa, R. A review of selected optimal power flow literature to 1993. Part I and II. IEEE Trans. Power Syst. 1999, 14, 96-111. [CrossRef]

4. Burchet, R.C.; Happ, H.H.; Vierath, D.R. Quadratically convergent optimal power flow. IEEE Trans. Power Appar. Syst. 1984, 103, 3267-3276. [CrossRef]

5. Al-MuhaweshTareq, A.; Isa, S.Q. The established megawatt linear programming-based optimal power flow model applied to the real power 56-bus system in eastern province of Saudi Arabia. Energy 2008, 33, 12-21. [CrossRef]

6. Habibollahzadeh, H.; Luo, G.-X.; Semlyen, A. Hydrothermal optimal power flow based on combined linear and nonlinear programming methodology. IEEE Trans. Power Syst. 1989, 4, 530-537. [CrossRef]

7. Yan, X.; Quintana, V.H. Improving an interior point based OPF by dynamic adjustments of step sizes and tolerances. IEEE Trans. Power Syst. 1999, 14, 709-717.

8. Biskas, P.N.; Ziogos, N.P.; Tellidou, A.; Zoumas, C.E.; Bakirtzis, A.G.; Petridis, V. Comparison of two meta-heuristics with mathematical programming methods for the solution of OPF. IEE Proc. Gener. Transm. Distrib. 2006, 153, 16-24. [CrossRef]

9. AlRashidi, M.R.; El-Hawary, M.E. Hybrid particle swarm optimization approach for solving the discrete OPF problem considering the valve loading effects. IEEE Trans. Power Syst. 2007, 22, 2030-2038. [CrossRef]

10. Naderi, E.; Pourakbari-Kasmaei, M.; Lehtonen, M. Transmission expansion planning integrated with wind farms: A review, comparative study, and a novel profound search approach. Int. J. Electr. Power Energy Syst. 2020, 115, 105460. [CrossRef]

11. Azizivahed, A.; Arefi, A.; Naderi, E.; Narimani, H.; Fathi, M.; Narimani, M.R. An efficient hybrid approach to solve bi-objective multi-area dynamic economic emission dispatch problem. Electr. Power Compon. Syst. 2020, 48, 485-500. [CrossRef]

12. Naderi, E.; Asrari, A. Approaching optimal power flow from attacker's standpoint to launch false data injection cyberattack. In Proceedings of the IEEE Green Energy and Smart Systems Conference (IGESSC), Long Beach, CA, USA, 2-3 November 2020.

13. Niknam, T.; Narimani, M.R.; Jabbari, M. Dynamic optimal power flow using hybrid particle swarm optimization and simulated annealing. Eur. Trans. Electr. Power 2012, 23, 975-1001. [CrossRef]

14. Muhammad, Y.; Khan, R.; Raja, M.A.Z.; Ullah, F.; Chaudhary, N.I.; He, Y. Solution of optimal reactive power dispatch with FACTS devices: A survey. Energy Rep. 2020, 6, 2211-2229. [CrossRef]

15. Naderi, E.; Pourakbari-Kasmaei, M.; Cerna, F.V.; Lehtonen, M. A novel hybrid self-adaptive heuristic algorithm to handle singleand multi-objective optimal power flow problems. Int. J. Electr. Power Energy Syst. 2021, 125, 106492. [CrossRef]

16. Narimani, H.; Azizivahed, A.; Naderi, E.; Fathi, M.; Narimani, M.R. A practical approach for reliability-oriented multi-objective unit commitment problem. Appl. Soft Comput. 2019, 85, 105786. [CrossRef]

17. Belkacem, M.; Bouktir, T.; Srairi, K.; EL-Benbouzid, M. Dynamic strategy based fast decomposed GA coordinated with FACTS devices to enhance the optimal power flow. Energy Convers. Manag. 2010, 51, 1370-1380.

18. Reddy, S.S.; Bijwe, P.R.; Abhyankar, A.R. Faster evolutionary algorithm based optimal power flow using incremental variables. Int. J. Electr. Power Energy Syst. 2014, 54, 198-210. [CrossRef]

19. Kumari, M.S.; Maheswarapu, S. Enhanced genetic algorithm based computation technique for multi-objective optimal power flow solution. Int. J. Electr. Power Energy Syst. 2010, 32, 736-742. [CrossRef]

20. Lai, L.L.; Ma, J.T.; Yokoyama, R.; Zhao, M. Improved genetic algorithms for optimal power flow under both normal and contingent operation states. Int. J. Electr. Power Energy Syst. 1997, 19, 287-292. [CrossRef]

21. Kahourzade, S.; Mahmoudi, A.; Mokhlis, H.B. A comparative study of multi-objective optimal power flow based on particle swarm, evolutionary programming, and genetic algorithm. Electr. Energy 2015, 97, 1-12. [CrossRef]

22. Nithya, C.; Roselyn, J.P.; Devaraj, D.; Dash, S.S. Voltage stability constrained optimal power flow using non-dominated sorting genetic algorithm-II (NSGA-II). In Proceedings of the Third International Conference on Swarm, Evolutionary, and Memetic Computing, Bhubaneswar, India, 20-22 December 2012; pp. 793-801.

23. Mahdad, B.; Srairi, K.; Bouktir, T. Optimal power flow for large-scale power system with shunt FACTS using efficient parallel GA. Int. J. Electr. Power Energy Syst. 2010, 32, 507-517. [CrossRef]

24. Wirmond, V.E.; Fernandes, T.S.P.; Tortelli, O.L. TCPST allocation using optimal power flow and genetic algorithms. Int. J. Electr. Power Energy Syst. 2011, 33, 880-886. [CrossRef]

25. Szuvovivski, I.; Fernandes, T.S.P.; Aoki, A.R. Simultaneous allocation of capacitors and voltage regulators at distribution networks using genetic algorithms and optimal power flow. Int. J. Electr. Power Energy Syst. 2012, 40, 62-69. [CrossRef]

26. Kilc, U.; Ayan, K.; Arifoglu, U. Optimizing reactive power flow of HVDC systems using genetic algorithm. Int. J. Electr. Power Energy Syst. 2014, 55, 1-12. [CrossRef]

27. Kennedy, J.; Eberhart, R. Particle swarm optimization. In Proceedings of the ICNN'95 International Conference on Neural Networks, Perth, Australia, 27 November-1 December 1995; Volume 4, pp. 1942-1948.

28. Abido, M.A. Optimal power flow using particle swarm optimization. Int. J. Electr. Power Energy Syst. 2002, 24, 563-571. [CrossRef]

29. Singh, R.P.; Mukherjee, V.; Ghoshal, S.P. Particle swarm optimization with an aging leader and challengers algorithm for optimal power flow problem with FACTS devices. Int. J. Electr. Power Energy Syst. 2015, 64, 1185-1196. [CrossRef] 
30. Kumar, S.; Chaturvedi, D.K. Optimal power flow solution using fuzzy evolutionary and swarm optimization. Int. J. Electr. Power Energy Syst. 2013, 47, 416-423. [CrossRef]

31. Naderi, E.; Narimani, H.; Fathi, M.; Narimani, M.R. A novel fuzzy adaptive configuration of particle swarm optimization to solve large-scale optimal reactive power dispatch. Appl. Soft Comput. 2017, 53, 441-456. [CrossRef]

32. Esmin, A.A.A.; Lambert-Torres, G.; de Souza, A.C.Z. A hybrid particle swarm optimization applied to loss power minimization. IEEE Trans. Power Syst. 2005, 20, 859-866. [CrossRef]

33. Liang, R.-H.; Tsai, S.-R.; Chen, Y.-T.; Tseng, W.-T. Optimal power flow by a fuzzy based hybrid particle swarm optimization approach. Electr. Power Syst. Res. 2011, 81, 1466-1474. [CrossRef]

34. Chang, Y.-C.; Lee, T.-Y.; Chen, C.-L.; Jan, R.-M. Optimal power flow of a wind-thermal generation system. Int. J. Electr. Power Energy Syst. 2014, 55, 312-320. [CrossRef]

35. Vaisakh, K.; Srinivas, L.R.; Meah, K. Genetic evolving ant direction particle swarm optimization algorithm for optimal power flow with non-smooth cost functions and statistical analysis. Appl. Soft Comput. 2013, 13, 4579-4593. [CrossRef]

36. Niknam, T.; Narimani, M.R.; Aghaei, J.; Tabatabaei, S.; Nayeripour, M. Modified honey bee mating optimisation to solve dynamic optimal power flow considering generator constraints. IET Gener. Transm. Distrib. 2011, 5, 989-1002. [CrossRef]

37. Khorsandi, A.; Hosseinian, S.H.; Ghazanfari, A. Modified artificial bee colony algorithm based on fuzzy multi-objective technique for optimal power flow problem. Electr. Power Syst. Res. 2013, 95, 206-213. [CrossRef]

38. Adaryani, M.R.; Karami, A. Artificial bee colony algorithm for solving multi objective optimal power flow problem. Int. J. Electr. Power Energy Syst. 2013, 53, 219-230. [CrossRef]

39. Jadhav, H.T.; Roy, R. Stochastic optimal power flow incorporating offshore wind farm and electric vehicles. Int. J. Electr. Power Energy Syst. 2015, 69, 173-187. [CrossRef]

40. Roy, R.; Jadhav, H.T. Optimal power flow solution of power system incorporating stochastic wind power using Gbest guided artificial bee colony algorithm. Int. J. Electr. Power Energy Syst. 2015, 64, 562-578. [CrossRef]

41. Medina, M.A.; Das, S.; Coello, C.A.C.; Ramirez, J.M. Decomposition-based modern meta-heuristic algorithms for multi- objective optimal power flow: A comparative study. Eng. Appl. Artif. Intell. 2014, 32, 10-20. [CrossRef]

42. Armaghani, S.; Amjady, N.; Abedinia, O. Security constrained multi-period optimal power flow by a new enhanced artificial bee colony. Appl. Soft Comput. 2015, 37, 382-395. [CrossRef]

43. Chen, H.; Bo, M.L.; Zhu, Y. Multi-hive bee foraging algorithm for multi-objective optimal power flow considering the cost, loss, and emission. Int. J. Electr. Power Energy Syst. 2014, 60, 203-220. [CrossRef]

44. Ma, L.B.; Hu, K.Y.; Zhu, Y.L.; Chen, H.C. Improved multi-objective artificial bee colony algorithm for optimal power flow problem. J. Cent. South Univ. 2014, 21, 4220-4227. [CrossRef]

45. Ayan, K.; Kilic, U.; Barakli, B. Chaotic artificial bee colony algorithm based solution of security and transient stability constrained optimal power flow. Int. J. Electr. Power Energy Syst. 2015, 64, 136-147. [CrossRef]

46. Yuan, X.; Wang, P.; Yuan, Y.; Huang, Y.; Zhang, X. A new quantum inspired chaotic artificial bee colony algorithm for optimal power flow problem. Energy Convers. Manag. 2015, 100, 1-9. [CrossRef]

47. Ghasemi, A.; Valipour, K.; Tohidi, A. Multi-objective optimal reactive power dispatch using a new multi objective strategy. Int. J. Electr. Power Energy Syst. 2014, 57, 318-334. [CrossRef]

48. Ayan, K.; Kilic, U. Artificial bee colony algorithm solution for optimal reactive power flow. Appl. Soft Comput. 2012, 12, 1477-1482 [CrossRef]

49. Eusuff, M.M.; Lansey, K.E. Optimization of water distribution network design using the shuffled frog leaping algorithm. J. Water Resour. Plan. Manag. 2003, 129, 210-225. [CrossRef]

50. Niknam, T.; Narimani, M.R.; Jabbari, M.; Malekpour, A.R. A modified shuffle frog leaping algorithm for multi-objective optimal power flow. Energy 2011, 36, 6420-6432. [CrossRef]

51. Azizipanah-Abarghooee, R.; Narimani, M.R.; Bahmani-Firouzi, B.; Niknam, T. Modified shuffled frog leaping algorithm for multi-objective optimal power flow with FACTS devices. J. Intell. Fuzzy Syst. 2014, 26, 681-692. [CrossRef]

52. Sedighizadeh, M.; Sarvi, M.; Naderi, E. Multi-objective optimal power flow with FACTs devices using shuffled frog leaping algorithm. Int. Rev. Electr. Eng. 2011, 6, 1794-1801.

53. Khorsandi, A.; Alimardani, A.; Vahidi, B.; Hosseinian, S.H. Hybrid shuffled frog leaping algorithm and Nelder-Mead simplex search for optimal reactive power dispatch. IET Gener. Transm. Distrib. 2011, 5, 249-256. [CrossRef]

54. Yang, X.S. Nature-Inspired Meta-Heuristic Algorithms, 1st ed.; Luniver Press: Frome, UK, 2010.

55. Rao, B.V.; Kumar, G.V.N. Optimal power flow by BAT search algorithm for generation reallocation with unified power flow controller. Int. J. Electr. Power Energy Syst. 2015, 68, 81-88.

56. Kumar, Y.N.V.; Sivanagaraju, S.; Suresh, C.V. Security constrained economic load dispatch in the presence of interline power flow controller using modified BAT algorithm. Ain Shams Eng. J. 2016, 7, 1029-1043. [CrossRef]

57. Liang, R.-H.; Wang, J.-C.; Chen, Y.-T.; Tseng, W.-T. An enhanced firefly algorithm to multi-objective optimal active/reactive power dispatch with uncertainties consideration. Int. J. Electr. Power Energy Syst. 2015, 64, 1088-1097. [CrossRef]

58. Manoranjitham, G.E.; Alagarsamy, S. Application of firefly algorithm on optimal power flow control incorporating simplified impedance UPFC model. Int. J. Electr. Power Energy Syst. 2015, 71, 358-363. [CrossRef]

59. Mahdad, B.; Srairi, K. Security optimal power flow considering loading margin stability using hybrid FFA-PS assisted with brainstorming rules. Appl. Soft Comput. 2015, 35, 291-309. [CrossRef] 
60. Rashedi, E.; Nezamabadi-pour, H.; Saryazdi, S. GSA: A gravitational search algorithm. Inf. Sci. 2009, 179, 2232-2248. [CrossRef]

61. Bhowmik, A.R.; Chakraborty, A.K. Solution of optimal power flow using non dominated sorting multi-objective opposition based gravitational search algorithm. Int. J. Electr. Power Energy Syst. 2015, 64, 1237-1250. [CrossRef]

62. Duman, S.; Guvenc, U.; Sonmez, Y.; Yorukeren, N. Optimal power flow using gravitational search algorithm. Energy Convers. Manag. 2012, 59, 86-95. [CrossRef]

63. Bhattacharya, A.; Roy, P.K. Solution of multi-objective optimal power flow using gravitational search algorithm. IET Gener. Transm. Distrib. 2012, 6, 751-763. [CrossRef]

64. Radosavljevic, J.; Jevtic, M.; Arsic, N.; Klimenta, D. Optimal power flow for distribution networks using gravitational search algorithm. Electr. Energy 2014, 96, 335-345. [CrossRef]

65. Shaw, B.; Mukherjee, V.; Ghoshal, S.P. Solution of optimal power flow with FACTS devices using opposition-based gravitational search algorithm. Adv. Intell. Systrm Comput. 2015, 8947, 661-673.

66. Atashpaz-Gargari, E.; Lucas, C. Imperialist competitive algorithm: An algorithm for optimization inspired by imperialistic competition. In Proceedings of the 2007 IEEE Congress on Evolutionary Computation, Singapore, 25-28 September 2007; pp. 4661-4667.

67. Ardeshiri-Lajimi, R.; Amraee, T. A two stage model for rotor angle transient stability constrained optimal power flow. Int. J. Electr. Power Energy Syst. 2016, 76, 82-89. [CrossRef]

68. Ghasemi, M.; Ghavidel, S.; Rahmani, S.; Roosta, A.; Falah, H. A novel hybrid algorithm of imperialist competitive algorithm and Teaching learning algorithm for optimal power flow problem with non-Smooth cost functions. Eng. Appl. Artif. Intell. 2014, 29, 54-69. [CrossRef]

69. Ghasemi, M.; Ghavidel, S.; Ghanbarian, M.M.; Gharibzadeh, M.; Azizi-Vahed, A. Multi-objective optimal power flow considering the cost, emission, voltage deviation and power losses using multi-objective modified imperialist competitive algorithm. Energy 2014, 78, 276-289. [CrossRef]

70. Ghasemi, M.; Ghavidel, S.; Ghanbarian, M.M.; Massrur, H.R.; Gharibzadeh, M. Application of imperialist competitive algorithm with its modified techniques for multi-objective optimal power flow problem: A comparative study. Inf. Sci. 2014, 281, 225-247. [CrossRef]

71. Yang, X.S.; Deb, S. Cuckoo search via levy flights. In Proceedings of the 2009 World Congress on Nature and Biologically Inspired Computing, Bhubaneswar, India, 8-10 February 2009; pp. 210-214.

72. Anh, T.N.L.; Vo, D.N.; Ongsakul, W.; Vasant, P. Cuckoo optimization algorithm for optimal power flow. In Proceedings of the 18th Asia Pacific Symposium on Intelligent and Evolutionary Systems, Singapore, 10-12 November 2014; pp. 479-493.

73. Reddy, M.B.; Obulesh, Y.P.; Raju, S.S.; Suresh, C.V. Optimal power flow analysis by using hybrid cuckoo search algorithm. Int. J. Eng. Res. Technol. 2014, 3, 1514-1519.

74. Lenin, K.; Reddy, B.R.; Kalavathi, M.S. Improved cuckoo search algorithm for solving optimal reactive power dispatch problem. Int. J. Res. Electron. Commun. Technol. 2014, 1, 20-24.

75. Passino, K.M. Biomimicry of bacterial foraging for distributed optimization and control. IEEE Control. Syst. $2002,22,52-67$.

76. Tang, W.J.; Li, M.S.; Wu, Q.H.; Saunders, J.R. Bacterial foraging algorithm for optimal power flow in dynamic environments. IEEE Trans. Circuits Syst. 2008, 55, 2433-2442. [CrossRef]

77. Panda, A.; Tripathy, M. Optimal power flow solution of wind integrated power system using modified bacteria foraging algorithm. Int. J. Electr. Power Energy Syst. 2014, 54, 306-314. [CrossRef]

78. Edward, J.B.; Rajasekar, N.; Sathiyasekar, K.; Senthilnathan, N.; Sarjila, R. An enhanced bacterial foraging algorithm approach for optimal power flow problem including FACTS devices considering system loadability. ISA Trans. 2013, 52, 622-628. [CrossRef] [PubMed]

79. Tripathy, M.; Mishra, S. Bacteria foraging-based solution to optimize both real power loss and voltage stability limit. IEEE Trans. Power Syst. 2007, 22, 240-248. [CrossRef]

80. Panda, A.; Tripathy, M. Security constrained optimal power flow solution of wind-thermal generation system using modified bacteria foraging algorithm. Energy 2015, 93, 816-827. [CrossRef]

81. Geem, Z.W.; Kim, J.H. A new heuristic optimization algorithm: Harmony search. Simulation 2001, 76, 60-68. [CrossRef]

82. Sinsuphan, N.; Leeton, U.; Kulworawanichpong, T. Optimal power flow solution using improved harmony search method. Appl. Soft Comput. 2013, 13, 2364-2374. [CrossRef]

83. Sivasubramani, S.; Swarup, K.S. Multi-objective harmony search algorithm for optimal power flow problem. Int. J. Electr. Power Energy Syst. 2011, 33, 745-752. [CrossRef]

84. Khazali, A.H.; Kalantar, M. Optimal reactive power dispatch based on harmony search algorithm. Int. J. Electr. Power Energy Syst. 2011, 33, 684-692. [CrossRef]

85. Dorigo, M. Optimization, Learning and Natural Algorithms. Ph.D. Thesis, Politecnico de Milano, Milan, Italy, 1992.

86. Soares, J.; Sousa, T.; Vale, Z.A.; Morais, H.; Faria, P. Ant colony search algorithm for the optimal power flow problem. In Proceedings of the 2011 IEEE Power and Energy Society General Meeting, Detroit, MI, USA, 24-28 July 2011; pp. 1-8.

87. Metropolis, N.; Rosenbluth, A.W.; Rosenbluth, M.N.; Teller, M.; Teller, E. Equation of state calculations by very fast computing machines. J. Chem. Phys. 1953, 21, 1087-1092. [CrossRef]

88. Roa-Sepulveda, C.A.; Pavez-Lazo, B.J. A solution to the optimal power flow using simulated annealing. Int. J. Electr. Power Energy Syst. 2005, 25, 47-57. [CrossRef] 
89. Zhang, J.; Liu, K.; Tan, Y.; He, X. Random black hole particle swarm optimization and its application. In Proceedings of the IEEE International Conference Neural Networks \& Signal Processing, Zhenjiang, China, 8-10 June 2008; pp. 359-365.

90. Hatamlou, A. Black hole: A new heuristic optimization approach for data clustering. Inf. Sci. 2013, 222, 175-184. [CrossRef]

91. Azizipanah-Abarghooee, R.; Niknam, T.; Malekpour, M.; Bavafa, F.; Kaji, M. Optimal power flow based TU/CHP/PV/WPP coordination in view of wind speed, solar irradiance and load correlations. Energy Convers. Manag. 2015, 96, 131-145. [CrossRef]

92. Bouchekara, H.R.E.H. Optimal power flow using black-hole-based optimization approach. Appl. Soft Comput. 2014, 24, 879-888. [CrossRef]

93. Rao, R.; Savsani, V.; Vakharia, D. Teaching-learning-based optimization: A novel method for constrained mechanical design optimization problems. Comput. Aided Des. 2011, 43, 303-315. [CrossRef]

94. Niknam, T.; Azizipanah-Abarghooee, R.; Narimani, M.R. A new multi objective optimization approach based on TLBO for location of automatic voltage regulators in distribution systems. Eng. Appl. Artif. Intell. 2012, 25, 1577-1588. [CrossRef]

95. Ghasemi, M.; Ghavidel, S.; Gitizadeh, M.; Akbari, E. An improved teaching-learning-based optimization algorithm using Levy mutation strategy for non-smooth optimal power flow. Int. J. Electr. Power Energy Syst. 2015, 65, 375-384. [CrossRef]

96. Shabanpour-Haghighi, A.; Seifi, A.R.; Niknam, T. A modified teaching-learning based optimization for multi-objective optimal power flow problem. Energy Convers. Manag. 2014, 77, 597-607. [CrossRef]

97. Mandal, B.; Roy, P.K. Multi-objective optimal power flow using quasi-oppositional teaching learning based optimization. Appl. Soft Comput. 2014, 21, 590-606. [CrossRef]

98. Bouchekara, H.R.E.H.; Abido, M.A.; Boucherma, M. Optimal power flow using Teaching-Learning-Based Optimization technique. Electr. Power Syst. Res. 2014, 114, 49-59. [CrossRef]

99. Nayak, M.R.; Nayak, C.K.; Rout, P.K. Application of multi-objective teaching-learning based optimization algorithm to optimal power flow problem. Procedia Technol. 2012, 6, 255-264. [CrossRef]

100. Manda, B.; Roy, P.K. Optimal reactive power dispatch using quasi-oppositional teaching learning based optimization. Int. J. Electr. Power Energy Syst. 2013, 53, 123-134. [CrossRef]

101. Honorio, L.M.; Leite da Silva, A.M.; Barbosa, D.A.; Delboni, L.F.N. Solving optimal power flow problems using a probabilistic $\alpha$-constrained evolutionary approach. IET Gener. Transm. Distrib. 2010, 4, 674-682. [CrossRef]

102. Hugang, X.; Haozhong, C.; Haiyu, L. Optimal reactive power flow incorporating static voltage stability based on multi-objective adaptive immune algorithm. Energy Convers. Manag. 2008, 49, 1175-1181.

103. Storn, R.; Price, K. A simple and efficient heuristic for global optimization over continuous spaces. J. Glob. Optim. 1997, 11, 341-359. [CrossRef]

104. Acharjee, P. Optimal power flow with UPFC using security constrained self-adaptive differential evolutionary algorithm for restructured power system. Int. J. Electr. Power Energy Syst. 2016, 76, 69-81. [CrossRef]

105. Sivasubramani, S.; Swarup, K.S. Multiagent based differential evolution approach to optimal power flow. Appl. Soft Comput. 2012, 12, 735-740. [CrossRef]

106. Amjady, N.; Sharifzadeh, H. Security constrained optimal power flow considering detailed generator model by a new robust differential evolution algorithm. Electr. Power Syst. Res. 2011, 81, 740-749. [CrossRef]

107. Babu, A.V.N.; Ramana, T.; Sivanagaraju, S. Analysis of optimal power flow problem based on two stage initialization algorithm. Int. J. Electr. Power Energy Syst. 2014, 55, 91-99. [CrossRef]

108. Basu, M. Multi-objective optimal power flow with FACTS devices. Energy Convers. Manag. 2011, 52, 903-910. [CrossRef]

109. Mahdad, B.; Srairi, K. Solving multi-objective optimal power flow problem considering wind-STATCOM using differential evolution. Energy 2013, 7, 75-89. [CrossRef]

110. Sivasubramani, S.; Swarup, K.S. Sequential quadratic programming based differential evolution algorithm for optimal power flow problem. IET Gener. Transm. Distrib. 2011, 5, 1149-1154. [CrossRef]

111. El Ela, A.A.A.; Abido, M.A.; Spea, S.R. Optimal power flow using differential evolution algorithm. Electr. Power Syst. Res. 2010, 80, 878-885. [CrossRef]

112. Cai, H.R.; Chung, C.Y.; Wong, K.P. Application of differential evolution algorithm for transient stability constrained optimal power flow. IEEE Trans. Power Syst. 2008, 23, 719-728. [CrossRef]

113. Sayah, S.; Zehar, K. Modified differential evolution algorithm for optimal power flow with non-smooth cost functions. Energy Convers. Manag. 2008, 49, 3036-3042. [CrossRef]

114. Basu, M. Optimal power flow with FACTS devices using differential evolution. Int. J. Electr. Power Energy Syst. 2008, 30, 150-156. [CrossRef]

115. Liang, C.H.; Chung, C.Y.; Wong, K.P.; Duan, X.Z. Parallel optimal reactive power flow based on cooperative co-evolutionary differential evolution and power system decomposition. IEEE Trans. Power Syst. 2007, 22, 249-257. [CrossRef]

116. Varadarajan, M.; Swarup, K.S. Differential evolutionary algorithm for optimal reactive power dispatch. Int. J. Electr. Power Energy Syst. 2008, 30, 435-441. [CrossRef]

117. Fogel, L.J. Intelligence through Simulated Evolution: Forty Years of Evolutionary Programming; John Wiley \& Sons: New York, NY, USA, 1999.

118. Shi, L.; Xu, G. Self-adaptive evolutionary programming and its application to multi-objective optimal operation of power systems. Electr. Power Syst. Res. 2001, 57, 181-187. [CrossRef] 
119. Shi, L.; Wang, C.; Yao, L.; Ni, Y.; Bazargan, M. Optimal power flow solution incorporating wind power. IEEE Syst. J. 2012, 6, 233-241. [CrossRef]

120. Sood, Y.R. Evolutionary programming based optimal power flow and its validation for deregulated power system analysis. Int. J. Electr. Power Energy Syst. 2007, 29, 65-75. [CrossRef]

121. Yuryevich, J.; Wong, K.P. Evolutionary programming based optimal power flow algorithm. IEEE Trans. Power Syst. 1999, 14, 1245-1250. [CrossRef]

122. Glover, F. Future paths for integer programming and links to artificial intelligence. Comput. Oper. Res. 1986, 13, 533-549. [CrossRef]

123. Abido, M.A. Optimal power flow using tabu search algorithm. Electr. Power Compon. Syst. 2002, 30, 469-483. [CrossRef]

124. He, S.; Wu, Q.H.; Saunders, J.R. A novel group search optimizer inspired by animal behavioral ecology. In Proceedings of the IEEE Congress on Evolutionary Computation 2006, Vancouver, BC, Canada, 16-21 July 2006; pp. 1272-1278.

125. Daryani, N.; Hagh, M.T.; Teimourzadeh, S. Adaptive group search optimization algorithm for multi-objectiveoptimal power flow problem. Appl. Soft Comput. 2016, 38, 1012-1024. [CrossRef]

126. Xia, S.W.; Zhou, B.; Chan, K.W.; Guo, Z.Z. An improved GSO method for discontinuous non-convex transient stability constrained optimal power flow with complex system model. Int. J. Electr. Power Energy Syst. 2015, 64, 483-492. [CrossRef]

127. Simon, D. Biogeography-based optimization. IEEE Trans. Evol. Comput. 2008, 12, 702-713. [CrossRef]

128. Kumar, A.R.; Premalatha, L. Optimal power flow for a deregulated power system using adaptive real coded biogeography-based optimization. Int. J. Electr. Power Energy Syst. 2015, 73, 393-399. [CrossRef]

129. Christy, A.A.; Ajay, P.; Raj, D.V. Adaptive biogeography based predator-prey optimization technique for optimal power flow. Int. J. Electr. Power Energy Syst. 2014, 62, 344-352. [CrossRef]

130. Roy, P.K.; Ghoshal, S.P.; Thakur, S.S. Biogeography based optimization for multi constraint optimal power flow with emission and non-smooth cost function. Expert Syst. Appl. 2010, 37, 8221-8228. [CrossRef]

131. Kaveh, A.; Talatahari, S. A novel heuristic optimization method: Charged system search. Acta Mech. 2010, 213, 267-289. [CrossRef]

132. Niknam, T.; Azizipanah-Abarghooee, R.; Narimani, M.R. Reserve constrained dynamic optimal power flow subject to valve-point effects, prohibited zones and multi-fuel constraints. Energy 2012, 47, 451-464. [CrossRef]

133. Mehrabian, A.R.; Lucas, C. A novel numerical optimization algorithm inspired from weed colonization. Ecol. Inf. 2006, 1, 355-366. [CrossRef]

134. Ghasemi, M.; Ghavidel, S.; Akbari, E.; Azizivahed, A. Solving non-linear, non-smooth and non-convex optimal power flow problems using chaotic invasive weed optimization algorithms based on chaos. Energy 2014, 73, 340-353. [CrossRef]

135. Civivioglu, P. Backtracking search optimization algorithm for numerical optimization problems. Appl. Math. Comput. 2013, 219, 8121-8144.

136. Kilic, U. Backtracking search algorithm-based optimal power flow with valve point effect and prohibited zones. Electr. Energy 2015, 97, 101-110. [CrossRef]

137. Cheng, M.Y.; Prayogo, D. Symbiotic organisms search: A new meta-heuristic optimization algorithm. Comput. Struct. 2014, 139, 98-112. [CrossRef]

138. Prasad, D.; Mukherjee, V. A novel symbiotic organisms search algorithm for optimal power flow of power system with FACTS devices. Eng. Sci. Technol. Int. J. 2015. [CrossRef]

139. Husseinzadeh-Kashan, A. League championship algorithm: A new algorithm for numerical function optimization. In Proceedings of the 2009 International Conference of Soft Computing and Pattern Recognition, Malacca, Malaysia, 4-7 December 2009; pp. 43-48.

140. Pourali, Z.; Aminnayeri, M. A novel discrete league championship algorithm for minimizing earliness/tardiness penalties with distinct due dates and batch delivery consideration. ICIC 2011, 1, 139-146.

141. Bouchekara, H.R.E.H.; Abido, M.A.; Chaib, A.E.; Mehasni, R. Optimal power flow using the league championship algorithm: A case study of the Algerian power system. Energy Convers. Manag. 2014, 87, 58-70. [CrossRef]

142. De Castro, L.N.; Von Zuben, F.J. Learning and optimization using the clonal selection principle. IEEE Trans. Evol. Comput. 2002, 6, 239-251. [CrossRef]

143. Rao, B.S.; Vaisakh, K. Multi-objective adaptive clonal selection algorithm for solving optimal power flow considering multi-type FACTS devices and load uncertainty. Appl. Soft Comput. 2014, 23, 286-297. [CrossRef]

144. Yang, X.S. Flower pollination algorithm for global optimization. In Unconventional Computation and Natural Computation, Lecture Notes in Computer Science; Springer: Berlin/Heidelberg, Germany, 2012; Volume 7445, pp. 240-249.

145. Regalado, J.A.; Barocio, E.E.; Cuevas, E. Optimal power flow solution using modified flower pollination algorithm. In Proceedings of the 2015 IEEE International Autumn Meeting on Power, Electronics and Computing, Ixtapa, Mexico, 4-6 November 2015.

146. Rao, R. Jaya: A simple and new optimization algorithm for solving constrained and unconstrained optimization problems. Int. J. Ind. Eng. 2016, 7, 19-34.

147. Warid, W.; Hizam, H.; Mariun, N.; Abdul-Wahab, N.I. Optimal power flow using the Jaya algorithm. Energies $2016,9,678$. [CrossRef]

148. Mirjalili, S.; Mirjalili, S.M.; Lewis, A. Grey wolf optimizer. Adv. Eng. Softw. 2014, 69, 46-61. [CrossRef]

149. Sultana, U.; Khariruddin, A.B.; Mokhtar, A.S.; Zareen, N.; Sultana, B. Grey wolf optimizer based placement and sizing of multiple distributed generation in the distribution system. Energy 2016, 111, 525-536. [CrossRef] 
150. El-Fergany, A.A.; Hasanien, H.M. Single and Multi-objective optimal power flow using grey wolf optimizer and differential evolution algorithms. Electr. Power Compon. Syst. 2015, 43, 1548-1559. [CrossRef]

151. Mirjalili, S.; Lewis, A. The whale optimization algorithm. Adv. Eng. Softw. 2016, 95, 51-67. [CrossRef]

152. Bhesdadiya, R.H.; Pamar, M.B.; Jangir, N. Optimal active and reactive power dispatch problem solution using whale optimization algorithm. Indian J. Sci. Technol. 2016, 9, 1-6. [CrossRef]

153. Mehdinejad, M.; Mohammadi-Ivatloo, B.; Dadashzadeh-bonab, R.; Zare, K. Solution of optimal reactive power dispatch of power systems using hybrid particle swarm optimization and imperialist competitive algorithms. Int. J. Electr. Power Energy Syst. 2016, 83, 104-116. [CrossRef]

154. Das, D.B.; Patvardhan, C. Useful multi-objective hybrid evolutionary approach to optimal power flow. IEE Proc. Gener. Transm. Distrib. 2003, 150, 275-282.

155. Ongsakul, W.; Bhasaputra, P. Optimal power flow with FACTS devices by hybrid TS-SA approach. Int. J. Electr. Power Energy Syst. 2002, 24, 851-857. [CrossRef]

156. Yan, W.; Lu, S.; Yu, D.C. A Novel Optimal Reactive Power Dispatch Method Based on an Improved Hybrid Evolutionary Programming Technique. IEEE Trans. Power Syst. 2004, 19, 913-918. [CrossRef]

157. Chung, C.Y.; Liang, C.H.; Wong, K.P.; Duan, X.Z. Hybrid algorithm of differential evolution and evolutionary programming for optimal reactive power flow. IET Gener. Transm. Distrib. 2010, 4, 84-93. [CrossRef]

158. Li, Y.; Wang, Y.; Li, B. A hybrid artificial bee colony assisted differential evolution algorithm for optimal reactive power flow. Int. J. Electr. Power Energy Syst. 2013, 52, 25-33. [CrossRef]

159. Huang, C.M.; Huang, Y.C. Hybrid optimisation method for optimal power flow using flexible AC transmission system devices. IET Gener. Transm. Distrib. 2014, 8, 2036-2045. [CrossRef]

160. Naderi, E.; Azizivahed, A.; Narimani, H.; Fathi, M.; Narimani, M.R. A comprehensive study of practical economic dispatch problems by a new hybrid evolutionary algorithm. Appl. Soft Comput. 2017, 61, 1186-1206. [CrossRef]

161. Azizivahed, A.; Naderi, E.; Narimani, H.; Fathi, M.; Narimani, M.R. A new bi-objective approach to energy management in distribution networks with energy storage systems. IEEE Trans. Sustain. Energy 2018, 9, 56-64. [CrossRef]

162. Narimani, H.; Razavi, S.E.; Azizivahed, A.; Naderi, E.; Fathi, M.; Ataei, M.H.; Narimani, M.R. A multi-objective framework for multi-area economic emission dispatch. Energy 2018, 154, 126-142. [CrossRef]

163. Azizivahed, A.; Narimani, H.; Naderi, E.; Fathi, M.; Narimani, M.R. A hybrid evolutionary algorithm for secure multi-objective distribution feeder reconfiguration. Energy 2017, 138, 355-373. [CrossRef]

164. Azizivahed, A.; Narimani, H.; Fathi, M.; Naderi, E.; Safarpour, H.R.; Narimani, M.R. Multi-objective dynamic distribution feeder reconfiguration in automated distribution systems. Energy 2018, 147, 896-914. [CrossRef]

165. Azizivahed, A.; Karandeh, R.; Cecchi, V.; Naderi, E.; Li, L.; Zhang, J. Multi-area dynamic economic dispatch considering water consumption minimization, wind generation, and energy storage systems. In Proceedings of the 2020 IEEE Power \& Energy Society Innovative Smart Grid Technologies (ISGT) Conference, Washington, DC, USA, 17-20 February 2020.

166. Aein, M.; Rashidinejad, M.; Firuz-Abad, M.F. Probabilistic optimal power flow in correlated hybrid wind-PV power systems: A review and a new approach. Renew. Sustain. Energy Rev. 2015, 41, 1437-1446. [CrossRef]

167. Behera, S.; Sahoo, S.; Pati, B.B. A review on optimization algorithms and application to wind energy integration to grid. Renew. Sustain. Energy Rev. 2015, 48, 214-227. [CrossRef]

168. Kishore, T.S.; Singh, S.K. Optimal economic planning of power transmission lines: A review. Renew. Sustain. Energy Rev. 2014, 39, 949-974. [CrossRef]

169. Prakash, P.; Khatod, D.K. Optimal sizing and siting techniques for distributed generation in distribution systems: A review. Renew. Sustain. Energy Rev. 2016, 57, 111-130. [CrossRef]

170. Hemmati, R.; Hooshmand, R.A.; Khodabakhshian, A. Market based transmission expansion and reactive power planning with consideration of wind and load uncertainties. Renew. Sustain. Energy Rev. 2014, 29, 1-10. [CrossRef]

171. Reddy, S.S.; Bijwe, P.R. Real time economic dispatch considering renewable energy resources. Renew. Energy 2015, 83, 1215-1226. [CrossRef]

172. Reddy, S.S.; Bijwe, P.R. Day-ahead and real time optimal power flow considering renewable energy resources. Int. J. Electr. Power Energy Syst. 2016, 82, 400-408. [CrossRef]

173. Reddy, S.S. Optimal power flow with renewable energy resources including storage. Electr. Eng. 2016, 99, 685-695. [CrossRef]

174. Shilaja, C.; Ravi, K. Optimal power flow considering intermittent wind power using particle swarm optimization. Int. J. Renew. Energy Res. 2016, 6, 504-509.

175. Makhloufi, S.; Mekhaldi, A.; Teguar, M.; Saheb-Koussa, D.; Djoudi, A. Optimal power flow solution including wind power generation into isolated Adrar power system using PSOGSA. Rev. Energies Renouvelables 2013, 16, 721-732.

176. Riffonneau, Y.; Bacha, S.; Barruel, F.; Ploix, S. Optimal power flow management for grid connected PV systems with batteries. IEEE Trans. Sustain. Energy 2011, 2, 309-320. [CrossRef]

177. Giraldo-Chavarriaga, J.S.; Castrillon-Largo, J.A.; Granada-Echeverri, M. Stochastic AC optimal power flow considering the probabilistic behavior of the wind, loads and line parameters. Ing. Investig. Tecnol. 2014, 15, 529-538.

178. Narimani, M.R.; Azizivahed, A.; Naderi, E. An efficient scenario-based stochastic energy management of distribution networks with distributed generation, PV module, and energy storage. arXiv 2019, arXiv:1910.07109. 
179. Amini, M.H.; Khazaei, J.; Khezrimotlagh, D.; Asrari, A. Bi-level adversary-operator cyberattack framework and algorithms for transmission networks in smart grids. In Optimization, Learning, and Control for Interdependent Complex Networks; Springer: Cham, Switzerland, 2020; pp. 183-203.

180. Pazouki, S.; Naderi, E.; Asrari, A. Interconnected energy hubs including DERs targeted by FDI cyberattacks. In Proceedings of the 2020 IEEE International Green and Sustainable Computing (IGSC) Conference, Pullman, WA, USA, 19-22 October 2020.

181. Khezrimotlagh, D.; Khazaei, J.; Asrari, A. MILP modeling of targeted false load data injection cyberattacks to overflow transmission lines in smart grids. In Proceedings of the 2019 North American Power Symposium (NAPS), Wichita, KS, USA, 13-15 October 2019.

182. Xiang, Y.; Wang, L.; Liu, N. Coordinated attacks on electric power systems in a cyber-physical environment. Electr. Power Syst. Res. 2017, 149, 156-168. [CrossRef]

183. Liu, Y.; Reiter, M.K.; Ning, P. False data injection attacks against state estimation in electric power grids. In Proceedings of the 16th ACM Conference on Computer and Communications Security, Chicago, IL, USA, 9-13 November 2009; pp. 21-32.

184. Liu, Y.; Ning, P.; Reiter, M.K. False data injection attacks against state estimation in electric power grids. ACM Trans. Inf. Syst. Secur. 2011, 14, 1-33. [CrossRef]

185. Xie, L.; Mo, Y.; Sinopoli, B. Integrity data attacks in power market operations. IEEE Trans. Smart Grid 2011, 2, 659-666. [CrossRef]

186. Liang, G.; Weller, S.R.; Luo, F.; Zhao, J.; Dong, Z.Y. Generalized FDIA-based cyber topology attack with application to the Australian electricity market trading mechanism. IEEE Trans. Smart Grid 2018, 9, 3820-3829. [CrossRef]

187. Esmalifalak, M.; Shi, G.; Han, Z.; Song, L. Bad data injection attack and defense in electricity market using game theory study. IEEE Trans. Smart Grid 2013, 4, 160-169. [CrossRef]

188. Che, L.; Liu, X.; Li, Z.; Wen, Y. False data injection attacks induced sequential outages in power systems. IEEE Trans. Power Syst. 2019, 34, 1513-1523. [CrossRef]

189. Liu, X.; Li, Z. Trilevel modeling of cyber attacks on transmission lines. IEEE Trans. Smart Grid 2017, 8, 720-729. [CrossRef]

190. Tan, Y.; Li, Y.; Cao, Y.; Shahidehpour, M. Cyber-attack on overloading multiple lines: A bilevel mixed-integer linear programming model. IEEE Trans. Smart Grid 2018, 9, 1534-1536. [CrossRef]

191. Deng, R.; Liang, H. False data injection attacks with limited susceptance information and new countermeasures in smart grid. IEEE Trans. Ind. Inform. 2019, 15, 1619-1628. [CrossRef]

192. Asrari, A.; Ansari, M.; Khazaei, J.; Cecchi, V. Real-time blackout prevention in response to decentralized cyberattacks on a smart grid. In Proceedings of the 2020 IEEE Texas Power and Energy Conference (TPEC), College Station, TX, USA, 6-7 February 2020; pp. 1-5.

193. Li, Y.; Wang, Y.; Hu, S. Online generative adversary network based measurement recovery in false data injection attacks: A cyber-physical approach. IEEE Trans. Ind. Inform. 2020, 16, 2031-2043. [CrossRef]

194. Huang, H.; Kazerooni, M.; Hossain-McKenzie, S.; Etigowni, S.; Zonouz, S.; Davis, K. Fast generation redispatch techniques for automated remedial action schemes. In Proceedings of the 2019 IEEE International Conference on Intelligent System Application to Power Systems (ISAP), New Delhi, India, 10-14 December 2019; pp. 1-8.

195. Shayan, H.; Amraee, T. Network constrained unit commitment under cyber attacks driven overloads. IEEE Trans. Smart Grid 2019, 10, 6449-6460. [CrossRef]

196. Ajao, B.; Khaledian, P.; Johnson, B.K.; Chakhchoukh, Y. Implementation of remedial action scheme for transient stability index improvement of power system island. In Proceedings of the 2020 IEEE Innovative Smart Grid Technologies (ISGT) Conference, Washington, DC, USA, 17-20 February 2020; pp. 1-5.

197. Khaledian, P.; Johnson, B.K.; Hemati, S. Power grid security improvement by remedial action schemes using vulnerability assessment based on fault chains and power flow. In Proceedings of the IEEE International Conference on Probabilistic Methods Applied to Power Systems (PMAPS), Boise, ID, USA, 24-28 June 2018; pp. 1-6.

198. Wang, P.; Govindarasu, M. Multi intelligent agent based cyber attack resilient system protection and emergency control. In Proceedings of the 2016 IEEE Innovative Smart Grid Technologies Conference, Minneapolis, MN, USA, 6-9 September 2016; pp. 1-5.

199. Naderi, E.; Pazouki, S.; Asrari, A. A region-based framework for cyberattacks leading to undervoltage in smart distribution systems. In Proceedings of the 2021 IEEE Power and Energy Conference at Illinois (PECI), Urbana-Champaign, Urbana, IL, USA, 1-2 April 2021.

200. Naderi, E.; Asrari, A. Hardware-in-the-loop experimental validation for a lab-scale microgrid targeted by cyberattacks. In Proceedings of the 9th International Conference on Smart Grid, Setubal, Portugal, 29 June-1 July 2021.

201. Naderi, E.; Pazouki, S.; Asrari, A. A remedial action scheme against false data injection cyberattacks in smart transmission systems: Application of thyristor controlled series capacitor (TCSC). IEEE Trans. Ind. Inform. 2021. early access. [CrossRef] 